\title{
Effective Dynamics for Boson Stars
}

\author{
Jürg Fröhlich*, B. Lars G. Jonsson, Enno Lenzmann
}

May 17, 2006

\begin{abstract}
We study solutions close to solitary waves of the pseudo-relativistic Hartree equation describing boson stars under the influence of an external gravitational field. In particular, we analyze the long-time effective dynamics of such solutions. In essence, we establish a (long-time) stability result for solutions describing boson stars that move under the influence of an external gravitational field.
\end{abstract}

\section{Introduction}

In this paper, we study boson stars described as solutions of the pseudo-relativistic Hartree equation which, initially, are close to a solitary wave. The pseudo-relativistic Hartree equation is the nonlinear evolution equation

$$
\mathrm{i} \partial_{t} \psi=\left(\sqrt{-\Delta+m^{2}}-m\right) \psi+V \psi-\left(\frac{1}{|x|} *|\psi|^{2}\right) \psi
$$

where $\psi=\psi(x, t)$ is a complex wave field on space-time, $x \in \mathbb{R}^{3}$ is a point in space, and $t \in \mathbb{R}$ is time. Here the symbol $*$ denotes spatial convolution. The kinetic energy operator $\sqrt{-\Delta+m^{2}}-m$ is appropriate to describe relativistic quantum particles of mass $m>0$. This operator is defined by its symbol $\sqrt{k^{2}+m^{2}}-m$ in momentum space. The convolution kernel, $|x|^{-1}$, represents the Newtonian potential of gravitational 2-body interactions. We use units such that the speed of light and Planck's constant are equal to unity. By rescaling $\psi$ we may set Newton's gravitational constant times $m^{2}$ equal to unity.

Equation (1.1) describes a system of gravitating bosonic particles in a regime where effects of special relativity are important, because the particles have velocities close to the speed of light, but retardation effects and space-time curvature can be neglected. As recently shown in [7], equation (1.1) emerges as the correct evolution equation for the mean-field dynamics of many-body quantum systems modelling pseudo-relativistic boson stars. The external potential, $V=V(x)$, accounts for gravitational fields from

${ }^{*}$ Addresses are given at the end. 
other stars. $V$ is a smooth, bounded, slowly varying real function; (precise assumptions on $V$ are stated in Section 3).

Equation (1.1) admits some important conserved quantities. Namely, the mass of the system (proportional to the number of particles), and its energy. These quantities are given by ${ }^{1}$

$$
\mathcal{N}(\psi):=\frac{1}{2} \int_{\mathbb{R}^{3}}|\psi|^{2} \mathrm{~d} x
$$

and

$$
\mathcal{H}_{V}(\psi):=\frac{1}{2} \int_{\mathbb{R}^{3}}\left(\left|\left(-\Delta+m^{2}\right)^{1 / 4} \psi\right|^{2}-m|\psi|^{2}+V|\psi|^{2}\right) \mathrm{d} x-\frac{1}{4} \int_{\mathbb{R}^{3}}\left(\frac{1}{|x|} *|\psi|^{2}\right)|\psi|^{2} \mathrm{~d} x,
$$

respectively. The momentum, $\mathcal{P}$, also plays an important role. It is defined by

$$
\mathcal{P}(\psi):=\frac{-\mathrm{i}}{2} \int_{\mathbb{R}^{3}} \bar{\psi} \nabla \psi \mathrm{d} x
$$

and is conserved when the external potential $V$ is constant.

In [20] it was shown for equation (1.1) that initial data $\psi_{0} \in \mathrm{H}^{\frac{1}{2}}$ with $\mathcal{N}(\psi)<$ $N_{\mathrm{c}}$, where $N_{\mathrm{c}}>2 / \pi$ is a universal constant, lead to global-in-time solutions $\psi \in$ $\mathrm{C}^{0}\left([0, \infty) ; \mathrm{H}^{\frac{1}{2}}\left(\mathbb{R}^{3}\right)\right) \cap \mathrm{C}^{1}\left([0, \infty) ; \mathrm{H}^{-\frac{1}{2}}\right)$. Furthermore, if $V \equiv 0$ holds then we have solitary wave solutions of (1.1) given by

$$
\psi(x, t)=\mathrm{e}^{\mathrm{i} t \mu} \varphi_{v, \mu}(x-v t) .
$$

Here the parameter $v \in \mathbb{R}^{3}$ corresponds to the travelling velocity, and the function $\varphi_{v, \mu} \in \mathrm{H}^{\frac{1}{2}}$ is a minimizer of the functional

$$
\mathcal{E}_{v, 0}:=\mathcal{H}_{V \equiv 0}(\psi)-v \cdot \mathcal{P}(\psi),
$$

subject to the constraint $\mathcal{N}(\psi)=N$, with $N<N_{\mathrm{c}}(v)$, where $N_{\mathrm{c}}(v)<N_{\mathrm{c}}$ is a finite constant. The minimizers $\varphi_{v, \mu}$ are referred to as boosted ground states. They solve the Euler-Lagrange equation

$$
\left(\sqrt{-\Delta+m^{2}}-m\right) \varphi+\mu \varphi+\mathrm{i} v \cdot \nabla \varphi-\left(\frac{1}{|x|} *|\varphi|^{2}\right) \varphi=0
$$

where the frequency $\mu$ is a Lagrange multiplier for the constraint $\mathcal{N}(\psi)=N$. As shown in our companion paper [9], such boosted ground state exists for all $|v|<1$, i.e., the travelling velocity is below the speed of light, and the constant $N_{\mathrm{c}}(v)$ satisfies the bounds $(1-|v|) N_{\mathrm{c}} \leq N_{\mathrm{c}}(v) \leq N_{\mathrm{c}}(0) \equiv N_{\mathrm{c}}$. In addition, we remark that the ground states, $\varphi_{v, \mu}$, decay exponentially, with rate $\delta=\delta(\mu, v)$. Further properties of $\varphi_{v, \mu}$ are established in Section 4; see Proposition 4.3.

The main goal of the present paper is to provide a detailed description of solutions of (1.1), initially close to a manifold of boosted ground states, over a long interval of

\footnotetext{
${ }^{1}$ Note that in $[9]$ we used $\mathcal{N}(\psi)=\int|\psi|^{2} \mathrm{~d} x$.
} 
time. In this study, two length scales will play a crucial role. The first one is determined by the external potential and is given by

$$
\ell_{\exp }:=\|\nabla V\|_{\infty}^{-1} .
$$

The second length scale is inferred from the requirement that the initial condition, $\psi_{0}$, of (1.1) be close to a ground state, $\varphi_{v, \mu}$. We will project, see Section 6 , such an initial condition to a point on a manifold of boosted ground states. All ground states are exponentially localized with rate $\delta=\delta(\mu, v)$. The projection singles out one ground state, with an associated length scale given by its exponential decay rate. Thus, the second length scale is defined by

$$
\ell_{\mathrm{sol}}:=\delta^{-1}
$$

In the regime where

$$
\varepsilon:=\frac{\ell_{\mathrm{sol}}}{\ell_{\exp }} \ll 1
$$

we expect that solutions of (1.1) with initial condition close to a ground state $\varphi_{v, \mu}$ behave like relativistic point particles.

We now sketch our Main Result. Let $\left(y^{(0)}, v^{(0)}, \vartheta^{(0)}, \mu^{(0)}\right)$ be a point in $\mathbb{R}^{3} \times \mathbb{R}^{3} \times$ $[0,2 \pi) \times \mathbb{R}_{+}$, with $|v|^{(0)} \leq r<1$ for some small $r>0$ and $\mu^{(0)} \in I \subset \mathbb{R}_{+}$, where $I$ is some open interval. We consider an initial condition, $\psi_{0} \in \tilde{\Gamma}$, such that

$$
\left\|\psi_{0}-\mathrm{e}^{\mathrm{i} \vartheta^{(0)}} \varphi_{v^{(0)}, \mu^{(0)}}\left(\cdot-y^{(0)}\right)\right\|_{\tilde{\Gamma}} \leq \varepsilon
$$

where $\tilde{\Gamma} \subset \mathrm{H}^{\frac{1}{2}}$ is a weighted Sobolev space with norm $\|\cdot\|_{\tilde{\Gamma}}$. We then show that the solution of (1.1) with initial condition $\psi_{0}$ remains close to a ground state, for times of order $\varepsilon^{-1}$. More explicitly, we prove that

$$
\psi(x, t)=\mathrm{e}^{\mathrm{i} \vartheta(t)}\left(\varphi_{v(t), \mu(t)}(x-y(t))+\xi(x-y(t), t)\right),
$$

with $\|\xi\|_{\tilde{\Gamma}} \leq C \varepsilon$, for times $0 \leq t \leq C \varepsilon^{-1}$. Here the time-dependent functions $(y, v, \vartheta, \mu)$ satisfy the Equations of Motion,

$$
\dot{y}=v+\mathcal{O}\left(\varepsilon^{2}\right), \quad \gamma(v, \mu) \dot{v}=-\nabla V(y)+\mathcal{O}\left(\varepsilon^{2}\right),
$$

where the factor $\gamma(v, \mu)$ is a relativistic correction, and

$$
\frac{\mathrm{d}}{\mathrm{d} t} \mathcal{N}\left(\boldsymbol{\varphi}_{v, \mu}\right)=\mathcal{O}\left(\varepsilon^{2}\right), \quad \dot{\vartheta}=V(y)-\mu+\mathcal{O}\left(\varepsilon^{2}\right)
$$

These results yield a fairly detailed description of the solution, $\psi(x, t)$, up to times of order $\varepsilon^{-1}$. For a precise statement of our main result, see Theorem 3.1 and its hypotheses in Section 3 below.

We remark that similar results for the Nonlinear Schrödinger Equation (NLS) can be found in $[13,8,16]$, and, for the Korteweg-de Vries equation, in [4, 3].

Next, we review some previous results for systems of gravitating relativistic bosons. One of the first studies of self-gravitating scalar bosons can be found in [27]. Important 
properties of a Hamiltonian describing a relativistic particle in an external potential proportional to $|x|^{-1}$ are proven in [14]; see also [34]. Bosonic matter is analyzed in [26]; and bosonic black holes are discussed in [33]. In these papers, it is argued that the Chandrasekhar limit for boson stars (with $m \approx 1-50 \mathrm{GeV}$ ) is approximately the mass of a mountain. Moreover, the intuitive scaling ideas used in $[26,33]$ are turned into rigorous statements in [22]. There, it is conjectured that the ground-state energy of $n$ bosonic particles with relativistic kinetic energy is given by the minimum of the pseudo-relativistic Hartree energy functional, $\mathcal{H}_{V \equiv 0}$, in the 'mean-field limit'. This has subsequently been shown in [23], where it is also proven that there exist minimizers, $\varphi_{\mu}$, for $\mathcal{H}_{V \equiv 0}(\psi)$ subject to the constraint $\mathcal{N}(\psi)=N<N_{\mathrm{c}}$, and that these minimizers can be chosen to

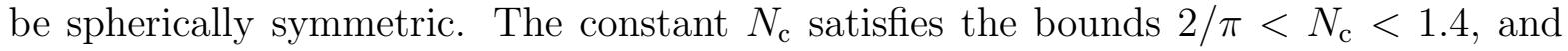
is interpreted as the critical mass for stability of a boson star; (bosonic Chandrasekhar limit mass).

A recent review paper on the mean-field limit of quantum Bose gases is paper [11], which contains rigorous results and a survey of open problems for Bose gases. It is shown in [20] that the initial value-problem for equation (1.1), is locally well-posed, satisfies a blow-up alternative and has global solutions for initial conditions $\psi_{0} \in \mathrm{H}^{\frac{1}{2}}\left(\mathbb{R}^{3}\right)$ with $\mathcal{N}\left(\psi_{0}\right)<N_{\mathrm{c}}$. The mean-field limit for systems of gravitating relativistic bosons is discussed in [7]. The existence of blow-up solutions of (1.1) with spherically symmetric initial conditions is shown in [12], using a virial-type argument. This blow-up result is indicative of "gravitational collapse" of Boson stars with mass beyond the (boson) Chandrasekhar limit. In a companion paper, [9], we show existence of boosted ground states $\varphi_{v, \mu}$, see (1.5), i.e., of minimizers of the functional $\mathcal{E}_{v, 0}(\psi)$, subject to the constraint $\mathcal{N}(\psi)=N<N_{\mathrm{c}}(v)$. Here $\mathcal{E}_{v, 0}$ is the functional defined in (1.6), above. We also prove exponential decay of these ground states and orbital stability of solutions of (1.1) with vanishing external potential. In paper [10], we present numerical evidence for the unproven (kernel) assumption used in this paper.

Equations (1.13), (1.14) can be seen as modulation equations. For previous work on modulation equations, see $[18,24,19,35,30,1,13,31,2,8,32,25,4,16]$.

The organization of our paper is as follows. In Section 2, we rephrase equation (1.1) as a Hamiltonian evolution equation and discuss its Hamiltonian structure. We also state a fundamental assumption. In Section 3, we state our main theorem and sketch its proof. Sections 4-10 contain numerous auxiliary results used in the proof of our main theorem. The main theorem is proven in Sect. 11. The appendices contain proofs of spectral properties and positivity of a certain linear operator, as well as the proof of Corollary 6.6.

Notation. The space of measurable functions, $f$, with $|f|^{p}$ integrable, is denoted by $\mathrm{L}^{p}$, and its norm by $\|\cdot\|_{p}$. For $p=2$, this space is the Hilbert space of square-integrable functions. The space of $n$ times continuously differentiable functions is denoted by $\mathrm{C}^{n}$. The usual inhomogeneous Sobolev space is denoted by $\mathrm{H}^{s}$, its norm by $\|\cdot\|_{\mathrm{H}^{s}}$. In particular,

$$
\|u\|_{\mathrm{H}^{\frac{1}{2}}}:=\left\|(1-\Delta)^{1 / 4} u\right\|_{2}
$$


for $u \in \mathrm{H}^{\frac{1}{2}}$. We define the weighted norm $\|\cdot\|_{\tilde{\Gamma}}$ by

$$
\|u\|_{\tilde{\Gamma}}^{2}:=\|u\|_{\mathrm{H}^{\frac{1}{2}}}^{2}+\varepsilon\left\||x|^{1 / 2} u\right\|_{2}^{2},
$$

for $\varepsilon>0$ given as in (1.10). We also use the notation $d_{t}:=\frac{\mathrm{d}}{\mathrm{d} t}$.

\section{The Hamiltonian Nature of Equation (1.1)}

Equation (1.1) is a Hamiltonian evolution equation on an infinite-dimensional phase space, $\Gamma$. In this paper we make extensive use of this fact and of the symplectic structure of the phase space. We therefore begin with a brief review of some basic notions in Hamiltonian dynamics.

The phase space, $\Gamma$, where (1.1) is well defined, for bounded $V$, is the complex Sobolev space $\mathrm{H}^{\frac{1}{2}}\left(\mathbb{R}^{3}, \mathbb{C}\right)$. A point in the phase space is identified with a complex-valued function $\psi \in \mathrm{H}^{\frac{1}{2}}\left(\mathbb{R}^{3}, \mathbb{C}\right)$. The decomposition of $\psi$ into real and imaginary parts,

$$
\psi=\psi^{(1)}+\mathrm{i} \psi^{(2)}
$$

where $\psi^{(1)}$ and $\psi^{(2)}$ are real-valued functions in $\mathrm{H}^{\frac{1}{2}}\left(\mathbb{R}^{3}, \mathbb{R}\right)$, corresponds to the identification

$$
\Gamma \cong \mathrm{H}^{\frac{1}{2}}\left(\mathbb{R}^{3}, \mathbb{R}^{2}\right)
$$

Note that $\psi^{(1)}$ and $\psi^{(2)}$ are canonically conjugate variables. In this paper, we use $\mathrm{H}^{\frac{1}{2}}\left(\mathbb{R}^{3}, \mathbb{R}^{2}\right)$ as the phase space, and, to distinguish the wave fields in this representation from $\psi \in \mathrm{H}^{\frac{1}{2}}\left(\mathbb{R}^{3}, \mathbb{C}\right)$, we use the boldface notation

$$
\left(\psi^{(1)}, \psi^{(2)}\right)=\boldsymbol{\psi} \in \mathrm{H}^{\frac{1}{2}}\left(\mathbb{R}^{3}, \mathbb{R}^{2}\right) .
$$

The tangent space, $\mathrm{T}_{\boldsymbol{\psi}} \Gamma$, to $\Gamma$ at a point $\boldsymbol{\psi} \in \Gamma$ is given by

$$
\left\{\boldsymbol{z}(x): \boldsymbol{z}(\cdot) \in \mathrm{H}^{\frac{1}{2}}\left(\mathbb{R}^{3}, \mathbb{R}^{2}\right), \boldsymbol{\psi}+\boldsymbol{z} \in \Gamma\right\} .
$$

Hence

$$
\mathrm{T}_{\boldsymbol{\psi}} \Gamma=\mathrm{H}^{\frac{1}{2}}\left(\mathbb{R}^{3}, \mathbb{R}^{2}\right) .
$$

A section of the tangent bundle $\mathrm{T} \Gamma$ is a vector field, i.e., an assignment of a vector $\boldsymbol{z}_{\boldsymbol{\psi}} \in \mathrm{T}_{\boldsymbol{\psi}} \Gamma$ to each point $\boldsymbol{\psi} \in \Gamma$ that depends continuously on $\boldsymbol{\psi}$.

The phase space carries a natural metric, $(\cdot, \cdot)_{\mathrm{L}^{2}\left(\mathbb{R}^{3}, \mathbb{R}^{2}\right), \boldsymbol{\psi}}$ : For $\boldsymbol{u}=\left(u_{\boldsymbol{\psi}}^{(1)}, u_{\boldsymbol{\psi}}^{(2)}\right), \boldsymbol{w}=$ $\left(w_{\boldsymbol{\psi}}^{(1)}, w_{\boldsymbol{\psi}}^{(2)}\right)$ in $\mathrm{T}_{\boldsymbol{\psi}} \Gamma, \boldsymbol{\psi} \in \Gamma$, we set

$$
(\boldsymbol{u}, \boldsymbol{w})_{2} \equiv(\boldsymbol{u}, \boldsymbol{w})_{\mathrm{L}^{2}\left(\mathbb{R}^{3}, \mathbb{R}^{2}\right), \boldsymbol{\psi}}:=\int_{\mathbb{R}^{3}}\left(u_{\boldsymbol{\psi}}^{(1)} w_{\boldsymbol{\psi}}^{(1)}+u_{\boldsymbol{\psi}}^{(2)} w_{\boldsymbol{\psi}}^{(2)}\right) \mathrm{d} x
$$

Furthermore, $\Gamma$ carries a complex structure denoted by $J$ : For $\boldsymbol{z} \in \mathrm{T}_{\boldsymbol{\psi}} \Gamma$, we set

$$
J \boldsymbol{z}=\left(z^{(2)},-z^{(1)}\right) .
$$


Of course, $\Gamma$ is symplectic with symplectic 2 -form given by

$$
\omega(\boldsymbol{u}, \boldsymbol{w}):=\int_{\mathbb{R}^{3}}\left(u^{(2)} w^{(1)}-u^{(1)} w^{(2)}\right) \mathrm{d} x,
$$

for $\boldsymbol{u}, \boldsymbol{w}$ in $\mathrm{T}_{\psi} \Gamma$. We observe that

$$
\omega(\boldsymbol{u}, \boldsymbol{w})=-(\boldsymbol{u}, J \boldsymbol{w})_{2} .
$$

In what follows we also consider a sub-space, $\tilde{\Gamma} \subset \Gamma$, given by

$$
\tilde{\Gamma}:=\left\{\boldsymbol{\psi} \in \Gamma:|x|^{1 / 2} \boldsymbol{\psi} \in \mathrm{L}^{2}\right\}
$$

and equipped with the norm

$$
\|\boldsymbol{u}\|_{\tilde{\Gamma}}^{2}:=\|\boldsymbol{u}\|_{\mathrm{H}^{\frac{1}{2}}}^{2}+\varepsilon\left\||x|^{1 / 2} \boldsymbol{u}\right\|_{2}^{2},
$$

where $\varepsilon$ is defined in (1.10). By the proof of Lemma 3 in [12], we find that if $\boldsymbol{\psi}_{0} \in \tilde{\Gamma}$, then $\boldsymbol{\psi}(\cdot, t) \in \tilde{\Gamma}$. See also Proposition 7.5.

The Hartree energy functional $\mathcal{H}_{V}$, the mass $\mathcal{N}$, and the momentum functional $\mathcal{P}$ (see (1.2)-(1.4)) have the form

$$
\begin{gathered}
\mathcal{H}_{V}(\boldsymbol{\psi}):=\frac{1}{2}\left(\boldsymbol{\psi},\left(\sqrt{-\Delta+m^{2}}-m\right) \boldsymbol{\psi}\right)_{2}+\frac{1}{2}(\boldsymbol{\psi}, V \boldsymbol{\psi})_{2}-\frac{1}{4}\left(\frac{1}{|x|} *|\boldsymbol{\psi}|^{2},|\boldsymbol{\psi}|^{2}\right)_{2}, \\
\mathcal{N}(\boldsymbol{\psi}):=\frac{1}{2}\|\boldsymbol{\psi}\|_{2}^{2}, \\
\mathcal{P}(\boldsymbol{\psi}):=\frac{1}{2}(\boldsymbol{\psi}, J \nabla \boldsymbol{\psi})_{2} .
\end{gathered}
$$

These functionals are well defined on $\Gamma=\mathrm{H}^{\frac{1}{2}}\left(\mathbb{R}^{3} ; \mathbb{R}^{2}\right)$.

We claim that (1.1) is the Hamiltonian equation of motion corresponding to the Hamiltonian $\mathcal{H}_{V}(\boldsymbol{\psi})$, given in (2.12). This is verified by noticing that the equation

$$
\partial_{t} \boldsymbol{\psi}=J_{\mathcal{H}_{V}^{\prime}}^{\prime}(\boldsymbol{\psi})
$$

is equivalent to (1.1).

If $V \equiv 0$, then the Hamiltonian is invariant under spatial translations $x \mapsto x+a$. The corresponding conserved quantity is the momentum $\mathcal{P}(\boldsymbol{\psi})$ defined above. For bounded $V, \mathcal{P}$ satisfies an Ehrenfest identity

$$
d_{t} \mathcal{P}(\boldsymbol{\psi})=-\frac{1}{2}(\boldsymbol{\psi} \nabla V, \boldsymbol{\psi})_{2} .
$$

This identity was shown in [8, App. A] for the nonlinear Schrödinger equation, but the proof carries over to (1.1).

We define a functional $\mathcal{E}_{v, \mu}$ as

$$
\mathcal{E}_{v, \mu}(\boldsymbol{\psi}):=\mathcal{H}_{V=0}(\boldsymbol{\psi})+\mu \mathcal{N}(\boldsymbol{\psi})-v \cdot \mathcal{P}(\boldsymbol{\psi}),
$$


which is given, more explicitly, by

$\mathcal{E}_{v, \mu}(\boldsymbol{\psi})=\frac{1}{2}\left(\boldsymbol{\psi},\left(\sqrt{-\Delta+m^{2}}-m\right) \boldsymbol{\psi}\right)_{2}+\frac{\mu}{2}\|\boldsymbol{\psi}\|_{2}^{2}-\frac{1}{2} v \cdot(\boldsymbol{\psi}, J \nabla \boldsymbol{\psi})_{2}-\frac{1}{4}\left(|\boldsymbol{\psi}|^{2}, \frac{1}{|x|} *|\boldsymbol{\psi}|^{2}\right)_{2}$

This functional plays a key role in this paper, and we briefly discuss its properties. The ground states $\varphi_{v, \mu}=\left(\operatorname{Re} \varphi_{v, \mu}, \operatorname{Im} \varphi_{v, \mu}\right)$, i.e., solutions to eq. (1.7) are solutions to

$$
\mathcal{E}_{v, \mu}^{\prime}\left(\boldsymbol{\varphi}_{v, \mu}\right)=0
$$

Its Hessian, $L_{v, \mu}:=\mathcal{E}_{v, \mu}^{\prime \prime}\left(\boldsymbol{\varphi}_{v, \mu}\right)$, is given by the linear symmetric operator:

$$
L_{v, \mu}:=\left(\begin{array}{ll}
L_{11} & L_{12} \\
L_{21} & L_{22}
\end{array}\right)
$$

where, for $\xi \in \mathrm{H}^{\frac{1}{2}}\left(\mathbb{R}^{3}, \mathbb{R}\right)$,

$$
\begin{aligned}
L_{11, v, \mu} \xi & :=\left(\sqrt{-\Delta+m^{2}}-m+\mu-\frac{1}{|x|} *\left|\boldsymbol{\varphi}_{v, \mu}\right|^{2}\right) \xi-\left(\frac{2}{|x|} *\left(\xi \varphi_{v, \mu}^{(1)}\right)\right) \varphi_{v, \mu}^{(1)}, \\
L_{12, v, \mu} \xi & :=-v \cdot \nabla \xi-\left(\frac{2}{|x|} *\left(\xi \varphi_{v, \mu}^{(2)}\right)\right) \varphi_{v, \mu}^{(1)}, \\
L_{21, v, \mu} \xi & :=v \cdot \nabla \xi-\left(\frac{2}{|x|} *\left(\xi \varphi_{v, \mu}^{(1)}\right)\right) \varphi_{v, \mu}^{(2)}, \\
L_{22, v, \mu} \xi & :=\left(\sqrt{-\Delta+m^{2}}-m+\mu-\frac{1}{|x|} *\left|\varphi_{v, \mu}\right|^{2}\right) \xi-\left(\frac{2}{|x|} *\left(\xi \varphi_{v, \mu}^{(2)}\right)\right) \varphi_{v, \mu}^{(2)} .
\end{aligned}
$$

We find $\nabla \boldsymbol{\varphi}_{v, \mu}$ and $J \boldsymbol{\varphi}_{v, \mu}$ to be elements of the kernel of $L_{v, \mu}, \operatorname{Ker}\left(L_{v, \mu}\right)$, because $\mathcal{E}_{v, \mu}$ is invariant under gauge transformations and translations. For $v=0, L_{v, \mu}$ reduces to $L_{\mu}:=\operatorname{diag}\left(L_{11, \mu}, L_{22, \mu}\right)$. A key assumption underlying our analysis is

Assumption 2.1. Let $L_{\mu}=\operatorname{diag}\left(L_{11, \mu}, L_{22, \mu}\right)$ be defined as above. We assume that the dimension of the null space of $L_{11, \mu}$ is three, i.e.,

$$
\operatorname{dim} \operatorname{Ker}\left(L_{11, \mu}\right)=3, \text { for } \mu>0 \text {. }
$$

In [10] this assumption is verified numerically for some $\mu>0$, following [5].

\section{The main theorem}

In this section we state our assumptions and the main theorem. We then present an outline of the proof, which is implemented in the remaining sections of this paper.

Given a number $\varepsilon>0$, we require that the external potential $V \in \mathrm{C}^{3}$ satisfies

$$
\sup _{x}\left|\partial_{x}^{\alpha} V\right| \leq C \varepsilon^{|\alpha|}, \text { for }|\alpha| \leq 3
$$

where $\alpha$ is a multi-index and $C$ is a constant. 
The ground state $\varphi_{v, \mu}$ is not known to be unique modulo phase transformations and translations. We therefore single out a particular class of solutions to (2.19), also denoted by $\boldsymbol{\varphi}_{v, \mu}$, near a spherically symmetric minimizer $\boldsymbol{\varphi}_{\mu}:=\boldsymbol{\varphi}_{v=0, \mu}$ by the use of an implicit function theorem and Assumption 2.1. As we will see, there is a maximal number $r_{0}$ and a maximal open interval $I_{0}$, with $0<r_{0}<1$, such that for $|v|<r_{0}$ and $\mu \in I_{0}, \boldsymbol{\varphi}_{v, \mu}$ solves (2.19). The construction, as well as several properties of these functions are given in Proposition 4.3. For any $r<r_{0}$ and an open non-empty interval $I \subset I_{0}$, let $\mathrm{Z}(r, I)$ be defined by

$$
\mathrm{Z}(r, I):=\mathbb{R}^{3} \times \mathrm{B}_{r}(0) \times[0,2 \pi) \times I,
$$

where $\mathrm{B}_{r}(0)=\left\{v \in \mathbb{R}^{3}:|v|<r\right\}$. Consider the manifold

$$
\mathrm{M}(\mathrm{Z}):=\left\{\mathrm{e}^{-\vartheta J} \boldsymbol{\varphi}_{v, \mu}(\cdot-y):(y, v, \vartheta, \mu) \in \mathrm{Z}\right\},
$$

The soliton manifold is defined by $\mathrm{M}_{0}:=\mathrm{M}\left(\mathrm{Z}_{0}\right)$ with $\mathrm{Z}_{0}:=\mathrm{Z}\left(r_{0}, I_{0}\right)$, where $r_{0}$ is the maximal speed and $I_{0}$ the maximal frequency interval such that $\boldsymbol{\varphi}_{v, \mu}$ is well defined in the sense of Proposition 4.3. Thus, $\mathrm{Z}_{0}$ is the parameter space for $\mathrm{M}_{0}$. Furthermore, we introduce a symbol, $\zeta$, for a point in $\mathrm{Z}_{0}$ by

$$
\zeta:=(y, v, \vartheta, \mu)
$$

and note that each point in $\mathrm{M}_{0}$ can be described by $\boldsymbol{\varphi}_{\zeta}$, where

$$
\boldsymbol{\varphi}_{\zeta}(x):=\mathrm{e}^{-\vartheta J} \boldsymbol{\varphi}_{v, \mu}(x-y) .
$$

The tangent space to $M_{0}$ at $\boldsymbol{\varphi}_{\zeta}$ is given by

$$
\mathrm{T}_{\boldsymbol{\varphi}_{\zeta}} \mathrm{M}_{0}:=\operatorname{span}\left(\nabla_{y} \boldsymbol{\varphi}_{\zeta}, \nabla_{v} \boldsymbol{\varphi}_{\zeta}, J \boldsymbol{\varphi}_{\zeta}, \partial_{\mu} \boldsymbol{\varphi}_{\zeta}\right)
$$

We can now state our main theorem.

Theorem 3.1. Suppose that Assumption 2.1 is satisfied. Let $r_{0}, I_{0}$, and $\boldsymbol{\varphi}_{v, \mu}$ be as above. Let the external potential $V$ satisfy (3.1). Then there is an open non-empty interval $\tilde{I}$, with ${ }^{2} \tilde{I} \Subset I_{0}$ as well as numbers $0<\tilde{r}<r_{0}$ and $\varepsilon>0$, with $\varepsilon$ sufficiently small, such that the following holds. If the initial condition $\boldsymbol{\psi}_{0} \in \tilde{\Gamma}$ for (2.15) satisfies

$$
\left\|\boldsymbol{\psi}_{0}-\boldsymbol{\varphi}_{\zeta^{(0)}}\right\|_{\tilde{\Gamma}} \leq \varepsilon
$$

for some $\zeta^{(0)} \in \tilde{\mathrm{Z}}$, with $\tilde{\mathrm{Z}}:=\mathrm{Z}(\tilde{r}, \tilde{I})$, and $\|\cdot\|_{\tilde{\Gamma}}$ as defined in $(2.11)$, then the solution $\boldsymbol{\psi}$ of (2.15) is of the form

$$
\boldsymbol{\psi}(x, t)=\mathrm{e}^{-\vartheta J}\left(\boldsymbol{\varphi}_{v, \mu}(x-y)+\boldsymbol{\xi}(x-y, t)\right),
$$

where $y, v, \vartheta, \mu$ are time-dependent functions and

$$
\|\boldsymbol{\xi}\|_{\tilde{\Gamma}} \leq C \varepsilon .
$$

\footnotetext{
${ }^{2}$ Here $A \Subset B$ is defined by $\bar{A} \subset B, \bar{A}$ compact.
} 
Moreover, we have that

$$
\begin{aligned}
\left|d_{t} \mathcal{N}\left(\boldsymbol{\varphi}_{v, \mu}\right)\right| & \leq C \varepsilon^{2}, & & \left|d_{t} \mathcal{P}\left(\boldsymbol{\varphi}_{v, \mu}\right)+\mathcal{N}\left(\boldsymbol{\varphi}_{v, \mu}\right) \nabla V(y)\right| \leq C \varepsilon^{2}, \\
|\dot{y}-v| & \leq C \varepsilon^{2}, & & |\dot{\vartheta}+\mu-V(y)| \leq C \varepsilon^{2},
\end{aligned}
$$

for times $0 \leq t \leq C / \varepsilon$, where $C>0$ is some constant.

The requirement that the initial condition be 'close' in norm to $\mathrm{M}(\tilde{\mathrm{Z}})$ can, as in e.g., $[8,16,4,3]$, be used to introduce an additional small parameter, $\varepsilon_{0}$, to separate the two scales mentioned in the introduction. But to simplify the exposition in the present paper, we assume this distance also to be bounded by $\varepsilon$.

Next, we outline the essential parts of the proof of Theorem 3.1. Above, we have introduced $r_{0}, I_{0}$ and $\tilde{r}, \tilde{I}$. In the process of the proof, we will find a nested sequence of manifolds: There are numbers $r_{j}$ and open non-empty intervals $I_{j}$, for $j=0, \ldots, 3$, with $r_{3}:=\tilde{r}$ and $I_{3}:=\tilde{I}$. Here $0<r_{j+1}<r_{j}$ and $\bar{I}_{j+1} \subset I_{j}$ for $j=0,1,2$. The corresponding parameter domains are $\mathrm{Z}_{j}:=\mathrm{Z}\left(r_{j}, I_{j}\right)$ corresponding to soliton submanifolds $\mathrm{M}_{j}:=$ $\mathrm{M}\left(\mathrm{Z}_{j}\right)$. One of the constraints on $\varepsilon$ is that it has to be smaller than the distance between the boundaries of the nested sequence of the manifolds. These distances are indicated in Figure 3.1; see also Remark 6.2.

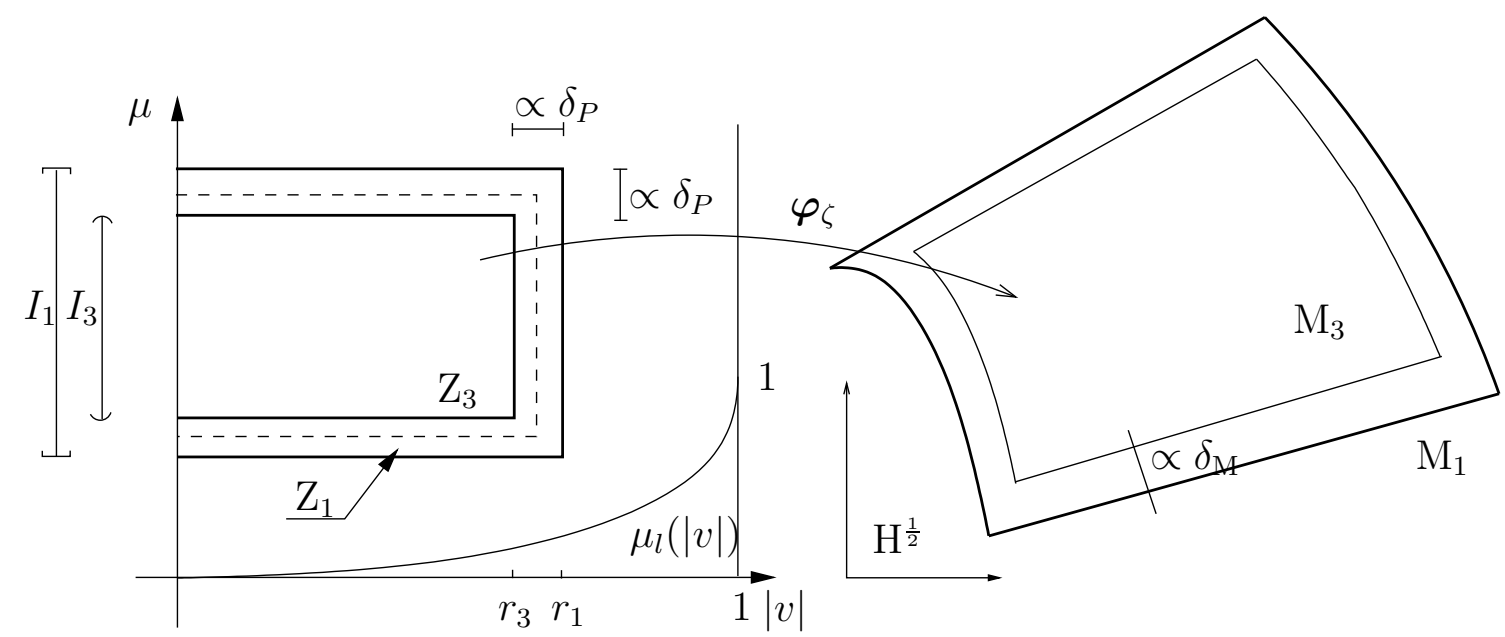

Figure 3.1: The left figure displays a schematic view of the parameter spaces $Z_{1}$ and $\mathrm{Z}_{3}$. The function $\mu_{l}(|v|)$ is defined in Proposition 4.3 and represents the lower bound on $\mu$. The dotted line indicates $\mathrm{Z}_{2} ; \delta_{M}$ and $\delta_{P}$ are the distance between the manifolds and upper bounds on $\varepsilon$, see Remark 6.2.

Once we derive the properties of the ground state (Sect. 4), we need to derive the finite-dimensional dynamics expressed by (3.10)-(3.11). To do this, we show that the symplectic form is non-degenerate on $\mathrm{M}_{0}$ by using the symmetry properties of $\boldsymbol{\varphi}_{v, \mu}$, and its derivatives; see Section 5. This non-degeneracy of the symplectic form on $\mathrm{M}_{0}$ is the key fact to show the existence of a skew (or symplectically) orthogonal decomposition of $\boldsymbol{\psi}$ in a tubular neighborhood $U_{\delta}\left(\mathrm{Z}_{2}\right)$ around $\mathrm{M}_{2}$. That is, there is a unique map 
$\varsigma: U_{\delta}\left(\mathrm{Z}_{2}\right) \rightarrow \mathrm{Z}_{1}$ such that

$$
\boldsymbol{\psi}(x)=\boldsymbol{\varphi}_{\varsigma(\boldsymbol{\psi})}(x)+\mathrm{e}^{-\vartheta(\boldsymbol{\psi}) J} \boldsymbol{\xi}(x-y(\boldsymbol{\psi}), t)
$$

and

$$
\omega\left(\boldsymbol{\psi}-\boldsymbol{\varphi}_{\varsigma(\psi)}, \boldsymbol{z}\right)=0, \quad \forall \boldsymbol{z} \in \mathrm{T}_{\boldsymbol{\varphi}_{\varsigma(\psi)}} \mathrm{M}_{1} .
$$

This result is proven in Section 6 .

The existence of the decomposition (3.12)-(3.13) enables us to 'change variables' from $\boldsymbol{\psi}$ to $(\zeta, \boldsymbol{\xi})$, where $\zeta(t):=\varsigma(\boldsymbol{\psi}(\cdot, t))$. The proof of the uniqueness of the decomposition also gives estimates for the distance between the initial parameter point $\zeta^{(0)}$, (see $(3.7)$ ) and the starting point is given by the decomposition $\varsigma\left(\boldsymbol{\psi}_{0}\right)$ as well as the distance between $\boldsymbol{\varphi}_{\zeta^{(0)}}$ and $\boldsymbol{\varphi}_{\varsigma\left(\psi_{0}\right)}$. Figure 3.2 shows what we have in mind. (See also Figure 6.1).

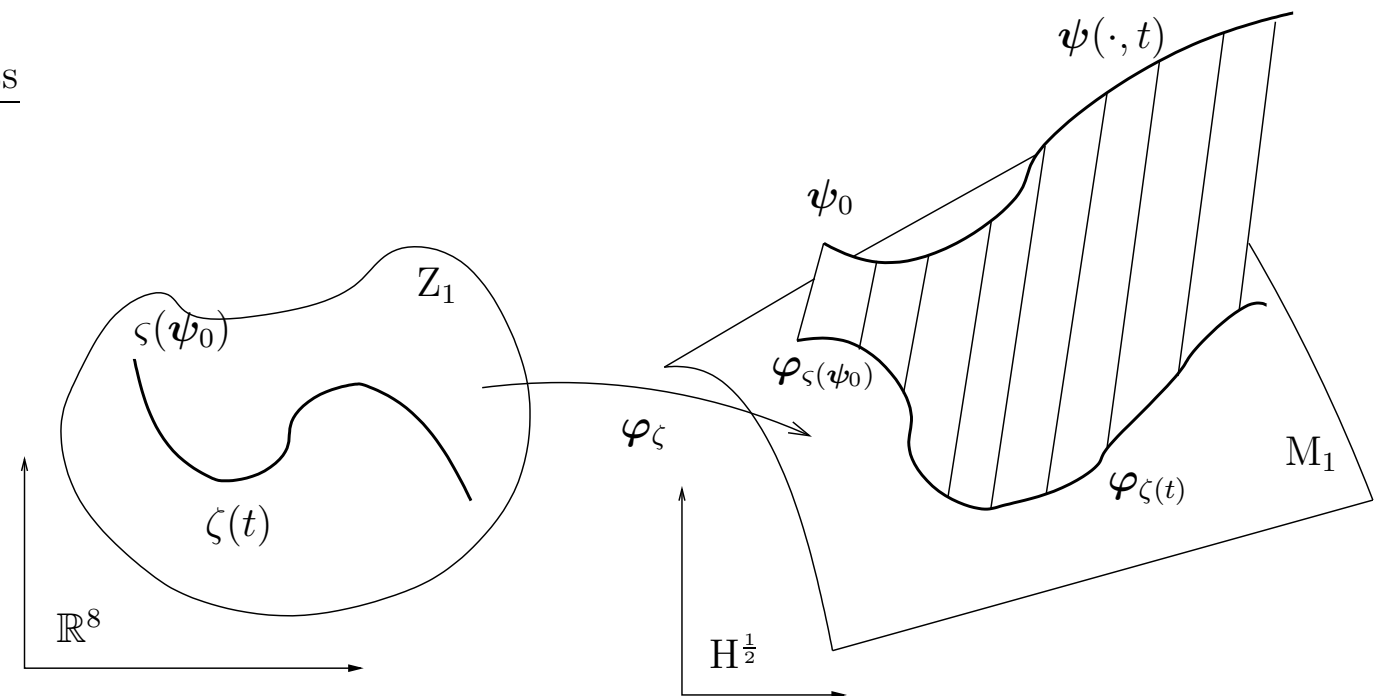

Figure 3.2: The dynamics of $\boldsymbol{\psi}, \boldsymbol{\varphi}_{\zeta}$ and $\zeta(t):=\varsigma(\boldsymbol{\psi}(\cdot, t))$. The diagonal lines connecting the trajectories of $\boldsymbol{\varphi}_{\zeta}$ and $\boldsymbol{\psi}$ indicate the skew-orthogonal projection.

In Section 7, we insert the symplectically orthogonal decomposition into the equation of motion (2.15). We then use the symplectic form to project out the finite-dimensional equations for $\zeta$, which schematically take the form

$$
\dot{\zeta}=X(\zeta)+Y(\zeta, \boldsymbol{\xi})
$$

We then show that

$$
|Y(\zeta, \boldsymbol{\xi})| \leq C\left(\varepsilon^{2}+\|\boldsymbol{\xi}\|_{\mathrm{H}^{\frac{1}{2}}}|\alpha|+\|\boldsymbol{\xi}\|_{\mathrm{H}^{\frac{1}{2}}}^{2}+\|\boldsymbol{\xi}\|_{\mathrm{H}^{\frac{1}{2}}}^{3}\right)
$$

and $\alpha=(\dot{y}-v, \dot{v}, \mu-\dot{\vartheta}-V(y), \dot{\mu})$ is bounded by

$$
|\alpha| \leq C(\varepsilon+|Y|) .
$$


Finally, $\dot{v}$ and $\dot{\mu}$ are shown to be of size $C \varepsilon$, whereas the full dynamics $|\dot{\zeta}-X(\zeta)|$ is bounded by $C \varepsilon^{2}$. Thus, by (3.14), we have found the finite-dimensional dynamics, i.e., the equation of motion for $\zeta$.

The equation for the perturbation $\boldsymbol{\xi}$ takes the form

$$
\partial_{t} \boldsymbol{\xi}=L_{v, \mu} \boldsymbol{\xi}+\mathcal{M}_{\boldsymbol{\varphi}_{v, \mu}}(\boldsymbol{\xi})+\ldots
$$

The procedure in Sections 5-7 of the paper is collected in Figure 3.3.

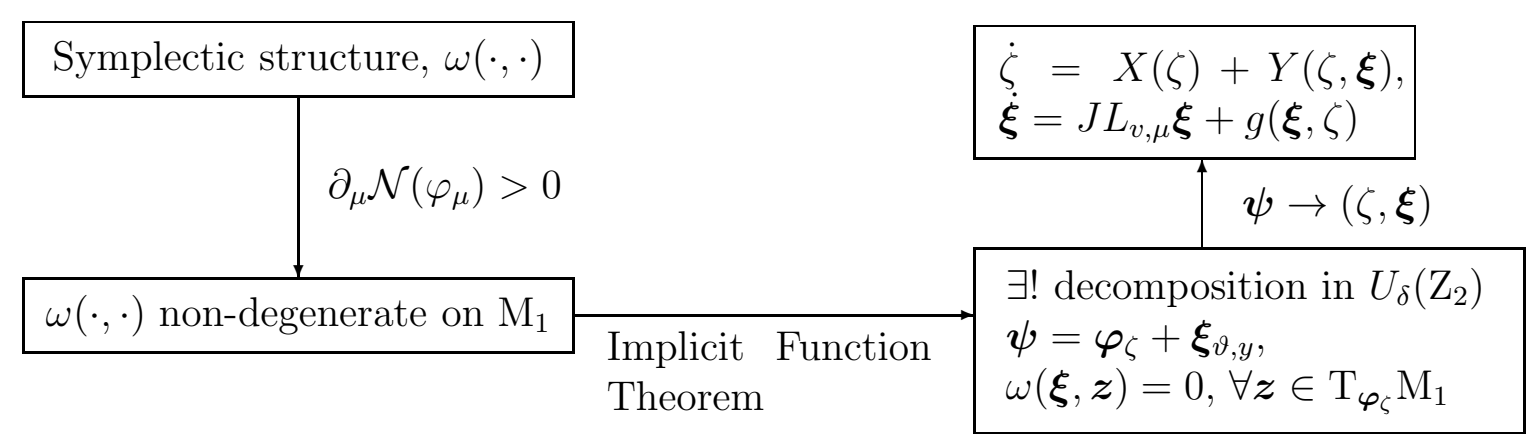

Figure 3.3: The way to the dynamics of $(\zeta, \boldsymbol{\xi})$. A schematic representation of Sections 57. $\boldsymbol{\xi}_{\vartheta, y}(x, t):=\mathrm{e}^{-\vartheta J} \boldsymbol{\xi}(x+y, t), g(\boldsymbol{\xi}, \zeta)$ is the coupling term. For an explicit form see (7.6).

The next step in the proof of our main theorem is to control the $\boldsymbol{\xi}$-term. This is done in three steps as follows. We first show, in Section 8, that the dynamics of $\mathcal{Q}:=(\boldsymbol{\xi},|x| \boldsymbol{\xi})_{2}$ is well behaved and satisfies the inequality

$$
\mathcal{Q}(t) \leq \mathcal{Q}(0)+C\|\boldsymbol{\xi}\|_{\mathrm{H}^{\frac{1}{2}}}
$$

for times $t \leq T_{1}$ and such that the symplectic decomposition is valid.

To control the $\mathrm{H}^{\frac{1}{2}}$-norm of $\boldsymbol{\xi}$, we introduce a Lyapunov functional, $\mathcal{S}(t)$, which is a linear combination of $\mathcal{H}_{V}, \mathcal{N}$ and $\mathcal{P}$ at $\boldsymbol{\psi}$, minus the same quantities at $\boldsymbol{\varphi}_{\zeta}$, that is

$$
\mathcal{S}(t):=(\mu-V(y))\left(\mathcal{N}(\boldsymbol{\psi})-\mathcal{N}\left(\boldsymbol{\varphi}_{\zeta}\right)\right)+v \cdot\left(\mathcal{P}(\boldsymbol{\psi})-\mathcal{P}\left(\boldsymbol{\varphi}_{\zeta}\right)\right)+\mathcal{H}_{V}(\boldsymbol{\psi})-\mathcal{H}_{V}\left(\boldsymbol{\varphi}_{\zeta}\right)
$$

In Section 9, we bound this functional from below in terms of $\|\boldsymbol{\xi}\|_{\mathrm{H}^{\frac{1}{2}}}$, plus small perturbation terms. To do this, we use the spectral properties of $L_{v, \mu}$.

In Section 10, we bound this functional from above in terms of small quantities, (i.e., powers of $\left.\|\boldsymbol{\xi}\|_{\mathrm{H}^{\frac{1}{2}}},|Y|\right)$, for times $t \leq T_{2}$ and such that the decomposition is valid. To do this, we use the that $\mathcal{S}(t)$ is "almost conserved".

In the last Section 11, we combine our estimates on $\|\boldsymbol{\xi}\|_{\mathrm{H}^{\frac{1}{2}}}, \mathcal{Q}$ and $|Y|$. The arguments in Section 11 prove the main theorem. 


\section{Properties of ground states}

In this section, we derive properties of the elements of $\mathrm{M}_{0}$.

Let $R_{v, \theta}$ be a rotation of angle $\theta$ in $x$ around the $v$-direction. Let $S_{v}$ be reflection in $x$ along the $v$-direction.

Definition 4.1. Let $v \in \mathbb{R}^{3}$ with $v \neq 0$. A function $\boldsymbol{w}=\left(w^{(1)}, w^{(2)}\right): \mathbb{R}^{3} \rightarrow \mathbb{R}^{2}$ is called $v$-symmetric if

$$
R_{v, \theta} \boldsymbol{w}=\boldsymbol{w}, \text { for all } \theta \in[0,2 \pi) \text {, and } S_{v} \boldsymbol{w}=\left(w^{(1)},-w^{(2)}\right) .
$$

Analogously for any direction, say $\hat{e}_{3}$, we call a function $\hat{e}_{3}$-symmetric if it satisfies Definition 4.1 with $v$ replaced with $\hat{e}_{3}$.

Definition 4.2. Let $f: \mathbb{R} \rightarrow \mathbb{R}$. If $f$ is an even function then we write $f \sim(e)$ and if it is odd $f \sim(o)$.

For functions $\boldsymbol{w}: \mathbb{R}^{3} \rightarrow \mathbb{R}^{2}$ we use $\sim$ likewise. For example, $\boldsymbol{w} \sim($ eee, eeo $)$ means that the first component of $\boldsymbol{w}$ is even in its three coordinate directions and the second component is even in its first two coordinate directions and odd in the last. We write $\boldsymbol{w} \sim \boldsymbol{u}$ to indicate that $\boldsymbol{w}$ and $\boldsymbol{u}$ have the same reflection symmetries.

We have the result:

Proposition 4.3. Suppose Assumption 2.1 is satisfied and let $m>0$. Define $\mu_{l}(|v|):=$ $\left(1-\sqrt{1-|v|^{2}}\right) m$. Then there is a number $r_{0} \in(0,1)$ and an open non-empty interval $I_{0}:=\left(\mu_{l 0}, \mu_{h 0}\right)$, with $\mu_{l 0}>\mu_{l}(|v|)$ such that for all $\mu \in I_{0}$ and $|v| \leq r_{0}<1$ we have:

(i). there is a function $(v, \mu) \mapsto \boldsymbol{\varphi}_{v, \mu} \in \mathrm{C}^{\infty}\left(B_{r_{0}} \times I_{0}, \mathrm{H}^{1}\right)$ that solves (2.19) and $\boldsymbol{\varphi}_{v, \mu} \in$ $\mathrm{H}^{s}$ for all $s \geq 1$. Moreover, we have $\boldsymbol{\varphi}_{\mu}:=\boldsymbol{\varphi}_{0, \mu}$, with $\boldsymbol{\varphi}_{\mu}=\left(\varphi_{\mu}, 0\right)$ and $\varphi_{\mu}$ is spherically symmetric and positive;

(ii). $\boldsymbol{\varphi}_{v, \mu}$ and $\partial_{\mu} \boldsymbol{\varphi}_{v, \mu}$ are $v$-symmetric, and $\partial_{v_{j}} \boldsymbol{\varphi}_{v, \mu} \sim J \partial_{x_{j}} \boldsymbol{\varphi}_{v, \mu}$;

(iii). $\boldsymbol{\varphi}_{v, \mu}, \partial_{x_{j}} \boldsymbol{\varphi}_{v, \mu}, \partial_{\mu} \boldsymbol{\varphi}_{v, \mu}$, and $\partial_{v_{k}} \boldsymbol{\varphi}_{v, \mu}$ are pointwise exponentially localized;

(iv). 'Stability condition', $\partial_{\mu}\left\|\boldsymbol{\varphi}_{v, \mu}\right\|_{2}^{2}>c$, where $c$ is independent of $v \in\left\{v:|v| \leq r_{0}\right\}$, $\mu \in I_{0}$. Furthermore $\boldsymbol{\varphi}_{\mu}$ is a minimizer of $\mathcal{E}_{0,0}$ subject to $\mathcal{N}$ constant;

(v). $L_{v, \mu}:=\mathcal{E}_{v, \mu}^{\prime \prime}\left(\boldsymbol{\varphi}_{v, \mu}\right)$ has one negative eigenvalue. Moreover, $\operatorname{dim} \operatorname{Ker}\left(L_{v, \mu}\right)=4$; there is a spectral gap between zero and its next spectral point; and the essential spectrum starts at $\mu-\mu_{l}(|v|)>0$.

The ground state $\boldsymbol{\varphi}_{\mu}$ constructed in Proposition 4.3((i)) is used to define the soliton manifold $\mathrm{M}_{0}:=\mathrm{M}\left(\mathrm{Z}_{0}\right)$, where $\mathrm{Z}_{0}=\mathrm{Z}\left(r_{0}, I_{0}\right)$ in (3.3). It is convenient to use $\zeta:=$ $(y, v, \vartheta, \mu)$ as a point in $\mathrm{Z}_{0}$, and subsequently (as in (3.5))

$$
\boldsymbol{\varphi}_{\zeta}(x):=\mathrm{e}^{-\vartheta J} \boldsymbol{\varphi}_{v, \mu}(x-y)
$$


These ground states $\boldsymbol{\varphi}_{\zeta}$ satisfy the Euler-Lagrange equation

$$
\mathcal{E}_{v, \mu}^{\prime}\left(\boldsymbol{\varphi}_{\zeta}\right)=0
$$

and taking derivatives of (4.3) with respect to $y$ and $\vartheta$ evaluated at $(y, \vartheta)=(0,0)$ yield

$$
L_{v, \mu} \partial_{x_{j}} \boldsymbol{\varphi}_{v, \mu}=0, \quad L_{v, \mu} J \boldsymbol{\varphi}_{v, \mu}=0 .
$$

Thus $\partial_{x_{j}} \boldsymbol{\varphi}_{v, \mu}$ and $J \boldsymbol{\varphi}_{v, \mu}$ are zero modes to $L_{v, \mu}$. The derivatives of Eq. (4.3) with respect to $v_{j}$ and $\mu$, respectively, at $y=0, \vartheta=0$ lead to

$$
L_{v, \mu} \partial_{v_{j}} \boldsymbol{\varphi}_{v, \mu}=J \partial_{x_{j}} \boldsymbol{\varphi}_{v, \mu}, \quad L_{v, \mu} \partial_{\mu} \boldsymbol{\varphi}_{v, \mu}=-\boldsymbol{\varphi}_{v, \mu} .
$$

Therefore $\partial_{v_{j}} \boldsymbol{\varphi}_{v, \mu}$ and $\partial_{\mu} \boldsymbol{\varphi}_{v, \mu}$ are zero modes to $\left(J L_{v, \mu}\right)^{2}$. The tangent vectors of $\mathrm{M}_{0}$ are hence in the generalized null space of $J L_{v, \mu}$ (compare with e.g., [35]). Below, we prove part (i) $-(\mathrm{v})$ of Proposition 4.3 and the completion of the proof can be found in Subsection 4.4 .

\subsection{Proof of Proposition 4.3(i),(ii)}

The functions $\boldsymbol{\varphi}_{v, \mu}$ will be constructed as a class of solutions to the Euler-Lagrange equation (2.19), starting from an unboosted, with $v=0$, minimizer $\varphi_{0}:=\left(\varphi_{0}, 0\right)$ at frequency $\mu_{0}$. Here, $\varphi_{0}$ is a spherically symmetric positive minimizer to $\mathcal{E}_{0,0}$ at constant $\mathcal{N}$ from [23] and [9].

A non-zero velocity $v$ breaks the rotation symmetry of the map $\mathcal{E}_{v, \mu}^{\prime}$. Without loss of generality we pick a preferred direction, $\hat{e}_{3}$, the unit vector parallel to the $x_{3}$-axis, and choose coordinates so that $v=\tilde{v} \hat{e}_{3}$. Let $R_{3, \theta}$ be the spatial rotation around $\hat{e}_{3}$ of angle $\theta, S_{3}$ the spatial reflection along $\hat{e}_{3}$ and let $K$ be the matrix

$$
K=\left(\begin{array}{cc}
1 & 0 \\
0 & -1
\end{array}\right)
$$

The $\hat{e}_{3}$-symmetric Sobolev space, $\mathrm{H}_{\hat{e}_{3}}^{s}$, of order $s$ is defined as

$$
\mathrm{H}_{\hat{e}_{3}}^{s}:=\left\{\boldsymbol{\psi} \in \mathrm{H}^{s}\left(\mathbb{R}^{3}, \mathbb{R}^{2}\right): R_{3, \theta} \boldsymbol{\psi}=\boldsymbol{\psi}, \forall \theta \in[0,2 \pi), K S_{3} \boldsymbol{\psi}=\boldsymbol{\psi}\right\},
$$

where $R_{3, \theta} \boldsymbol{\psi}(x)=\boldsymbol{\psi}\left(R_{3, \theta} x\right)$.

Remark 4.4. That $\mathrm{H}_{\hat{e}_{3}}^{s}$ is a closed subspace of $\mathrm{H}^{s}\left(\mathbb{R}^{3}, \mathbb{R}^{2}\right)$ follows by noting that $R_{3, \theta}$ and $K S_{3}$ are bounded operators on $\mathrm{H}^{s}\left(\mathbb{R}^{3}, \mathbb{R}^{2}\right)$ and that $\mathrm{H}_{\hat{e}_{3}}^{s}=\cap_{\theta \in[0,2 \pi)} \operatorname{Ker}\left(() 1-R_{3, \theta}\right) \cap$ $\operatorname{Ker}\left(() 1-K S_{3}\right)$.

We recast Proposition 4.3((i)) and the first part of ((ii)) into:

Proposition 4.5. Suppose that Assumption 2.1 is satisfied. Let $\varphi_{0}, \mu_{0}$ and $\mathrm{H}_{\hat{e}_{3}}^{s}$, be as above, let $v:=\tilde{v} \hat{e}_{3}$, with $\tilde{v} \in \mathbb{R}$. Then there is an open neighborhood, $W \subset \mathbb{R} \times \mathbb{R}_{+}$, with $\left(0, \mu_{0}\right) \in W$, and a unique function $(\tilde{v}, \mu) \mapsto \boldsymbol{\varphi}_{\tilde{v} \hat{e}_{3}, \mu} \in \mathrm{C}^{\infty}\left(W, \mathrm{H}_{\hat{e}_{3}}^{1}\right)$ such that $\boldsymbol{\varphi}_{0 \hat{e}_{3}, \mu_{0}}=\boldsymbol{\varphi}_{0}$ and $\boldsymbol{\varphi}_{\tilde{v} \hat{e}, \mu}, \mu$ solves $\mathcal{E}_{\tilde{v} \hat{e}, \mu}^{\prime}(\boldsymbol{\psi})=0$ for all $(\tilde{v}, \mu) \in W$. In addition, $\boldsymbol{\varphi}_{\hat{v} \hat{e}_{3}, \mu}$ belongs to $\mathrm{H}^{s}$ for all $s \geq 1$. 
Remark 4.6. (a) A solution to $\mathcal{E}_{v, \mu}^{\prime}(\boldsymbol{\psi})=0$ when $v \neq 0$ points in arbitrary direction is obtained by rotating $\boldsymbol{\varphi}_{\tilde{v}_{\hat{e}}, \mu}$ in $x$ from $\hat{e}_{3}$ to $\hat{v}:=v /|v|$. See the proof of Corollary 4.7 for details. (b) The Sobolev space of order one of radially symmetric functions, $\mathrm{H}_{\mathrm{rad}}^{1}\left(\mathbb{R}^{3}, \mathbb{R}\right)$, is a scalar subspace of $\mathrm{H}_{\hat{e}_{3}}^{1}$. This ensures existence and uniqueness of a solution $\boldsymbol{\varphi}_{v=0, \mu}=$ $\boldsymbol{\varphi}_{\mu}=\left(\varphi_{\mu}, 0\right)$ to $\mathcal{E}_{0, \mu}^{\prime}(\boldsymbol{\psi})=0$, where $\varphi_{\mu} \in \mathrm{H}_{\mathrm{rad}}^{1}$. (c) $\mathcal{E}_{v, \mu}$ is invariant under translation and change of gauge. Thus, $\mathrm{e}^{\vartheta J} \boldsymbol{\varphi}_{v, \mu}(\cdot+y)$ for any $y \in \mathbb{R}^{3}, \vartheta \in[0,2 \pi)$ is also a solution to $(2.19)$.

Proposition 4.5 proves that $\boldsymbol{\varphi}_{\tilde{v} \hat{e}_{3}, \mu}, \partial_{\mu} \boldsymbol{\varphi}_{\tilde{v}_{\hat{e}}, \mu} \in \mathrm{H}_{\hat{e}_{3}}^{s}$. For arbitrary coordinates this implies that $\boldsymbol{\varphi}_{v, \mu}$ and $\partial_{\mu} \boldsymbol{\varphi}_{v, \mu}$ are $v$-symmetric. The reflection symmetries of $\partial_{v_{j}} \boldsymbol{\varphi}_{v, \mu}$ now follows from:

Corollary 4.7. Suppose that the Assumption 2.1 is satisfied. Then

$$
\partial_{v_{j}} \boldsymbol{\varphi}_{v, \mu} \sim J \partial_{x_{j}} \boldsymbol{\varphi}_{v, \mu}, j=1,2,3 .
$$

The corollary is proved at the end of this subsection.

Lemma 4.8. We have that

$$
(\tilde{v}, \mu, \boldsymbol{\psi}) \mapsto \mathcal{E}_{\tilde{v} \hat{e}_{3}, \mu}^{\prime}(\boldsymbol{\psi}) \in \mathrm{C}^{\infty}\left(\mathbb{R} \times \mathbb{R} \times \mathrm{H}_{\hat{e}_{3}}^{1}, \mathrm{H}_{\hat{e}_{3}}^{0}\right) .
$$

Proof of Proposition 4.5. Let $F(\tilde{v}, \mu, \boldsymbol{\psi}):=\mathcal{E}_{\tilde{v} \hat{e}_{3}, \mu}^{\prime}(\boldsymbol{\psi})$. To find solutions to the equation $F(\tilde{v}, \mu, \boldsymbol{\psi})=0$, we use the implicit function theorem in [6], which has three assumptions, that we now verify: That $F$ is $\mathrm{C}^{\infty}$ is shown by Lemma 4.8 . The equation $F=0$ has a solution $\left(0, \mu_{0}, \boldsymbol{\varphi}_{0}\right)$ and $\boldsymbol{\varphi}_{0} \in \mathrm{H}_{\hat{e}_{3}}^{1}$. The last condition is that $F_{\boldsymbol{\psi}}^{\prime}\left(0, \mu_{0}, \boldsymbol{\varphi}_{0}\right)=: L_{\mu_{0}}$, where $L_{\mu_{0}}=\operatorname{diag}\left(L_{11, \mu_{0}}, L_{22, \mu_{0}}\right)$ is invertible. We have that $L_{22, \mu_{0}} \geq 0$ and $L_{22, \mu_{0}} \varphi_{0}=0$, the zero eigenvalue is non-degenerate since $\mathrm{e}^{-t L_{22, \mu_{0}}}$ is positivity improving. (This follows from the explicit kernel for $\mathrm{e}^{-t \sqrt{-\Delta+m^{2}}}$ and Trotter's product formula.) The kernel of $L_{11, \mu_{0}}$ is spanned by $\left\{\partial_{x_{j}} \varphi_{0}\right\}_{j}$, thanks to the kernel assumption. Thus the kernel of $L_{\mu_{0}}$ is spanned by $\left\{J \varphi_{0}, \partial_{x_{j}} \boldsymbol{\varphi}_{0}, j=1,2,3\right\}$, neither of these functions are $\hat{e}_{3}$-symmetric. Thus, $L_{\mu_{0}}$, as a map $\mathrm{H}_{\hat{e}_{3}}^{1} \subset \mathrm{H}_{\hat{e}_{3}}^{0} \rightarrow \mathrm{H}_{\hat{e}_{3}}^{0}$, is invertible.

We conclude, by the implicit function theorem [6], that there is a neighborhood $W \subset \mathbb{R} \times \mathbb{R}_{+}$with $\left(0, \mu_{0}\right) \in W$ and a unique map $(\tilde{v}, \mu) \mapsto \boldsymbol{\varphi}_{\tilde{v} \hat{e}_{3}, \mu} \in \mathrm{C}^{\infty}\left(W, \mathrm{H}_{\hat{e}_{3}}^{1}\right)$ such that $\boldsymbol{\varphi}_{0 \hat{e}_{3}, \mu_{0}}=\boldsymbol{\varphi}_{0}$ and $\boldsymbol{\varphi}_{\tilde{v} \hat{e}_{3}, \mu}$ solves $F(\tilde{v}, \mu, \boldsymbol{\psi})=0$.

That $\boldsymbol{\varphi}_{\tilde{v} \hat{e}_{3}, \mu} \in \mathrm{H}^{n}$ for any $1 \leq n \in \mathbb{N}$ follows from a simple bootstrap argument; see the proof of Theorem 3 in [9].

Proof of Lemma 4.8. The Hardy-Littlewood-Sobolev inequality together with the Sobolev embedding theorem (see e.g., [21]) shows that $\mathcal{E}_{v, \mu}^{\prime} \in \mathrm{C}\left(\mathrm{H}^{1}, \mathrm{~L}^{2}\right)$. To see that $\mathcal{E}_{\tilde{v} \hat{e}_{3, \mu}}^{\prime}$ preserves $\hat{e}_{3}$-symmetry, let $U$ be either of $K S_{3}$ or $R_{3, \theta}$. Both operations leave $\mathcal{E}_{\tilde{v} \hat{e}_{3}, \mu}$ invariant i.e.,

$$
\mathcal{E}_{\tilde{v} \hat{e}_{3}, \mu}(U \boldsymbol{\psi})=\mathcal{E}_{\tilde{v} \hat{e}_{3}, \mu}(\boldsymbol{\psi}), \quad \boldsymbol{\psi} \in \mathrm{H}^{1}
$$

By (Fréchet) differentiation of (4.10)

$$
\mathcal{E}_{\tilde{v} \hat{e}_{3}, \mu}^{\prime}(U \boldsymbol{\psi})=U \mathcal{E}_{\tilde{v} \hat{e}_{3}, \mu}^{\prime}(\boldsymbol{\psi})
$$


Let $\boldsymbol{\psi}$ satisfy $U \boldsymbol{\psi}=\boldsymbol{\psi}$. Eqn. (4.11) then states

$$
\mathcal{E}_{\tilde{v} \hat{e}_{3}, \mu}^{\prime}(\boldsymbol{\psi})=\mathcal{E}_{\tilde{v} \hat{e}_{3}, \mu}^{\prime}(U \boldsymbol{\psi})=U \mathcal{E}_{\tilde{v} \hat{e}_{3}, \mu}^{\prime}(\boldsymbol{\psi}), \text { where } \boldsymbol{\psi} \in \mathrm{H}^{1}
$$

and hence $\mathcal{E}_{\tilde{v} \hat{e}_{3}, \mu}^{\prime}: \mathrm{H}_{\hat{e}_{3}}^{1} \rightarrow \mathrm{H}_{\hat{e}_{3}}^{0}$. Repeating the argument for higher order derivatives of (4.10) gives that $\mathcal{E}_{\tilde{v} \hat{e}_{3}, \mu}^{(n)}(\boldsymbol{\psi})$ preserves $\hat{e}_{3}$-symmetry.

That $\boldsymbol{\psi} \mapsto \mathcal{E}_{v, \mu}^{\prime}(\boldsymbol{\psi})$ is $\mathrm{C}^{1}$ follows from the Hardy-Sobolev-Littlewood inequality and the Sobolev embedding theorems for $\mathrm{H}^{1}$. That is, let $\boldsymbol{u}, \boldsymbol{w}, \boldsymbol{\xi}, \boldsymbol{\zeta} \in \mathrm{H}^{1}$, then

$$
\begin{aligned}
\sup _{\|\boldsymbol{\xi}\|_{\mathrm{H}^{1}}=1,\|\boldsymbol{\zeta}\|_{\mathrm{H}^{1}}=1}\left|\left(\mathcal{E}_{v, \mu}^{\prime \prime}(\boldsymbol{u}) \boldsymbol{\xi}, \boldsymbol{\zeta}\right)_{2}-\left(\mathcal{E}_{v, \mu}^{\prime \prime}(\boldsymbol{w}) \boldsymbol{\xi}, \boldsymbol{\zeta}\right)_{2}\right| & =\sup _{\|\boldsymbol{\xi}\|_{\mathrm{H}^{1}}=1,\|\boldsymbol{\zeta}\|_{\mathrm{H}^{1}}=1} \\
\left|\left(\frac{1}{|x|} *((\boldsymbol{u}+\boldsymbol{w}) \cdot(\boldsymbol{u}-\boldsymbol{w})), \boldsymbol{\xi} \cdot \boldsymbol{\zeta}\right)_{2}+\left(\boldsymbol{\zeta} \cdot(\boldsymbol{u}-\boldsymbol{w}), \frac{2}{|x|} *(\boldsymbol{u} \cdot \boldsymbol{\xi})\right)_{2}+\left(\boldsymbol{\zeta} \cdot \boldsymbol{w}, \frac{2}{|x|} *((\boldsymbol{u}-\boldsymbol{w}) \cdot \boldsymbol{\xi})\right)_{2}\right| & \leq C\left(\|\boldsymbol{w}\|_{\mathrm{H}^{1}},\|\boldsymbol{u}\|_{\mathrm{H}^{1}}\right)\|\boldsymbol{u}-\boldsymbol{w}\|_{\mathrm{H}^{1}} \cdot \quad(4.13) \\
& \leq C
\end{aligned}
$$

Analogously one can show that $\boldsymbol{\psi} \mapsto \mathcal{E}_{v, \mu}^{\prime}(\boldsymbol{\psi})$ is $\mathrm{C}^{2}$. The polynomial nature of the nonlinearity implies that $\mathcal{E}_{v, \mu}^{(4)}(\boldsymbol{\psi})$ is a (tri-)linear bounded operator independent of $\boldsymbol{\psi}$. Thus $\boldsymbol{\psi} \mapsto \mathcal{E}_{v, \mu}^{\prime}(\boldsymbol{\psi})$ is $\mathrm{C}^{\infty}$ in $\boldsymbol{\psi}$.

The map $(\tilde{v}, \mu) \mapsto \mathcal{E}_{\tilde{v} \hat{e}_{3}, \mu}^{\prime}(\boldsymbol{\psi})$ is linear and hence smooth. Differentiation with respect to either $\mu$ or $\tilde{v}$ does not change $\mathcal{E}_{\tilde{v} \hat{e}_{3}, \mu}^{\prime}(\boldsymbol{\psi})$ 's symmetries.

Proof of Corollary 4.7. The momentum term is the only term in $\mathcal{E}_{v, \mu}$ that breaks the rotation symmetry. For an arbitrary rotation

$$
\mathcal{E}_{v, \mu}(R \psi)=\mathcal{E}_{R^{-1} v, \mu}(\psi)
$$

talking the derivative gives the relation

$$
R^{-1} \mathcal{E}_{v, \mu}^{\prime}(R \psi)=\mathcal{E}_{R^{-1} v, \mu}^{\prime}(\boldsymbol{\psi})
$$

Given the $\hat{e}_{3}$-symmetric function $\boldsymbol{\varphi}_{\tilde{v} \hat{e}_{3}, \mu}$, we find $\boldsymbol{\varphi}_{v, \mu}$ by any rotation, $R$, that takes $\hat{e}_{3}$ to $\hat{v}$ as $\boldsymbol{\varphi}_{v, \mu}=R \boldsymbol{\varphi}_{\tilde{v} \hat{e}_{3}, \mu}$.

This relation between $\boldsymbol{\varphi}_{v, \mu}$ and $\boldsymbol{\varphi}_{\tilde{v} \hat{e}_{3, \mu}, \mu}$ is the key to show the corollary. Let $R_{1}$ be the rotation from $v_{1} \hat{e}_{1}+v_{3} \hat{e}_{3}$ to $|v| \hat{e}_{3}$ given by

$$
R_{1}(\theta):=\left(\begin{array}{ccc}
\cos \theta & 0 & -\sin \theta \\
0 & 0 & 0 \\
\sin \theta & 0 & \cos \theta
\end{array}\right)
$$

where $\theta$ is the angle between $v$ and $\hat{e}_{3}$. We find for $\tilde{v}=|v|>0$ that

$$
\begin{aligned}
\partial_{v_{1}} \boldsymbol{\varphi}_{v, \mu} & =\partial_{v_{1}}\left(R_{1} \boldsymbol{\varphi}_{\tilde{v} \hat{e}_{3}, \mu}\right)=\frac{v_{1}}{\tilde{v}} R_{1} \partial_{\tilde{v}} \boldsymbol{\varphi}_{\tilde{v} \hat{e}_{3}, \mu}+\left(\partial_{v_{1}} \theta\right)\left(\partial_{\theta} R_{1} x\right) \cdot R_{1} \nabla_{x} \boldsymbol{\varphi}_{\tilde{v} \hat{e}_{3}, \mu} \\
& =R_{1}\left(\frac{v_{1}}{\tilde{v}} \partial_{\tilde{v}} \boldsymbol{\varphi}_{\tilde{v} \hat{e}_{3}, \mu}+\left(\partial_{v_{1}} \theta\right)\left(R_{1}^{-1} \partial_{\theta} R_{1} x\right) \cdot \nabla_{x} \boldsymbol{\varphi}_{\tilde{v} \hat{e}_{3}, \mu}\right) .
\end{aligned}
$$


At the point $v_{1}=0, v_{3}=\tilde{v}$ this simplifies to

$$
\left.\partial_{v_{1}} \boldsymbol{\varphi}_{v, \mu}\right|_{v=(0,0, \tilde{v})}=-\frac{1}{\tilde{v}} \hat{e}_{2} \cdot\left(x \wedge \nabla_{x}\right) \boldsymbol{\varphi}_{\tilde{v} \hat{e}_{3}, \mu}
$$

where $\wedge$ is the cross product. The above expression is $\sim J \partial_{x_{1}} \boldsymbol{\varphi}_{\tilde{v} \hat{e}_{3}, \mu}$. Analogously for $v=\left(0, v_{2}, v_{3}\right)$ we find

$$
\left.\partial_{v_{2}} \boldsymbol{\varphi}_{v, \mu}\right|_{v=(0,0, \tilde{v})}=\frac{1}{\tilde{v}} \hat{e}_{1} \cdot\left(x \wedge \nabla_{x}\right) \boldsymbol{\varphi}_{\tilde{v} \hat{e}_{3}, \mu} \sim J \partial_{x_{2}} \boldsymbol{\varphi}_{\tilde{v} \hat{e}_{3}, \mu}
$$

Recall from Proposition 4.5 that $\partial_{\tilde{v}} \boldsymbol{\varphi}_{v, \mu} \in \mathrm{H}_{\hat{e}_{3}}^{1}$ and thus $\sim J \partial_{x_{3}} \boldsymbol{\varphi}_{\tilde{v} \hat{e}_{3}, \mu}$. Therefore we have shown the corollary for a given coordinate system, i.e., coordinates such that $\boldsymbol{\varphi}_{v, \mu}$ is $\boldsymbol{\varphi}_{\tilde{v} \hat{e}_{3}, \mu}$, rotation $R$, once again, from $\hat{e}_{3}$ to $\hat{v}$ of this case gives the general result.

\subsection{Proof of exponential decay of tangent vectors}

In this subsection we prove Proposition 4.3(iii), i.e., the pointwise exponential decay of the tangent vectors $\left\{\partial_{x_{j}} \boldsymbol{\varphi}_{v, \mu}, \partial_{v_{j}} \boldsymbol{\varphi}_{v, \mu}, J \boldsymbol{\varphi}_{v, \mu}, \partial_{\mu} \boldsymbol{\varphi}_{v, \mu}\right\}$. In [9, App. C] we showed that $\boldsymbol{\varphi}_{v, \mu}$ satisfies the bound

$$
\left|\boldsymbol{\varphi}_{v, \mu}\right| \leq c_{1}(\beta) \mathrm{e}^{-\beta|x|},
$$

for $0<\beta<\min \left(m,\left(\mu-\mu_{l}\right)\left(1-v^{2}\right)^{-1 / 2}\right)$, where $\mu_{l}$ is defined in Proposition 4.3. The remaining tangent vectors all satisfy an equation of the type

$$
\boldsymbol{u}=F(\boldsymbol{u}, \boldsymbol{w})
$$

with

$$
F(\boldsymbol{u}, \boldsymbol{w}):=\left(H_{v}+\mu\right)^{-1}\left(W_{1} \boldsymbol{u}+W_{2}(\boldsymbol{u})+\boldsymbol{w}\right)
$$

and

$$
H_{v}=\sqrt{-\Delta+m^{2}}-m-J v \cdot \nabla, W_{1}:=\frac{1}{|x|} *\left|\boldsymbol{\varphi}_{v, \mu}\right|^{2}, W_{2}(\boldsymbol{u}):=\left(\frac{2}{|x|} *\left(\boldsymbol{u} \cdot \boldsymbol{\varphi}_{v, \mu}\right)\right) \boldsymbol{\varphi}_{v, \mu},
$$

for $\mu>\mu_{l}$ and some $\boldsymbol{w}$ depending on the tangent vector, see (4.4)-(4.5).

We have the following result.

Lemma 4.9. Let $m>0, \mu>\mu_{l}$ and let $\boldsymbol{u}$ be a solution to (4.22), for some $\boldsymbol{w}$ with $|\boldsymbol{w}| \leq c_{2} \mathrm{e}^{-\beta_{2}|x|}$ where $c_{2}, \beta_{2}$ are some positive constants. Then, there is $\theta>0$ and a constant $C(\theta)>0$ such that

$$
|\boldsymbol{u}| \leq C(\theta) \mathrm{e}^{-\theta|x|}
$$

We now return to equations (4.4)-(4.5) to find pairs $(\boldsymbol{u}, \boldsymbol{w})$ that solve (4.22). These are $\left(\partial_{x_{j}} \boldsymbol{\varphi}_{v, \mu}, 0\right),\left(\partial_{\mu} \boldsymbol{\varphi}_{v, \mu},-\boldsymbol{\varphi}_{v, \mu}\right)$ and $\left(\partial_{v_{j}} \boldsymbol{\varphi}_{v, \mu}, J \partial_{x_{j}} \boldsymbol{\varphi}_{v, \mu}\right)$. The first pair, inserted in Lemma 4.9 ensures that $\partial_{x_{j}} \boldsymbol{\varphi}_{v, \mu}$ is pointwise exponentially decaying. Thus, we know that the last two pairs also satisfy the assumptions of Lemma 4.9 and hence, both $\partial_{\mu} \boldsymbol{\varphi}_{v, \mu}$ and $\partial_{v_{j}} \boldsymbol{\varphi}_{v, \mu}$ are pointwise exponentially decaying. It remains to prove Lemma 4.9. 
Proof of Lemma 4.9. The proof is based on [28] as presented in [15] and we extend the result to include the source terms. That the integral kernel of $\left(H_{v}+\mu\right)^{-1}, G_{\mu, v}$, satisfies the bound, [9, App. C]

$$
\left|G_{\mu, v}(x)\right| \leq c_{3} \frac{\mathrm{e}^{-\delta|x|}}{|x|^{2}}
$$

for some $\delta>0$ depending on $m>0,|v|<1$, and $\mu>\mu_{l}$.

Our first goal is to use (4.22) to bound $|\boldsymbol{u}|$ as

$$
|\boldsymbol{u}(x)| \leq h_{\theta}(x) M(x)+C_{1} \mathrm{e}^{-\gamma|x|},
$$

where $\theta>0$ remains to be chosen later, and

$$
M(x):=\sup _{x^{\prime}}\left|\boldsymbol{u}\left(x^{\prime}\right)\right| \mathrm{e}^{-\theta\left|x-x^{\prime}\right|}
$$

To this end, we need estimates on each term in (4.23) and we begin with the $W_{2}(\boldsymbol{u})$ term

$$
W_{2}(\boldsymbol{u})\left(x^{\prime}\right)=\boldsymbol{\varphi}_{v, \mu}\left(x^{\prime}\right) \int_{\mathbb{R}^{3}} \frac{1}{\left|x^{\prime}-y\right|} \boldsymbol{u}(y) \cdot \boldsymbol{\varphi}_{v, \mu}(y) \mathrm{d} y
$$

The identity $\boldsymbol{u}(y)=\boldsymbol{u}(y) \mathrm{e}^{-\theta|y-x|} \mathrm{e}^{\theta|y-x|}$, the inequality $|y-x| \leq\left|y-x^{\prime}\right|+\left|x^{\prime}-x\right|$, and the upper bound (4.21) lead to

$$
\left|W_{2}(\boldsymbol{u})\left(x^{\prime}\right)\right| \leq C_{2}^{\prime} M(x) \mathrm{e}^{-\beta\left|x^{\prime}\right|+\theta\left|x-x^{\prime}\right|} \int_{\mathbb{R}^{3}} \frac{\mathrm{e}^{\theta\left|x^{\prime}-y\right|}}{\left|x^{\prime}-y\right|} \mathrm{e}^{-\beta|y|} \mathrm{d} y .
$$

We evaluate the integral, with $0<\theta<\beta$, to find for some $C_{2}=C_{2}(\theta)>0$ that

$$
\left|W_{2}(\boldsymbol{u})\left(x^{\prime}\right)\right| \leq C_{2} \frac{\mathrm{e}^{\theta\left|x-x^{\prime}\right|}}{1+\left|x^{\prime}\right|} M(x)
$$

The estimate for $W_{1}$ follows similarly, by once again integrating an integral of the type that appears in (4.30):

$$
\left|W_{1}\left(x^{\prime}\right)\right| \leq C_{3} \frac{1}{1+\left|x^{\prime}\right|} .
$$

The 'potentials' $W_{1}$ and $W_{2}$ are hence bounded and decaying and we can choose $h_{\theta}$ to be

$$
h_{\theta}(x):=C_{4} \int_{\mathbb{R}^{3}} \frac{\mathrm{e}^{-(\delta-\theta)\left|x-x^{\prime}\right|}}{\left|x-x^{\prime}\right|^{2}} \frac{1}{1+\left|x^{\prime}\right|} \mathrm{d} x^{\prime},
$$

where $C_{4}$ is composed of the constants $c_{3}, C_{2}$ and $C_{3}$.

We use the integral kernel $G_{\mu, v}$ of $\left(H_{v}+\mu\right)^{-1}$ to express the last term of (4.23). By (4.26), we have

$$
\left|G_{\mu, v} * \boldsymbol{w}\right| \leq c_{3} \int_{\mathbb{R}^{3}} \frac{\mathrm{e}^{-\delta\left|x^{\prime}\right|}}{\left|x^{\prime}\right|^{2}}\left|\boldsymbol{w}\left(x-x^{\prime}\right)\right| \mathrm{d} x^{\prime} .
$$


The assumed, pointwise exponential decay of $\boldsymbol{w}$ together with the inequality $\left|x-x^{\prime}\right| \geq$ ||$x|-| x^{\prime}||$ yields

$$
\left|G_{\mu, v} * \boldsymbol{w}\right| \leq C_{1} \mathrm{e}^{-\gamma|x|},
$$

where $\gamma=\min \left(\delta, \beta_{2}\right)$ and $C_{1}>0$ are suitable constants. We have thus established (4.27).

To proceed, we show that $h_{\theta}$ is bounded and that it decays pointwise as $|x| \rightarrow \infty$. The first of these properties follows from Young's inequality, since for $\theta<\delta, \mathrm{e}^{\theta|\cdot|} G_{\mu, v}(\cdot) \in \mathrm{L}^{1}$ and $(1+|\cdot|)^{-1}<1 \in \mathrm{L}^{\infty}$

$$
\sup _{x}\left|h_{\theta}\right| \leq C_{4}\left\|\mathrm{e}^{\theta|\cdot|} G_{\mu, v}(\cdot)\right\|_{1} \sup _{x}\left|(1+|x|)^{-1}\right|=C_{5}(\theta)<\infty .
$$

To show the decay of $h_{\theta}$ as $|x| \rightarrow \infty$, let $\alpha:=\delta-\theta, \alpha>0$, we use (4.33) and split the region of integration into two parts $\left|x-x^{\prime}\right| \leq \kappa,\left|x-x^{\prime}\right|>\kappa$. In the outer region we use the uniform bound of $\left(1+\left|x^{\prime}\right|\right)^{-1}<1$ to find

$$
\int_{\left|x-x^{\prime}\right|>\kappa} \frac{\mathrm{e}^{-\alpha\left|x-x^{\prime}\right|}}{\left|x-x^{\prime}\right|^{2}} \frac{1}{1+\left|x^{\prime}\right|} \mathrm{d} x^{\prime} \leq \frac{1}{\kappa^{2}} \int_{\mathbb{R}^{3}} \mathrm{e}^{-\alpha\left|x^{\prime}\right|} \mathrm{d} x^{\prime},
$$

and in the inner region,

$$
\int_{\left|x-x^{\prime}\right| \leq \kappa} \frac{\mathrm{e}^{-\alpha\left|x-x^{\prime}\right|}}{\left|x-x^{\prime}\right|^{2}} \frac{1}{1+\left|x^{\prime}\right|} \mathrm{d} x^{\prime} \leq \int_{|y| \leq \kappa} \frac{\mathrm{d} y}{|y|^{2}(1+|x-y|)} \leq \frac{4 \pi \kappa}{1+|| x|-\kappa|},
$$

The choice of $\kappa=|x|^{1 / 2}$ ensures that $h_{\theta} \leq C_{6}|x|^{-1 / 2}$ as $|x| \rightarrow \infty$ and $\theta \leq \delta$.

The following two identities will be used repeatedly in the next step of the proof, let $\theta>0, \gamma>0$

$$
\sup _{y} \mathrm{e}^{-\theta|x-y|-\theta\left|y-x^{\prime}\right|}=\mathrm{e}^{-\theta\left|x-x^{\prime}\right|}, \sup _{y} \mathrm{e}^{-\theta|y|-\gamma|y-x|}=\mathrm{e}^{-\min (\theta, \gamma)|x|} .
$$

The exponential decay of $\boldsymbol{u}$ now follows from the properties of $h_{\theta}$, through two inequalities. Since $h_{\theta}$ decay, for a fixed small $\theta<\delta$, there is a radius $R$, such that for $|x|>R$ we have that $h_{\theta} \leq C_{6} R^{-1 / 2}$. In this exterior region, we use (4.27) together with (4.39) to obtain

$$
\begin{aligned}
\sup _{\left|x^{\prime}\right|>R}\left|\boldsymbol{u}\left(x^{\prime}\right)\right| \mathrm{e}^{-\theta\left|x-x^{\prime}\right|} \leq \sup _{\left|x^{\prime}\right|>R}\left(h_{\theta}\left(x^{\prime}\right) M\left(x^{\prime}\right)+C_{1} \mathrm{e}^{-\gamma\left|x^{\prime}\right|}\right) \mathrm{e}^{-\theta\left|x-x^{\prime}\right|} & \\
& \leq C_{6} R^{-1 / 2} M(x)+\sup _{\left|x^{\prime}\right|>R} C_{1} \mathrm{e}^{-\gamma\left|x^{\prime}\right|-\theta\left|x-x^{\prime}\right|}
\end{aligned}
$$

In the interior we have

$$
\begin{aligned}
& \sup _{\left|x^{\prime}\right| \leq R}\left|\boldsymbol{u}\left(x^{\prime}\right)\right| \mathrm{e}^{-\theta\left|x-x^{\prime}\right|} \leq \\
& \sup _{\left|x^{\prime}\right| \leq R}\left(h_{\theta}\left(x^{\prime}\right)\left(\sup _{|y| \leq R}|\boldsymbol{u}(y)| \mathrm{e}^{-\theta\left|x^{\prime}-y\right|}+\sup _{|y|>R}|\boldsymbol{u}(y)| \mathrm{e}^{-\theta\left|x^{\prime}-y\right|}\right)+C_{1} \mathrm{e}^{-\gamma\left|x^{\prime}\right|}\right) \mathrm{e}^{-\theta\left|x-x^{\prime}\right|} .
\end{aligned}
$$


Insert the result (4.40) into (4.41). The upper bound (4.36) ensures that $h_{\theta}<C_{5}$; the exterior term $\sup _{|y| \geq R}|\boldsymbol{u}(y)| \mathrm{e}^{-\theta\left|x^{\prime}-y\right|}$ is estimated by (4.40); for the interior term $\sup _{|y| \leq R}|\boldsymbol{u}(y)| \mathrm{e}^{-\theta\left|x^{\prime}-y\right|}$ we have by continuity and boundedness of $\boldsymbol{u}$ that $|\boldsymbol{u}| \leq C_{7}^{\prime}(R)$ and hence $\sup _{|y| \leq R}|\boldsymbol{u}(y)| \mathrm{e}^{-\theta\left|x^{\prime}-y\right|} \leq C_{7}(R, \theta) \mathrm{e}^{-\theta\left|x^{\prime}\right|} ;$ yields (4.41) to become

$$
\begin{aligned}
& \sup _{\left|x^{\prime}\right| \leq R}\left|\boldsymbol{u}\left(x^{\prime}\right)\right| \mathrm{e}^{-\theta\left|x-x^{\prime}\right|} \leq C_{1} \sup _{\left|x^{\prime}\right| \leq R} \mathrm{e}^{-\gamma\left|x^{\prime}\right|} \mathrm{e}^{-\theta\left|x-x^{\prime}\right|} \\
& \quad+C_{5} \sup _{\left|x^{\prime}\right| \leq R}\left(C_{7} \mathrm{e}^{-\theta\left|x^{\prime}\right|}+C_{6} R^{-1 / 2} M\left(x^{\prime}\right)+C_{1} \sup _{|y|>R} \mathrm{e}^{-\gamma|y|-\theta|x-y|}\right) \mathrm{e}^{-\theta\left|x-x^{\prime}\right|} .
\end{aligned}
$$

Adding (4.40) to (4.42), rewriting and the use of (4.39) give

$$
M(x) \leq C_{8}\left(R^{-1 / 2} M(x)+\mathrm{e}^{-\min (\gamma, \theta)|x|}\right)+C_{9}(R, \theta) \mathrm{e}^{-\theta|x|},
$$

for suitable constants $C_{8}=C_{8}(\theta), C_{9}$. By choice of $R=R^{*}$, sufficiently large and $\theta>0$, sufficiently small, we find

$$
M(x) \leq C^{\prime}\left(R^{*}, \theta\right) \mathrm{e}^{-\theta|x|},
$$

This upper bound inserted into (4.27) together with (4.36) yields $|\boldsymbol{u}| \leq C(\theta) \mathrm{e}^{-\theta|x|}$ and we have proved the lemma.

\subsection{Proof of the Stability Condition}

We now derive the "stability condition" stated in Proposition 4.3(iv) for unboosted ground states, $\boldsymbol{\varphi}_{v=0, \mu}(x)=\left(\varphi_{\mu}, 0\right)$. As mentioned in Remark 4.6 (see also [23, 9]), these functions can be assumed to be real-valued and spherically symmetric. In view of this, we introduce the subspace

$$
\mathrm{H}_{\mathrm{rad}}^{s}\left(\mathbb{R}^{3}, \mathbb{R}\right)=\left\{\psi \in \mathrm{H}^{s}\left(\mathbb{R}^{3} ; \mathbb{R}\right): \psi \text { is spherically symmetric }\right\}
$$

for $s \geq 0$.

Lemma 4.10. Suppose that Assumption 2.1 holds. Then, for almost every $0<N<N_{\mathrm{c}}$, there exists an unboosted ground state, $\varphi_{*}=\varphi_{v=0, \mu_{*}}$, with $\mathcal{N}\left(\left(\varphi_{*}, 0\right)\right)=N$ and Lagrange multiplier, $\mu_{*}$, satisfying the following properties. For every sufficiently small $\delta>0$, there exists a $\mathrm{C}^{\infty}$-map

$$
\left(\mu_{*}-\delta, \mu_{*}+\delta\right) \rightarrow \mathrm{H}_{\mathrm{rad}}^{1}\left(\mathbb{R}^{3}, \mathbb{R}\right), \quad \mu \mapsto \varphi_{\mu},
$$

where $\left(\varphi_{\mu}, 0\right)$ solves (2.19) with $v=0$ and we have that $\varphi_{\mu_{*}}=\varphi_{*}$. In addition, there exists a non-empty interval $I \subset\left(\mu_{*}-\delta, \mu_{*}+\delta\right)$, such that

$$
\frac{\mathrm{d}}{\mathrm{d} \mu} \mathcal{N}\left(\left(\varphi_{\mu}, 0\right)\right)>0
$$

holds for all $\mu \in I$. 
Proof of Lemma 4.10. By Remark 4.6(b), we can assume that unboosted ground states $\varphi(x)=\varphi_{v=0, \mu}$ are spherical symmetric and real-valued. Let $E(N):=\inf \left\{\mathcal{E}_{0,0}(\boldsymbol{\psi}): \boldsymbol{\psi} \in\right.$ $\left.\mathrm{H}^{\frac{1}{2}}, \mathcal{N}(\boldsymbol{\psi})=N\right\}$. It is known that the function $E:\left(0, N_{\mathrm{c}}\right) \rightarrow \mathbb{R}$ is strictly concave $[9$, Lemma 2.3]. This implies in particular the following properties.

- $E(N)$ is continuous on $\left(0, N_{\mathrm{c}}\right)$.

- $E_{-}^{\prime}(N)$ and $E_{+}^{\prime}(N)$ (which denote the left and right derivative, respectively) exist for all $N \in\left(0, N_{\mathrm{c}}\right)$.

- $E^{\prime}(N)=E_{-}^{\prime}(N)=E_{+}^{\prime}(N)$ for all $N \in\left(0, N_{\mathrm{c}}\right) \backslash \Sigma$, where $\Sigma$ is some countable set.

For convenience, we denote the set where $E^{\prime}(N)$ exists by

$$
\Sigma^{c}:=\left(0, N_{\mathrm{c}}\right) \backslash \Sigma \text {. }
$$

Let us now pick $N_{*} \in \Sigma^{c}$ and a strictly decreasing sequence, $\left(N_{k}\right)$, in $\Sigma^{c}$ such that

$$
N_{k} \searrow N_{*}, \quad \text { as } k \rightarrow \infty \text {. }
$$

By density $\Sigma^{c} \subset\left(0, N_{\mathrm{c}}\right)$, this always possible. Correspondingly, let $\left(\varphi_{k}\right) \subset \mathrm{H}^{\frac{1}{2}}$ be a sequence of minimizers with $\mathcal{E}_{0,0}\left(\left(\varphi_{k}, 0\right)\right)=E\left(N_{k}\right)$ and $\mathcal{N}\left(\left(\varphi_{k}, 0\right)\right)=N_{k}$, which, by continuity of $E(N)$, implies that

$$
\mathcal{E}_{0,0}\left(\left(\varphi_{k}, 0\right)\right) \rightarrow E\left(N_{*}\right) \quad \text { and } \quad \mathcal{N}\left(\left(\varphi_{k}, 0\right)\right) \rightarrow N_{*}, \quad \text { as } k \rightarrow \infty .
$$

By arguments similar to those in the proof of [9, Theorem 2] and the relative compactness property stated in $\left[9\right.$, Theorem 1], we see that $\left(\varphi_{k}\right)$, after passing to a subsequence, converges strongly in $\mathrm{H}_{\text {rad }}^{\frac{1}{2}}$ to some minimizer $\varphi_{*}$ with $\mathcal{N}\left(\left(\varphi_{*}, 0\right)\right)=N_{*}$ and Lagrange multiplier $-\mu_{*}$. (Note that due to $v=0$, we can restrict our attention to radial functions and translations do not have to be taken into account.)

Next, we observe that any $\varphi_{k}$ satisfies the identity

$$
E\left(N_{k}\right)-\frac{1}{4} \int_{\mathbb{R}^{3}}\left(\frac{1}{|x|} *\left|\varphi_{n_{k}}\right|^{2}\right)\left|\varphi_{n_{k}}\right|^{2} \mathrm{~d} x=-\mu_{k} N_{k}
$$

where $-\mu_{k}$ is the Lagrange multiplier for the minimizer $\varphi_{k}$. This identity follows from multiplication of the Euler-Lagrange equation (2.19) with $\left(\varphi_{k}(x), 0\right)$ and integration. Now we claim that

$$
E^{\prime}\left(N_{k}\right)=-\mu_{k}
$$

holds for all $k$. Note that $E^{\prime}\left(N_{k}\right)$ exists due to $N_{k} \in \Sigma^{c}$ for all $k$. To prove (4.52), we observe that $\mathcal{E}_{0,0}\left(\sqrt{\tau}\left(\varphi_{k}, 0\right)\right) \geq E\left(\tau N_{k}\right)$ holds for all $\tau \geq 0$ with equality for $\tau=1$. Hence it is straightforward to see that the right derivative, $E_{+}^{\prime}\left(N_{k}\right)$, obeys the following estimate

$$
\begin{aligned}
E_{+}^{\prime}\left(N_{k}\right) & =\frac{1}{N_{k}} \lim _{N \searrow N_{k}} \frac{E(N)-E\left(N_{k}\right)}{N / N_{k}-1} \leq \frac{1}{N_{k}} \lim _{\tau \searrow 1} \frac{\mathcal{E}_{0,0}\left(\sqrt{\tau}\left(\varphi_{k}, 0\right)\right)-\mathcal{E}_{0,0}\left(\left(\varphi_{k}, 0\right)\right)}{\tau-1} \\
& =\frac{1}{N_{k}} \lim _{\tau \searrow 1} \frac{d}{d \tau} \mathcal{E}_{0,0}\left(\sqrt{\tau}\left(\varphi_{k}, 0\right)\right)=\left.\frac{1}{2 N_{l} \sqrt{\tau}}\right|_{\tau=1}\left(\mathcal{E}_{0,0}^{\prime}\left(\left(\varphi_{k}, 0\right)\right),\left(\varphi_{k}, 0\right)\right)_{2} \\
& =\frac{-\mu_{k}}{N_{k}} \frac{1}{2} \int_{\mathbb{R}^{3}}\left|\varphi_{k}\right|^{2} \mathrm{~d} x=-\mu_{k},
\end{aligned}
$$


using the Euler-Lagrange equation $\mathcal{E}_{0,0}^{\prime}\left(\left(\varphi_{k}, 0\right)\right)=-\mu_{k}\left(\varphi_{k}, 0\right)$. Similarly, we obtain $-\mu_{k} \leq E_{-}^{\prime}\left(N_{k}\right)$. Since $E^{\prime}\left(N_{k}\right)$ exists for $N_{k} \in \Sigma^{c}$, we have equality and we conclude that (4.52) holds.

Next, let us define the map

$$
G(\psi, \mu):=\left(\sqrt{-\Delta+m^{2}}-m\right) \psi-\left(\frac{1}{|x|} *|\psi|^{2}\right) \psi+\mu \psi,
$$

which is seen to a $\mathrm{C}^{\infty}$-map $G: \mathrm{H}_{\text {rad }}^{1} \times \mathbb{R} \rightarrow \mathrm{L}_{\text {rad }}^{2}$, see the proof of Proposition 4.5 and Remark 4.6(b). Moreover, we note that $G\left(\varphi_{*}, \mu_{*}\right)=0$ holds and we have that $\partial_{\psi} G\left(\varphi_{*}, \mu_{*}\right)$ equals $L_{11, \mu_{*}}$ restricted on $\mathrm{H}_{\mathrm{rad}}^{1}$. But Assumption 2.1 implies that $L_{11, \mu_{*}}$ restricted to $\mathrm{H}_{\mathrm{rad}}^{1}$ has trivial kernel (since $\partial_{x_{i}} \phi_{*} \notin \mathrm{H}_{\mathrm{rad}}^{1}$ ). Thus, we can apply the implicit function theorem to find a unique $\mathrm{C}^{\infty}$-map

$$
\left(\mu_{*}-\delta, \mu_{*}+\delta\right) \longrightarrow U, \quad \mu \longmapsto \varphi_{\mu}
$$

for every sufficiently small $\delta>0$, where $\varphi_{\mu_{*}}=\varphi_{*}$ and $U$ is some open $\mathrm{H}_{\mathrm{rad}}^{1}$-neighborhood around $\varphi_{*}$.

We now show that strong convergence of $\varphi_{k}$ to $\varphi_{*}$ in $\mathrm{H}_{\text {rad }}^{\frac{1}{2}}$ implies strong convergence in $\mathrm{H}_{\mathrm{rad}}^{1}$. This can be seen as follows. Each $\varphi_{k}$ satisfies the equation

$$
\varphi_{k}=R_{\mu_{k}} F\left(\varphi_{k}\right)
$$

where $R_{\mu}:=\left(H_{0}+\mu\right)^{-1}$ with $H_{0}:=\sqrt{-\Delta+m^{2}}-m$, and $F(\varphi):=\left(|x|^{-1} *|\varphi|^{2}\right) \varphi$. Therefore we have

$$
\begin{aligned}
\left\|\varphi_{k}-\varphi_{*}\right\|_{\mathrm{H}^{1}}= & \left\|R_{\mu_{k}} F\left(\varphi_{k}\right)-R_{\mu_{*}} F\left(\varphi_{*}\right)\right\|_{\mathrm{H}^{1}} \\
\leq & \left\|\left(R_{\mu_{k}}-R_{\mu_{*}}\right)\left(F\left(\varphi_{k}\right)+F\left(\varphi_{*}\right)\right)\right\|_{\mathrm{H}^{1}} \\
& +\left\|\left(R_{\mu_{k}}+R_{\mu_{*}}\right)\left(F\left(\varphi_{k}\right)-F\left(\varphi_{*}\right)\right)\right\|_{\mathrm{H}^{1}} .
\end{aligned}
$$

By (4.51), the fact that $\varphi_{k} \rightarrow \varphi_{*}$ in $\mathrm{H}_{\text {rad }}^{\frac{1}{2}}$, and $N_{k} \searrow N_{*}$, we see that $\mu_{k} \searrow \mu_{*}$ (note that (4.52) holds and that $E^{\prime}(N)$ is strictly decreasing on $\Sigma^{c}$ ). Using now the resolvent identity $R_{\mu_{k}}-R_{\mu_{*}}=\left(\mu_{*}-\mu_{k}\right) R_{\mu_{k}} R_{\mu_{*}}$, as well as $\left\|R_{\mu_{k}}\right\|_{\mathrm{L}^{2} \rightarrow \mathrm{H}^{1}} \leq C / \mu_{k}$, we deduce that

$$
\left\|\varphi_{k}-\varphi_{*}\right\|_{\mathrm{H}^{1}} \leq C\left(\left|\mu_{k}-\mu_{*}\right|+\left\|\varphi_{k}-\varphi_{*}\right\|_{\mathrm{H}^{\frac{1}{2}}}\right) \rightarrow 0 \quad \text { as } k \rightarrow \infty,
$$

where we also used the local Lipschitz estimate

$$
\|F(u)-F(v)\|_{2} \leq\|F(u)-F(v)\|_{\mathrm{H}^{\frac{1}{2}}} \leq C\left(\|u\|_{\mathrm{H}^{\frac{1}{2}}}^{2}+\|v\|_{\mathrm{H}^{\frac{1}{2}}}^{2}\right)\|u-v\|_{\mathrm{H}^{\frac{1}{2}}},
$$

see $\left[20, \S 3\right.$ Lemma 1]. In estimate (4.59), $C=C\left(M, \mu_{*}\right)$ denotes a suitable constant with $M=\sup _{k}\left\|\varphi_{k}\right\|_{\mathrm{H}^{\frac{1}{2}}}$.

By the strong convergence of $\varphi_{k}$ to $\varphi_{*}$ in $\mathrm{H}_{\mathrm{rad}}^{1}$ shown above, we thus obtain that $\varphi_{k} \in U$ whenever $k \geq k_{0}$, where $k_{0}$ is sufficiently large. Moreover, since the left-hand side of (4.51) converges to its value at $N_{*}$, we conclude that $\mu_{k}$ converges to $\mu_{*}$. In addition, by (4.52) and the strict concavity, we deduce that $\mu_{k} \searrow \mu_{*}$ (note $E^{\prime}(N)$ has to 
be strictly decreasing on $\left.\Sigma^{c}\right)$. In summary, we find that $\varphi_{k_{0}} \in U$ and $\mu_{k_{0}} \in\left(\mu_{*}-\delta, \mu_{*}+\delta\right)$ for some $k_{0}$ and $\mu_{k_{0}}>\mu_{*}$. By uniqueness of the map (4.56), we see that $\varphi_{k_{0}}=\varphi_{\mu_{k_{0}}}$, where $\varphi_{k_{0}}$ belongs to the sequence $\left(\varphi_{k}\right)$ and $\varphi_{\mu_{k_{0}}}$ is constructed via the map (4.56). Hence we have that the $\mathrm{C}^{\infty}$-function

$$
f(\mu):=\mathcal{N}\left(\varphi_{\mu}\right)
$$

satisfies $f\left(\mu_{*}\right)<f\left(\mu_{k_{0}}\right)$. By the mean-value theorem, there exists some $\xi \in\left(\mu_{*}-\delta, \mu_{*}+\delta\right)$ such that $f^{\prime}(\xi)>0$. By continuity of $f^{\prime}$, we conclude that $f^{\prime}(\mu)>0$ for all $\mu \in I$ with some open interval $I$ containing $\xi$. This completes the proof of Lemma 4.10.

\subsection{Completion of the proof of Proposition 4.3}

In Appendix A we prove Proposition 4.3(v). Each part is shown for some small open neighborhood in $\mathbb{R}^{2}$ around the point $\left(\mu_{0}, 0\right)$. We can now complete the proof of Proposition 4.3 .

Proof of Proposition 4.3. Let $\mu_{0}>0$, with minimizer, $\boldsymbol{\varphi}_{\mu_{0}}$, be a point where Assumption 2.1 holds. Above, in Sec. 4.3, we showed that for almost all $N>0$ with corresponding $\mu_{*}$ there is an open non-empty interval $I$ around $\mu_{*}$ and a unique spherically symmetric, real function $\boldsymbol{\varphi}_{\mu}$ such that $\partial_{\mu} \mathcal{N}\left(\boldsymbol{\varphi}_{\mu}\right) \geq c$, and that $c$ is independent of $\mu$. We thus have singled out an 'admissible' $\varphi_{\mu_{*}}$ around which we construct our ground states.

In Sec. 4.1 we constructed $\varphi_{v, \mu}$ and its symmetries around $\boldsymbol{\varphi}_{\mu_{*}}$ for $(\tilde{v}, \mu) \subset W$, where $W$ is some open, non-empty neighborhood in $\mathbb{R}^{2}$ and $v=\tilde{v} \hat{v}$, and $\hat{v}=v /|v|$. That $\mu_{*}>0$ and $\mu_{l}(0)=0$ ensure the existence of a, possibly smaller, open non-empty set, also denoted $W$ with points so that $\mu>\mu_{|v|}$ is satisfied. Thus for all $(\tilde{v}, \mu) \in W$ we have shown Proposition 4.3(i), (ii).

The proof of the exponential decay (iii) (Sec. 4.2) does not constraint further the set $W$.

We now show Proposition 4.3(iv), i.e., the stability condition for non-zero velocities. At $v=0$ the stability condition holds, and since the above constructed $\varphi_{v, \mu}$ depends continuously on $\tilde{v}$ and $\mu$ so does $\mathcal{N}\left(\boldsymbol{\varphi}_{v, \mu}\right)$ and there exists an open, non-empty, possibly smaller, region $W_{1} \subset W$ such that $\partial_{\mu} \mathcal{N}\left(\boldsymbol{\varphi}_{v, \mu}\right)>c / 2$ for all $(\tilde{v}, \mu) \in W_{1}$.

The spectral properties of $L_{v, \mu}$, Proposition 4.3(v) (see Appendix A) is shown for $(\tilde{v}, \mu) \in W_{2}$, where $W_{2}$ is some open non-empty neighborhood around $\left(0, \mu_{*}\right)$ such that $W_{2} \subset W_{1}$.

Finally, we have this small open and non-empty set $W_{2}$, where (i)-(v) hold, we now choose $r_{0}>0$, and an open non-empty interval $I_{0}$ such that $W_{3}:=\left(-r_{0}, r_{0}\right) \times I_{0}$ and $W_{3} \Subset W_{2}$. This concludes the proof of Proposition 4.3. 


\section{The symplectic form reduced to the soliton man- ifold}

The purpose of this section is to show that the symplectic form $\omega(\cdot, \cdot)$ reduced to a subset of the soliton manifold $\mathrm{M}_{0}=\mathrm{M}\left(\mathrm{Z}_{0}\right)$ is non-degenerate. The result follows if the matrix $\left(\Omega_{\boldsymbol{\varphi}_{\zeta}}\right)_{j k}:=\omega\left(\boldsymbol{z}_{j, \zeta}, \boldsymbol{z}_{k, \zeta}\right)$ is invertible. Here $\boldsymbol{z}_{j, \zeta}$ are elements in the tangent space $\mathrm{T}_{\boldsymbol{\varphi}_{\zeta}} \mathrm{M}$, for some $\mathrm{M} \subset \mathrm{M}_{0}$, defined by (see also (3.6))

$$
\left\{\boldsymbol{z}_{1, \zeta}, \ldots, \boldsymbol{z}_{8, \zeta}\right\}:=\left\{\partial_{x_{1}} \boldsymbol{\varphi}_{\zeta}, \partial_{x_{2}} \boldsymbol{\varphi}_{\zeta}, \partial_{x_{3}} \boldsymbol{\varphi}_{\zeta}, \partial_{v_{1}} \boldsymbol{\varphi}_{\zeta}, \partial_{v_{2}} \boldsymbol{\varphi}_{\zeta}, \partial_{v_{3}} \boldsymbol{\varphi}_{\zeta}, \partial_{\vartheta} \boldsymbol{\varphi}_{\zeta}, \partial_{\mu} \boldsymbol{\varphi}_{\zeta}\right\}
$$

Proposition 5.1. Let $\mathrm{Z}(r, I)$ be as in (3.2) and let $\Omega_{\boldsymbol{\varphi}_{\zeta}}$ be defined as above. Under Assumption 2.1, there are numbers $0<r_{1}<r_{0}, \kappa>0$ and an open non-empty interval $I_{1} \Subset I_{0}$ such that

$$
\operatorname{det} \Omega_{\varphi_{\zeta}} \geq \kappa>0 \text {. }
$$

for all $\zeta \in \mathrm{Z}\left(r_{1}, I_{1}\right)$. The constant $\kappa$ depends only on $r_{1}$ and $I_{1}$.

Using the definitions (3.2) and (3.3) we set $\mathrm{Z}_{1}:=\mathrm{Z}\left(r_{1}, I_{1}\right)$ and $\mathrm{M}_{1}:=\mathrm{M}\left(\mathrm{Z}_{1}\right)$. We have now defined the first number and non-empty interval in the sequence mentioned in the main theorem. The size of $\left|\Omega_{\varphi_{\zeta}}^{-1}\right|$ may depend on $r_{1}$ and $I_{1}$, thus the first requirement on $\varepsilon$ is that $\left|\Omega_{\boldsymbol{\varphi}_{\zeta}}^{-1}\right|=\mathcal{O}(1)$. This is a natural requirement, as we will see in Proposition 7.1. As a direct consequence of the non-degeneracy of $\Omega_{\boldsymbol{\varphi}_{\zeta}}$ we have the following corollary.

Corollary 5.2. For all $0 \neq \boldsymbol{z} \in \mathrm{T}_{\boldsymbol{\varphi}_{\zeta}} \mathrm{M}_{1}$, there is at least one element, $\tilde{\boldsymbol{z}} \in \mathrm{T}_{\boldsymbol{\varphi}_{\zeta}} \mathrm{M}$, such that $\omega(\boldsymbol{z}, \tilde{\boldsymbol{z}}) \neq 0$

Proof of Proposition 5.1. By the explicit form of $\omega(\cdot, \cdot)$ we have with $\zeta=(y, v, \vartheta, \mu)$

$$
\Omega_{\boldsymbol{\varphi}_{\zeta}}=\Omega_{\boldsymbol{\varphi}_{v, \mu}}, \text { and }\left(\Omega_{\boldsymbol{\varphi}_{v, \mu}}\right)_{j k}=-\left(\Omega_{\boldsymbol{\varphi}_{v, \mu}}\right)_{k j} \text {. }
$$

Thus, it suffices to consider elements in the tangent space with $y=0, \vartheta=0$. By the anti-symmetry of $\Omega_{\boldsymbol{\varphi}_{v, \mu}}$, it is sufficient to calculate the upper half triangle of the matrix. Without loss of generality we may choose coordinates so that $v$ is parallel to $x_{3}$-axis, where $x=\left(x_{1}, x_{2}, x_{3}\right)$. That is, $v=|v| \hat{e}_{3}$ and for such $v$ we use the notation $\varphi_{v, \mu}^{(3)}$ and $\Omega^{(3)}$.

The determinant of $\Omega_{\varphi_{v, \mu}}$ will be expressed in terms of $\tau, n_{, v}$ and $n_{, \mu}$, where

$$
\begin{gathered}
\tau_{j k}:=\omega\left(\partial_{x_{j}} \boldsymbol{\varphi}_{v, \mu}, \partial_{v_{k}} \boldsymbol{\varphi}_{v, \mu}\right), \quad\left(n_{, v}\right)_{j}:=-\omega\left(\partial_{x_{j}} \boldsymbol{\varphi}_{v, \mu}, \partial_{\mu} \boldsymbol{\varphi}_{v, \mu}\right), j, k=1,2,3, \\
n_{, \mu}\left(\boldsymbol{\varphi}_{v, \mu}^{(3)}\right):=\frac{1}{2} \partial_{\mu}\left\|\boldsymbol{\varphi}_{v, \mu}\right\|_{2}^{2} .
\end{gathered}
$$

Here $n(\mu, v):=\mathcal{N}\left(\boldsymbol{\varphi}_{v, \mu}\right)$. The relations (4.5) yield the identities

$$
\tau_{j k}=\left(L_{v, \mu} \partial_{v_{j}} \boldsymbol{\varphi}_{v, \mu}, \partial_{v_{k}} \boldsymbol{\varphi}_{v, \mu}\right)_{2}, \quad\left(n_{, v}\right)_{j}=\left(\partial_{v_{j}} \boldsymbol{\varphi}_{v, \mu}, \boldsymbol{\varphi}_{v, \mu}\right)_{2}, j, k=1,2,3 .
$$

The last equation yields $\left(n_{, v}\right)_{j}=n_{, v_{j}}$. Once the coordinates $v=|v| \hat{e}_{3}$ are chosen, we obtain $\tau^{(3)}, n_{, v}^{(3)}$ and $n_{, \mu}^{(3)}$, the corresponding notation for $\boldsymbol{\varphi}_{v, \mu}$ is $\boldsymbol{\varphi}_{v, \mu}^{(3)}$. 
Each element in $\Omega_{\boldsymbol{\varphi}_{\zeta}}$ is an integral of a product between a pair of tangent vectors. The reflection symmetry of the tangent vectors, shown under Assumption 2.1 in Proposition 4.3 , is the key to this proposition. We have

$$
\begin{aligned}
& \partial_{x_{1}} \boldsymbol{\varphi}_{v, \mu}^{(3)} \sim(\text { oee }, \text { oеo }), \partial_{x_{2}} \boldsymbol{\varphi}_{v, \mu}^{(3)} \sim(\text { eоe }, \text { eоo }), \partial_{x_{3}} \boldsymbol{\varphi}_{v, \mu}^{(3)} \sim(\text { eеo, eеe }) \sim J \boldsymbol{\varphi}_{v, \mu}^{(3)}, \\
& \partial_{v_{1}} \boldsymbol{\varphi}_{v, \mu}^{(3)} \sim(\text { oeo }, \text { oеe }), \quad \partial_{v_{2}} \boldsymbol{\varphi}_{v, \mu}^{(3)} \sim(\text { eоo, eоe }), \quad \partial_{v_{3}} \boldsymbol{\varphi}_{v, \mu}^{(3)} \sim(\text { eеe, eеo }) \sim \partial_{\mu} \boldsymbol{\varphi}_{v, \mu}^{(3)} \text {. }
\end{aligned}
$$

Let us calculate the cross term $\omega\left(\partial_{x_{1}} \boldsymbol{\varphi}_{v, \mu}^{(3)}, \partial_{x_{2}} \boldsymbol{\varphi}_{v, \mu}^{(3)}\right)$. It is an integral over a product of functions with symmetries $J \partial_{x_{1}} \boldsymbol{\varphi}_{v, \mu}^{(3)} \sim($ oeo, oee $)$ and $($ eoe, eoo $) \sim \partial_{x_{2}} \boldsymbol{\varphi}_{v, \mu}^{(3)}$. Thus both components of $J \partial_{x_{1}} \boldsymbol{\varphi}_{v, \mu}^{(3)}$ are odd in the first variable whereas $\partial_{x_{2}} \boldsymbol{\varphi}_{v, \mu}^{(3)}$ is even, hence the integral over this product vanish. Analogously, most of the other integrals vanish and by repeated use of (5.7) and (5.4)-(5.6) we find the matrix

$$
\Omega^{(3)}=\left(\begin{array}{cccccccc}
0 & 0 & 0 & \tau_{11}^{(3)} & 0 & 0 & 0 & 0 \\
0 & 0 & 0 & 0 & \tau_{22}^{(3)} & 0 & 0 & 0 \\
0 & 0 & 0 & 0 & 0 & \tau_{33}^{(3)} & 0 & -n_{, v_{3}}^{(3)} \\
-\tau_{11}^{(3)} & 0 & 0 & 0 & 0 & 0 & 0 & 0 \\
0 & -\tau_{22}^{(3)} & 0 & 0 & 0 & 0 & 0 & 0 \\
0 & 0 & -\tau_{33}^{(3)} & 0 & 0 & 0 & n_{, v_{3}}^{(3)} & 0 \\
0 & 0 & 0 & 0 & 0 & -n_{, v_{3}}^{(3)} & 0 & -n_{, \mu}^{(3)} \\
0 & 0 & n_{, v_{3}}^{(3)} & 0 & 0 & 0 & n_{, \mu}^{(3)} & 0
\end{array}\right) .
$$

Its determinant is $\tilde{\kappa}(v, \mu):=\operatorname{det} \Omega^{(3)}=\left(\tau_{11}^{(3)} \tau_{22}^{(3)}\right)^{2}\left(\tau_{33}^{(3)} n_{, \mu}^{(3)}+\left(n_{, v_{3}}^{(3)}\right)^{2}\right)^{2}$. By Lemma 5.4 below there is an $0<\tilde{r}_{1} \leq r_{0} \leq 1$ such that $\tau_{j j}^{(3)}>0$, and by Part ((iv)) of Proposition 4.3 $n_{, \mu}^{(3)}>0$ for all $|v| \leq r_{0}$ and $\mu \in I_{0}$. Thus $\tilde{\kappa}>0$. Now, let $r_{1}<\tilde{r}_{1}$ and $I_{1}$ be an open non-empty subinterval of $I_{0}$ such that $\bar{I}_{1} \subset I_{0}$, then on the closed set $\left[0, r_{1}\right] \times \bar{I}_{1}, \tilde{\kappa}$ attains its minimum $\kappa>0$.

Corollary 5.3. The matrices $\Omega_{\boldsymbol{\varphi}_{v, \mu}}$ and $\Omega_{\boldsymbol{\varphi}_{v, \mu}^{-1}}^{-1}$ have the form

$$
\Omega_{\boldsymbol{\varphi}_{v, \mu}}=\left(\begin{array}{cccc}
0 & \tau & 0 & -n_{, v} \\
-\tau & 0 & n_{, v} & 0 \\
0 & -n_{, v}^{T} & 0 & -n_{, \mu} \\
n_{, v}^{T} & 0 & n_{, \mu} & 0
\end{array}\right), \Omega_{\boldsymbol{\varphi}_{v, \mu}}^{-1}=\left(\begin{array}{cccc}
0 & -g & 0 & q \\
g & 0 & -q & 0 \\
0 & q^{T} & 0 & -\gamma \\
-q^{T} & 0 & \gamma & 0
\end{array}\right),
$$

with $\tau, n_{, v}$ and $n_{, \mu}$ as in (5.4)-(5.5) and where

$$
g=\left(\tau+n_{, \mu}^{-1} n_{, v} n_{, v}^{T}\right)^{-1}, \quad q=\left(\tau n_{, \mu}+n_{, v} n_{, v}^{T}\right)^{-1} n_{, v}
$$

and

$$
\gamma=n_{, \mu}^{-1}\left(-1+n_{, v}^{T}\left(\tau n_{, \mu}+n_{, v} n_{, v}^{T}\right)^{-1} n_{, v}\right)
$$

Proof. To obtain $\Omega_{\varphi_{v, \mu}}$, we observe that each block matrix e.g., $\tau, n_{, v} n_{, \mu}$, is related to the corresponding matrix block in Eq. (5.8) by a change of coordinates. Thus, matrix blocks that in (5.8) are identically zero remain so, and $\tau, n_{, v}$ and $n_{, \mu}$ remain as in the general form from (5.4)-(5.5). 
Lemma 5.4. Let $\tau_{j j}^{(3)}$ be as in (5.4). There is a number $0<\tilde{r}_{1} \leq r_{0}$ such that $\tau_{j j}^{(3)}>0$ for $j=1,2,3$.

Proof. At $v=0, \tau^{(3)}$ reduces to

$$
\left.\tau_{j j}\right|_{v=0}=\left(\beta_{j}, L_{22, \mu} \beta_{j}\right)_{2}
$$

where $\beta_{j}$ is defined through $\boldsymbol{\beta}_{j}:=\left.\partial_{v_{j}} \boldsymbol{\varphi}_{v, \mu}\right|_{v=0}$ and $\boldsymbol{\beta}_{j}=\left(0, \beta_{j}\right)$. The linear operator $L_{22, \mu} \geq 0$ has a non-degenerate zero eigenvalue, with corresponding eigenfunction is $\varphi_{\mu}$, see the proof of Proposition A.1. But, since $\beta_{j} \sim \partial_{x_{j}} \varphi_{\mu}$ we have $\beta_{j} \perp \varphi_{\mu}$ and hence $\left.\tau_{j j}\right|_{v=0}>0$. By the continuity of $\tau_{j j}^{(3)}$ in $v$, there is a number $\tilde{r}_{1 j}>0$ such that for all $|v|<\tilde{r}_{1 j}$ we have that $\tau_{j j}^{(3)}>0$. Now let $\tilde{r}_{1}=\min _{j} \tilde{r}_{1 j}$.

\section{Symplectically orthogonal decomposition}

In this section we introduce the symplectically (or skew) orthogonal decomposition of a function $\boldsymbol{\psi}$ close to the soliton manifold. The decomposition has two components, one on the manifold, $\boldsymbol{\varphi}_{\varsigma(\boldsymbol{\psi})}$, and one in the symplectically orthogonal direction, $\boldsymbol{\xi}$. We show that the decomposition uniquely defines the modulation parameter $\zeta=(y, v, \vartheta, \mu)$ and a perturbation $\boldsymbol{\xi}$.

Recall from Proposition 5.1 that the modulation parameter $\zeta$ is a point in the parameter space $\mathrm{Z}_{1}=\mathrm{Z}\left(r_{1}, I_{1}\right)$ and

$$
\mathrm{Z}\left(r_{1}, I_{1}\right):=\mathbb{R}^{3} \times B_{r_{1}}^{3}(0) \times[0,2 \pi) \times I_{1} .
$$

All ground states described by the modulation parameters in $\mathrm{Z}_{1}$ define the soliton manifold $\mathrm{M}_{1}:=\mathrm{M}\left(\mathrm{Z}_{1}\right)$. Above, $B_{r}^{n}(0) \subset \mathbb{R}^{n}$ denotes an open ball of radius $r$ and $I_{1}$ is an open interval on $\mathbb{R}$. The element $\vartheta$ in $\zeta$ is a phase, and we can replace its domain $[0,2 \pi)$ with $\mathrm{S}^{1}$. With this replacement we note that only the velocity, $v$, and the frequency, $\mu$, parameter have the constrained domains $B_{r}^{3}$ and $I_{1}$ respectively. The dependence of the solitary waves on the parameters $v$ and $\mu$ requires our attention (see Corollary 6.5 below) when constructing a 'uniform' tubular neighborhood, $U_{\delta}$, of the soliton manifold, where the decomposition exists and is unique. For subsets $\mathrm{Z}_{3} \subset \mathrm{Z}_{2} \subset \mathrm{Z}_{1}$, to be introduced below, we define the tubular neighborhood $U_{\delta}=U_{\delta}\left(\mathrm{Z}_{j}\right)$ of $\mathrm{M}\left(\mathrm{Z}_{j}\right)$ by

$$
U_{\delta}\left(\mathrm{Z}_{j}\right):=\left\{\boldsymbol{\psi} \in \tilde{\Gamma}: \inf _{\zeta \in \mathrm{Z}_{j}}\left\|\boldsymbol{\psi}-\boldsymbol{\varphi}_{\zeta}\right\|_{\tilde{\Gamma}}<\delta\right\}, j=2,3
$$

Let $b_{R}$ be an open ball around $\boldsymbol{\varphi}_{\zeta}$ in the phase space $\tilde{\Gamma}$ with radius $R$, defined by

$$
b_{R}\left(\boldsymbol{\varphi}_{\zeta}\right):=\left\{\boldsymbol{\psi} \in \tilde{\Gamma}:\left\|\boldsymbol{\psi}-\boldsymbol{\varphi}_{\zeta}\right\|_{\tilde{\Gamma}}<R\right\}
$$

where $\tilde{\Gamma}:=\left\{\boldsymbol{\psi} \in \mathrm{H}^{\frac{1}{2}}:\|\boldsymbol{\psi}\|_{\tilde{\Gamma}}<\infty\right\}$, see also (2.11).

We have the result: 
Proposition 6.1. Suppose Assumption 2.1 is satisfied and let $\mathrm{Z}_{1}$ be defined as above. Given $\delta>0$ sufficiently small, and let $r_{2}$ and $r_{3}$ be such that $0<r_{3}<r_{2}<r_{1}$ and let $I_{2}$, $I_{3}$ be open non-empty intervals with $\bar{I}_{j+1} \subset I_{j}, j=1,2$, with corresponding parameter domains $\mathrm{Z}_{2}, \mathrm{Z}_{3}$ and soliton manifolds $\mathrm{M}_{2}, \mathrm{M}_{3}$. Then for every $\boldsymbol{\psi} \in U_{\delta}\left(\mathrm{Z}_{k+1}\right)$ and $k=1,2$ there is a unique $\mathrm{C}^{1}\left(U_{\delta}\left(\mathrm{Z}_{k+1}\right), \mathrm{Z}_{k}\right)$-map $\varsigma$ such that

(i) For each $\boldsymbol{\psi} \in U_{\delta}\left(\mathrm{Z}_{k+1}\right)$, we have

$$
\omega\left(\boldsymbol{\psi}-\boldsymbol{\varphi}_{\varsigma(\boldsymbol{\psi})}, \boldsymbol{z}\right)=0, \forall \boldsymbol{z} \in \mathrm{T}_{\boldsymbol{\varphi}_{\varsigma(\psi)}} \mathrm{M}_{k}
$$

Furthermore, for each $\boldsymbol{\psi} \in U_{\delta}\left(\mathrm{Z}_{k+1}\right)$ there exists a $\zeta^{(0)} \in \mathrm{Z}_{k}$ such that $\boldsymbol{\varphi}_{\zeta^{(0)}}$ is the orthogonal projection of $\boldsymbol{\psi}$ onto $\mathrm{M}_{k}$, and

(ii) $\left\|\boldsymbol{\varphi}_{\varsigma(\boldsymbol{\psi})}-\boldsymbol{\varphi}_{\zeta^{(0)}}\right\|_{\tilde{\Gamma}} \leq C \delta$,

(iii) $\left|\zeta^{(0)}-\varsigma(\boldsymbol{\psi})\right| \leq C \delta$

for some positive constant $C>0$.

The above proposition defines a unique function $\varsigma: U_{\mathrm{Z}_{k+1}} \rightarrow \mathrm{Z}_{k}$. Consequently, $\boldsymbol{\psi} \mapsto(\varsigma, \boldsymbol{\xi})$ with $\boldsymbol{\xi}:=\boldsymbol{\psi}-\boldsymbol{\varphi}_{\varsigma(\boldsymbol{\psi})}$ defines a unique decomposition of $\boldsymbol{\psi} \in U_{\delta}\left(\mathrm{Z}_{2}\right)$.

Remark 6.2. Given $r_{j}$ and $I_{j}$ for $j=1,2,3$, we have above determined a $\delta>0$ such that $\varsigma(\boldsymbol{\psi}), \zeta^{(0)}(\boldsymbol{\psi}) \in \mathrm{Z}_{j-1}$, when $\boldsymbol{\psi} \in U_{\delta}\left(\mathrm{Z}_{j}\right)$. Thus we can now give the relation between the distances $\delta_{\mathrm{M}}\left(\delta_{P}\right)$, the minimal distance between the manifolds and $\delta$ introduced above (See Section 11, Figure 3.1). This relation is $\delta_{\mathrm{M}} \leq c \delta, \delta_{P} \leq c \delta$. For some constant $c>0$, which partly is determined by the size of $C$ in part (ii) and (iii) above.

Remark 6.3. If we choose in Proposition 6.1 an even smaller distance, $\varepsilon<\delta$, and consider the tubular neighborhood $U_{\varepsilon}\left(\mathrm{Z}_{3}\right)$, then part $(i i)$ and $($ iii $)$ above hold with $\delta$ replaced by $\varepsilon$.

The proof of Proposition 6.1 needs four intermediate results and is given in the end of this section. We define the function $G: \mathrm{H}^{\frac{1}{2}} \times \mathrm{Z}_{1} \rightarrow \mathbb{R}^{8}$ by

$$
G_{j}(\boldsymbol{\psi}, \zeta):=\omega\left(\boldsymbol{\psi}-\boldsymbol{\varphi}_{\zeta}, \boldsymbol{z}_{j, \zeta}\right), \quad j=1, \ldots, 8,
$$

where $\boldsymbol{z}_{j, \zeta}$ is the $j$ :th tangent vector in $\mathrm{T}_{\boldsymbol{\varphi}_{\zeta}} \mathrm{M}_{1}$ to the soliton manifold at the point $\boldsymbol{\varphi}_{\zeta}$, see the ordering given by the list (5.1). We will consider solutions to the equation $G=0$ that are close to a solution $(\boldsymbol{\psi}, \zeta) \mapsto\left(\boldsymbol{\varphi}_{\zeta^{(c)}}, \zeta^{(c)}\right)$. We introduce the notation $\zeta^{(c)}$ to distinguish an arbitrary parameter $\zeta$ from the center position, $\zeta^{(c)}$, of the ball where we solve the equation $G=0$. We have the first result:

Lemma 6.4. Suppose Assumption 2.1 is satisfied. Then, for every center position $\zeta^{(c)} \in$ $\mathrm{Z}_{1}$ there are balls $b_{R_{1}}\left(\boldsymbol{\varphi}_{\zeta^{(c)}}\right)$ and $B_{\rho_{1}}^{8}\left(\zeta^{(c)}\right)$ in $\tilde{\Gamma}$ and $\mathrm{Z}_{1}$, respectively, with centers $\boldsymbol{\varphi}_{\zeta^{(c)}}$, $\zeta^{(c)}$ and radii $R_{1}, \rho_{1}$ and a unique $\mathrm{C}^{1}\left(b_{R_{1}}, B_{\rho_{1}}^{8}\right)$ map, such that $G(\boldsymbol{\psi}, \varsigma(\boldsymbol{\psi}))=0$ for all $\boldsymbol{\psi} \in b_{R_{1}}\left(\boldsymbol{\varphi}_{\zeta^{(c)}}\right)$. Both $R_{1}$ and $\rho_{1}$ depend on the center position $\zeta^{(c)}$. 
Proof. We use an implicit function theorem to solve the equation $G=0$. We need to show that $(a) G$ is $\mathrm{C}^{1},(b) G\left(\boldsymbol{\varphi}_{\zeta^{(c)}}, \zeta^{(c)}\right)=0$ and $\left.(c) \partial_{\zeta} G\left(\boldsymbol{\varphi}_{\zeta^{(c)}}, \zeta\right)\right|_{\zeta=\zeta^{(c)}}$ is invertible.

(a) $G$ is $\mathrm{C}^{1}$ in $\boldsymbol{\psi}$ since it is linear in $\boldsymbol{\psi} . G$ is $\mathrm{C}^{1}$ in $\zeta$ since both $\boldsymbol{\varphi}_{\zeta}$ and $\boldsymbol{z}_{j, \zeta}$ are $\mathrm{C}^{1}$ in $\zeta$ see Proposition 4.3;

(b) follows from the definition of $G$;

(c) calculate

$$
\partial_{\zeta_{k}} G_{j}(\boldsymbol{\psi}, \zeta)=-\omega\left(\partial_{\zeta_{k}} \boldsymbol{\varphi}_{\zeta}, \boldsymbol{z}_{j, \zeta}\right)+\omega\left(\boldsymbol{\psi}-\boldsymbol{\varphi}_{\zeta}, \partial_{\zeta_{k}} \boldsymbol{z}_{j, \zeta}\right)
$$

at $(\boldsymbol{\psi}, \zeta)=\left(\boldsymbol{\varphi}_{\zeta^{(c)}}, \zeta^{(c)}\right)$

$$
\left.\partial_{\zeta_{k}} G_{j}\left(\boldsymbol{\psi}, \zeta^{(c)}\right)\right|_{\psi=\boldsymbol{\varphi}_{\zeta^{(c)}}}=-\omega\left(\partial_{\zeta_{k}} \boldsymbol{\varphi}_{\zeta}, z_{\zeta, j}\right)
$$

Choose coordinate axis such that $v$ is parallel to $x_{3}$ then

$$
\left.\partial_{\zeta_{k}} G_{j}\left(\boldsymbol{\psi}, \zeta^{(c)}\right)\right|_{\psi=\boldsymbol{\varphi}_{\zeta}(c)}=-\Omega_{\boldsymbol{\varphi}_{\zeta(c)}},
$$

with $\Omega_{\boldsymbol{\varphi}_{\zeta(c)}}$ as in Section 5. Thus, by Proposition 5.1, $\operatorname{det} \Omega_{\boldsymbol{\varphi}_{\zeta^{(c)}}}>\kappa>0$ and we have shown $(c)$.

All assumptions in the implicit function theorem are thus satisfied and, therefore there are open neighborhoods $W \subset \tilde{\Gamma}$ and $V \subset \mathrm{Z}_{1}$ around $\boldsymbol{\varphi}_{\zeta}$ and $\zeta$ respectively and an unique $\mathrm{C}^{1}$-function, $\varsigma: W \rightarrow V$ such that for all $\boldsymbol{\psi} \in W G(\boldsymbol{\psi}, \varsigma(\boldsymbol{\psi}))=0$. Now choose $R_{1}$ sufficiently small so that $b_{R_{1}} \subset W$ and $\rho_{1}$ sufficiently large so that $V \subset B_{\rho_{1}}^{8}$. By possibly reducing $R_{1}$ further (and consequently $\rho_{1}$ by continuity), we find that $B_{\rho_{1}}^{8} \subset \mathrm{Z}_{1}$.

To single out a tubular neighborhood $U_{\delta}$ around the soliton manifold of constant 'width' $\delta$, we examine in the next two corollaries how the radii in Lemma 6.4 depend on the parameters. First, by using symmetries of $G$ we have:

Corollary 6.5. For every $\zeta^{(c)} \in \mathrm{Z}_{1}$, the radii of Lemma 6.4 only depend of $\mu$ and $v$.

Proof. The function $G$ is invariant under translation in the sense that if a parameter $y$ of $\zeta$ maps to $y+a$ and $\boldsymbol{\psi}(x) \mapsto \boldsymbol{\psi}(x-a)$ then the value of $G$ is unchanged. This implies that the balls $b_{R_{1}}$ and $B_{\rho_{1}}^{8}$ are independent of which position $y$ they are calculated for.

Analogously, $G$ is phase invariant in the sense that $\vartheta \mapsto \vartheta+\gamma$ and $\boldsymbol{\psi} \mapsto \mathrm{e}^{-\gamma J} \boldsymbol{\psi}$ leave $G$ unchanged. Thus $R_{1}=R_{1}(v, \mu)$ and $\rho_{1}=\rho_{1}(v, \mu)$.

To achieve uniform radii in Lemma 6.4, we have the following result:

Corollary 6.6. There are a number $r_{2}^{\prime}, 0<r_{2}^{\prime}<r_{1}$, and a non-empty open interval $I_{2}^{\prime} \Subset I_{1}$ such that if the $\zeta \in \mathrm{Z}\left(r_{2}^{\prime}, I_{2}^{\prime}\right)$, then the result of Lemma 6.4 holds with uniform radii. Furthermore, for sufficiently small $\rho_{2}$ there exists $c>0$ such that

$$
R_{2} \leq c \rho_{2} .
$$

Uniform here implies that $R_{2}, \rho_{2}$ (and c) only depend on $r_{1}, r_{2}^{\prime}, I_{1}$ and $I_{2}^{\prime}$. 
Let $\mathrm{Z}_{2}^{\prime}:=\mathrm{Z}\left(r_{2}^{\prime}, I_{2}^{\prime}\right)$. The proof of this corollary is somewhat tedious and is placed in Appendix C. It relies on two observations. First, the ground state and maps thereof are well defined and have a $\mathrm{C}^{1}$ dependence on the parameters on the whole manifold $\mathrm{M}_{1}$. This allows us to extract uniform radii away from the boundaries. Second, by choice of $r_{2}^{\prime}$ and $I_{2}$, the only constrained directions, we are a fixed distance away from the boundaries of $\mathrm{M}_{1}$ and $\mathrm{Z}_{1}$, respectively. Thus we can find a uniform radii on this smaller set.

The next lemma is captured by Figure 6.1.

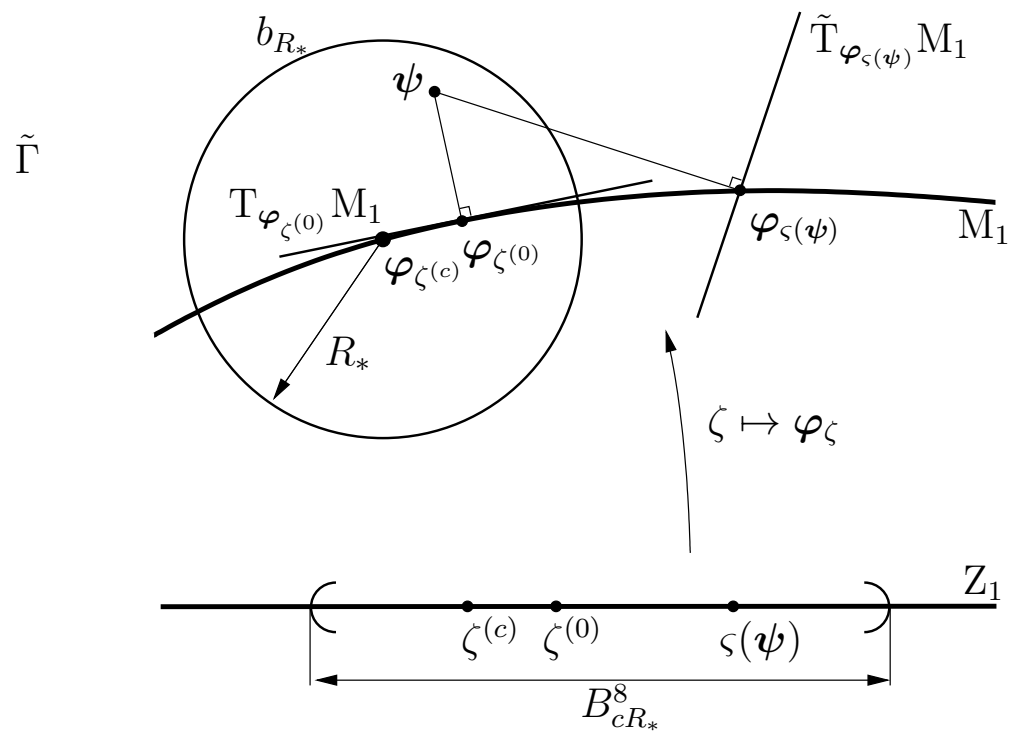

Figure 6.1: The above figure displays the sets $\mathrm{Z}_{1}$ and $\mathrm{M}_{1}$, as well as $\mathrm{T}_{\boldsymbol{\varphi}_{\zeta(0)}} \mathrm{M}_{1}$ and the symplectically orthogonal plane $\tilde{\mathrm{T}}_{\boldsymbol{\varphi}_{\varsigma(\psi)}} \mathrm{M}_{1}$. Furthermore, we schematically sketch the orthogonal and symplectically orthogonal decomposition of $\boldsymbol{\psi}$.

Lemma 6.7. Suppose that Assumption 2.1 is satisfied. There are numbers $0<R_{*}<R_{2}$ and $0<r_{2}<r_{2}^{\prime}$ as well as a non-empty open interval $I_{2} \Subset I_{2}^{\prime}$ such that the following holds. If $\boldsymbol{\psi} \in b_{R_{*}}\left(\boldsymbol{\varphi}_{\zeta^{(c)}}\right), \zeta^{(c)} \in \mathrm{Z}\left(r_{2}, I_{2}\right)$, then there exists a unique $\zeta^{(0)} \in \mathrm{Z}_{1}$ such that

1. $\boldsymbol{\varphi}_{\zeta^{(0)}}$ minimizes $\left\|\boldsymbol{\psi}-\boldsymbol{\varphi}_{\zeta}\right\|_{\tilde{\Gamma}}$;

2. $\left\|\varphi_{\varsigma(\psi)}-\varphi_{\zeta^{(0)}}\right\|_{\tilde{\Gamma}} \leq C R_{*}$;

3. $\left|\varsigma(\boldsymbol{\psi})-\zeta^{(0)}\right| \leq C R_{*}$.

Here $C$ depends only on $r_{1}, r_{2}, r_{2}^{\prime}, I_{1}$ and $I_{2}, I_{2}^{\prime}$.

Proof. First note that $\tilde{\Gamma}$ is a Hilbert space. There exists $0<r_{1}^{\prime \prime}<r_{0}$ and a non-empty open interval $I_{1}^{\prime \prime} \Subset I_{0}$, with corresponding domain $\mathrm{Z}_{1}^{\prime \prime}:=\mathrm{Z}\left(r_{1}^{\prime \prime}, I_{1}^{\prime \prime}\right)$ and $\mathrm{M}_{1}^{\prime \prime}:=\mathrm{M}\left(\mathrm{Z}_{1}^{\prime \prime}\right)$ such that for $\boldsymbol{\psi}$ that in $\|\cdot\|_{\tilde{\Gamma}}$-norm are sufficiently close to $\mathrm{M}_{1}^{\prime \prime}$ the orthogonal projection of $\boldsymbol{\psi}$ onto $\mathrm{M}_{0}$ exists and is unique. To see this, define $f_{\boldsymbol{\psi}}(\zeta):=\left\|\boldsymbol{\psi}-\boldsymbol{\varphi}_{\zeta}\right\|_{\tilde{\Gamma}}^{2}$. Observe that $f_{\psi} \in \mathrm{C}^{2}\left(\mathrm{Z}_{0}, \mathbb{R}\right)$, and that $\mathrm{Z}_{0}$ is a convex open set. To see that $f_{\psi}$ has a minimum, 
it suffices to show that (i) there exists a $\zeta^{(0)}=\zeta^{(0)}(\boldsymbol{\psi})$ such that $f_{\boldsymbol{\psi}}^{\prime}\left(\zeta^{(0)}\right)=0$ and (ii) $f_{\boldsymbol{\psi}}^{\prime \prime}(\zeta)>0$, for all $\zeta \in \mathrm{Z}_{0}$. To show (ii), we calculate

$$
\left(f_{\boldsymbol{\psi}}^{\prime \prime}(\zeta)\right)_{j k}=\left(\boldsymbol{\psi}-\boldsymbol{\varphi}_{\zeta}, \partial_{\zeta_{j}} \boldsymbol{z}_{k, \zeta}\right)_{\tilde{\Gamma}}+\left(\boldsymbol{z}_{j, \zeta}, \boldsymbol{z}_{k, \zeta}\right)_{\tilde{\Gamma}}
$$

where $\boldsymbol{z}_{k, \zeta}:=\partial_{\zeta_{k}} \boldsymbol{\varphi}_{\zeta}$. The last term, $\left(f_{\boldsymbol{\varphi}_{\zeta}}^{\prime \prime}(\zeta)\right)_{j k}=\left(\boldsymbol{z}_{j, \zeta}, \boldsymbol{z}_{k, \zeta}\right)_{\tilde{\Gamma}} \operatorname{simplifies~to~}\left(\boldsymbol{z}_{j, v, \mu}, \boldsymbol{z}_{k, v, \mu}\right)_{\tilde{\Gamma}}$, since phase and translation vanish in the integral. By the reflection symmetries of $\boldsymbol{z}_{k, v, \mu}$, see Proposition 4.3(ii), together with the fact that both $|x|$ and $\sqrt{1-\Delta}$ commutes with rotation and reflection, we find that $\operatorname{det} f_{\boldsymbol{\varphi}_{v, \mu}}^{\prime \prime}(\zeta)>0$ for $|v| \leq r_{1}^{\prime \prime}$, for some $0<r_{1}^{\prime \prime}<r_{0}$ and $\mu \in I_{1}^{\prime \prime}$, and $I_{1}^{\prime \prime}$ open an non-empty, such that $I_{1}^{\prime \prime} \Subset I_{0}$. To extend the positivity of $f_{\boldsymbol{\varphi}_{v, \mu}}^{\prime \prime}$ to positivity of $f_{\boldsymbol{\psi}}^{\prime \prime}$, we see that the first term in (6.10) can be made small, when $\left\|\boldsymbol{\psi}-\boldsymbol{\varphi}_{\zeta}\right\|_{\tilde{\Gamma}}$ is small. Thus, for $\boldsymbol{\psi}$ sufficiently close to $\mathrm{M}\left(\mathrm{Z}\left(r_{1}^{\prime \prime}, I_{1}^{\prime \prime}\right)\right)$ we have $f_{\boldsymbol{\psi}}^{\prime \prime}>0$ and we have proved (ii). To show (i), we note that $f_{\boldsymbol{\varphi}_{\zeta}}^{\prime}(\zeta)=0, f_{\boldsymbol{\varphi}_{\zeta}}^{\prime \prime}>0$ and $f_{\boldsymbol{\psi}}^{\prime} \in \mathrm{C}^{1}$ thus we can use the implicit function theorem to find a small neighborhood of $\boldsymbol{\varphi}_{\zeta}$ where there is a $\mathrm{C}^{1}$-map $\zeta^{(0)}(\boldsymbol{\psi})$ such that $f_{\boldsymbol{\psi}}\left(\zeta^{(0)}\right)=0$. Furthermore, this map $\zeta^{(0)}$ is unique and we have shown (i). Thus close to the manifold there exists a unique minimizer.

Now repeating the minimization procedure and replace $\mathrm{Z}_{0}$ with the smaller domain $\mathrm{Z}_{2}^{\prime}$ we find that there is a orthogonal minimizer on $\mathrm{Z}_{2}^{\prime \prime}$, where $\mathrm{Z}_{2}^{\prime \prime}:=\mathrm{Z}\left(r_{2}^{\prime \prime}, I_{2}^{\prime \prime}\right)$ for some $0<r_{2}^{\prime \prime}<r_{2}^{\prime}$ and $I_{2}^{\prime \prime}$ non-empty and open, $I_{2}^{\prime \prime} \Subset I_{2}^{\prime}$. Let $\mathrm{M}_{2}^{\prime \prime}:=\mathrm{M}\left(\mathrm{Z}_{2}^{\prime \prime}\right)$.

The projection on $\mathrm{M}_{2}^{\prime \prime}$ is denoted by $\boldsymbol{\varphi}_{\zeta^{(0)}}$ and the inverse of the map $\zeta \mapsto \boldsymbol{\varphi}_{\zeta}$ is the coordinate map of the manifold and uniquely defines $\zeta^{(0)}$. The corresponding radii $R_{3}$, $\rho_{3}$ of balls in $\tilde{\Gamma}$ and $\mathbb{R}^{8}$ respectively depends on the centrum point $\boldsymbol{\varphi}_{\zeta^{(c)}}$ respectively $\zeta^{(c)}$ around which the implicit function theorem above is constructed. Analogously to the proof of Corollary 6.6 we can reduce the domain of center positions, $\zeta^{(c)} \in \mathrm{Z}\left(r_{2}, I_{2}\right)$, for some $0<r_{2}<r_{2}^{\prime \prime}, I_{2} \Subset I_{2}^{\prime \prime}$ and thus find uniform radii $R_{4}, \rho_{4}$ where the decomposition is valid. Moreover, by continuity of the the map $\zeta^{(0)}(\psi)$ we have $R_{4}=c \rho_{4}$ for some $c>0$. Let $\mathrm{Z}_{2}:=\mathrm{Z}\left(r_{2}, I_{2}\right)$.

We now show part 2 and 3 . Let $R_{*}=\min \left(R_{4}, R_{2}\right)$ (where $R_{2}$ is the radius where the symplectic decomposition is valid). For $\boldsymbol{\psi} \in b_{R_{*}}\left(\boldsymbol{\varphi}_{\zeta^{(c)}}\right), \zeta^{(c)} \in \mathrm{Z}_{2}$ both the orthogonal and the symplectic projection is well defined. We note that $\boldsymbol{\varphi}_{\zeta^{(0)}} \in b_{R_{*}}\left(\boldsymbol{\varphi}_{\zeta^{(c)}}\right)$ and that $\left\|\boldsymbol{\varphi}_{\varsigma(\psi)}-\boldsymbol{\varphi}_{\zeta^{(c)}}\right\|_{\tilde{\Gamma}} \leq C^{\prime} R_{*}$ thus

$$
\left\|\boldsymbol{\varphi}_{\zeta^{(0)}}-\boldsymbol{\varphi}_{\varsigma(\psi)}\right\|_{\tilde{\Gamma}} \leq C R_{*}
$$

Furthermore, since $\zeta^{(0)} \in B_{c^{\prime} R_{*}}^{8}\left(\zeta^{(c)}\right)$ and $\varsigma(\boldsymbol{\psi}) \in B_{c^{\prime \prime} R_{*}}^{8}\left(\zeta^{(c)}\right)$, it follows that $\left|\zeta^{(0)}-\varsigma(\boldsymbol{\psi})\right| \leq$ $C R_{*}$.

Proof of Proposition 6.1. We first note that $r_{2}<r_{2}^{\prime}, I_{2} \Subset I_{2}^{\prime}$, with corresponding $\mathrm{Z}_{2}=$ $\mathrm{Z}\left(r_{2}, I_{2}\right)$. With $R_{*}>0$ as chosen in Lemma 6.7 we note that $U_{R_{*}}(\mathrm{Z})$, is the union of balls $b_{R_{*}}\left(\boldsymbol{\varphi}_{\zeta^{(c)}}\right)$ over all central points in $\zeta^{(c)} \in \mathrm{Z}_{2}$. By Lemma 6.7, there exists a point $\zeta^{(0)} \in \mathrm{Z}_{2}$ such that $\boldsymbol{\varphi}_{\zeta^{(0)}}$ is the orthogonal projection of $\boldsymbol{\psi}$ onto $\mathrm{M}_{1}$. Part (2) and (3) of this lemma ensure that (ii) and (iii) of the Proposition 6.1 is satisfied with $\delta=R_{*}$.

Lemma 6.4 on the smaller balls, with radii $R_{*}$ and $\rho_{2}=c R_{*}$ chosen as above, shows that in each such ball there exists a unique $\mathrm{C}^{1}$ map $\varsigma$, with the property that

$$
\omega\left(\boldsymbol{\psi}-\boldsymbol{\varphi}_{\varsigma(\psi)}, \boldsymbol{z}\right)=0
$$


for all $\boldsymbol{z} \in \mathrm{T}_{\boldsymbol{\varphi}_{\varsigma(\psi)}} \mathrm{M}_{1}$. As shown above, $\varsigma$ is unique in each ball, hence it is unique in $U_{\delta}\left(\mathrm{Z}_{2}\right)$, with $\delta=R_{*}$.

That the decomposition exists for $\boldsymbol{\psi}$ in an even smaller set, $\mathrm{M}\left(\mathrm{Z}_{3}\right)$, with $\mathrm{Z}_{3}=\mathrm{Z}\left(r_{3}, I_{3}\right)$, $0<r_{3}<r_{2}, I_{3}$ non-empty, open interval, $I_{3} \Subset I_{2}$, is clear. To show that the resulting decomposition map $\varsigma \in \mathrm{Z}_{2}$ rather than in $\mathrm{Z}_{1}$ and verify Part (ii) and (iii) of this proposition for this smaller set, is done by repeating the above lemmas and corollaries with $\mathrm{Z}_{1}$ replaced with $\mathrm{Z}_{2}$. The resulting radius $\delta>0$, with $\boldsymbol{\psi} \in b_{\delta}\left(\boldsymbol{\varphi}_{\zeta^{(c)}}\right)$ will be smaller or equal to $R_{*}$ constructed above.

Due to the uniform radii constructed above, we find that the constant $C$ in the Part (ii) and (iii) of the proposition depend only on the $r$ 's and the I's constructed above and not on the points $\varsigma$ or $\zeta^{(0)}$. We have proved the proposition.

\section{Dynamics in a moving frame}

In this section we apply the unique decomposition provided by Proposition 6.1 to a class of solutions of (1.1) and find the resulting equations for the decomposed parts. Another way to see this decomposition is that we make the change of variables $\boldsymbol{\psi} \mapsto(\zeta, \boldsymbol{\xi})$ and derive the equation of motion for this set of variables. As mentioned above, $\boldsymbol{\xi}$ can be seen as a perturbation to a solitary wave parameterized by $\zeta$.

For the decomposition of a solution $\boldsymbol{\psi}$ to Eq. (1.1) to exist, we require that $\boldsymbol{\psi}$ to remain in the tubular neighborhood $U_{\delta}\left(\mathrm{Z}_{2}\right)$ for some interval of times. This is ensured by the requirement that the corresponding initial condition $\boldsymbol{\psi}_{0}$ belongs to $U_{\delta}\left(\mathrm{Z}_{3}\right)$. The

decomposition is defined by the unique function $\varsigma(\boldsymbol{\psi})$, with $\varsigma=(y, v, \vartheta, \mu)$ that solves the equation $G(\boldsymbol{\psi}, \zeta)=0$, where $G(\boldsymbol{\psi}, \zeta):=\omega\left(\boldsymbol{\psi}-\boldsymbol{\varphi}_{\zeta}, \boldsymbol{z}_{\cdot, \zeta}\right)$, and the relation

$$
\boldsymbol{\psi}(x, t)=\mathrm{e}^{-\vartheta(t) J}\left(\boldsymbol{\varphi}_{v(t), \mu(t)}(x-y(t))+\boldsymbol{\xi}(x-y(t), t)\right) .
$$

Thus, the existence of $\varsigma$ ensures that $\omega(\boldsymbol{\xi}, \boldsymbol{z})=0$ for all $\boldsymbol{z}$ in $\mathrm{T}_{\boldsymbol{\varphi}_{v, \mu}} \mathrm{M}_{2}$. Here

$$
\mathrm{e}^{-\vartheta J}=\left(\begin{array}{cc}
\cos \vartheta & -\sin \vartheta \\
\sin \vartheta & \cos \vartheta
\end{array}\right)
$$

The solution $\boldsymbol{\psi}$ depends on time, and consequently so does $\zeta(t):=\varsigma(\boldsymbol{\psi}(\cdot, t))$ and $\boldsymbol{\xi}$.

By substituting the decomposition (7.1) into the Eq. (2.15) (which is the Hamiltonian formulation of (1.1)) and upon applying 'projections' of the symplectic form we have the result:

Proposition 7.1. Let $U_{\delta}\left(\mathrm{Z}_{3}\right)$ be defined as above. Suppose that Assumption 2.1 is satisfied. Let $\boldsymbol{\psi}(\cdot, t)$ be a solution to $(2.15)$ with initial condition $\boldsymbol{\psi}_{0} \in U_{\delta}\left(\mathrm{Z}_{3}\right)$ and let $\boldsymbol{\xi}(\cdot, t)$ and $\zeta(t)=(y(t), v(t), \vartheta(t), \mu(t))$ be the decomposed parameters corresponding to $\boldsymbol{\psi}(\cdot, t)$. Furthermore, let the external potential $V$ satisfy (3.1) for some $\varepsilon>0$. Then,

(i) the parameters $\zeta=(y, v, \vartheta, \mu)$ satisfy the modulation equations

$$
\alpha_{j}+\mathcal{N}\left(\boldsymbol{\varphi}_{v, \mu}\right) \sum_{\nu=1}^{3}\left(\Omega_{\boldsymbol{\varphi}_{v, \mu}}\right)_{j \nu}^{-1} \partial_{y_{\nu}} V(y)=Y_{j}
$$


where

$$
\alpha:=(v-\dot{y}, \dot{v}, \mu-\dot{\vartheta}-V(y), \dot{\mu}),
$$

and the perturbation terms $Y_{j}$ are given as the right-hand side of (7.23) and satisfies the estimate

$$
|Y| \leq C\left(\|\boldsymbol{\xi}\|_{\mathrm{H}^{\frac{1}{2}}}^{3}+\|\boldsymbol{\xi}\|_{\mathrm{H}^{\frac{1}{2}}}^{2}+\varepsilon^{2}+|\alpha|\|\boldsymbol{\xi}\|_{2}\right) .
$$

Furthermore, we have $|\alpha| \leq C \varepsilon+|Y|$. Here $\Omega_{\varphi_{v, \mu}}$ is the matrix (5.9) and $n(\mu, v)=$ $\mathcal{N}\left(\boldsymbol{\varphi}_{v, \mu}\right)$. The constant $C$ depends only on $r_{1}, r_{2}, I_{1}$ and $I_{2}$, see Proposition 5.1 and 6.1 .

(ii) Furthermore, the perturbation $\boldsymbol{\xi}$ satisfies the equation of motion

$$
\begin{aligned}
\dot{\boldsymbol{\xi}}=J\left(L_{v, \mu} \boldsymbol{\xi}+\right. & \left.\mathcal{M}_{\boldsymbol{\varphi}_{v, \mu}}(\boldsymbol{\xi})+\left(V_{y}-V(y)\right) \boldsymbol{\xi}+R_{V} \boldsymbol{\varphi}_{v, \mu}\right) \\
& -\left((v-\dot{y}) \cdot \nabla_{x}\left(\boldsymbol{\varphi}_{v, \mu}+\boldsymbol{\xi}\right)+\dot{v} \cdot \nabla_{v} \boldsymbol{\varphi}_{v, \mu}+\dot{\mu} \partial_{\mu} \boldsymbol{\varphi}_{v, \mu}\right. \\
& \left.+(\mu-\dot{\vartheta}-V(y)) J\left(\boldsymbol{\varphi}_{v, \mu}+\boldsymbol{\xi}\right)-J x \cdot \nabla_{x} V(y) \boldsymbol{\varphi}_{v, \mu}\right)
\end{aligned}
$$

where $L_{v, \mu}$ is defined in (2.21),

$$
-\mathcal{M}_{\boldsymbol{\varphi}_{v, \mu}}(\boldsymbol{\xi})=\left(\frac{1}{|x|} *|\boldsymbol{\xi}|^{2}\right) \boldsymbol{\varphi}_{v, \mu}+\left(\frac{2}{|x|} *\left(\boldsymbol{\varphi}_{v, \mu} \cdot \boldsymbol{\xi}\right)\right) \boldsymbol{\xi}+\left(\frac{1}{|x|} *|\boldsymbol{\xi}|^{2}\right) \boldsymbol{\xi}
$$

and

$$
R_{V}(x):=V_{y}(x)-V(y)-x \cdot \nabla_{y} V(y),
$$

where $V_{y}(x)=V(x+y)$.

Remark 7.2. With the explicit form of $\Omega_{\boldsymbol{\varphi}_{v, \mu}}$ in Corollary 5.3 we rewrite Eq. (7.3) and (7.4) as

$$
\begin{array}{rr}
\gamma \dot{v}+\nabla_{y} V(y)=\mathcal{N}\left(\boldsymbol{\varphi}_{v, \mu}\right)^{-1}\left(Y_{4}, Y_{5}, Y_{6}\right)^{T} & \dot{\mu}-n_{, \mu}^{-1} n_{, v}^{T} \gamma^{-1} \nabla_{y} V(y)=Y_{8} \\
\dot{y}-v=-\left(Y_{1}, Y_{2}, Y_{3}\right) & \dot{\vartheta}-\mu+V(y)=-Y_{7},
\end{array}
$$

where $n:=\mathcal{N}\left(\boldsymbol{\varphi}_{v, \mu}\right), n_{, v_{j}}=\partial_{v_{j}} \mathcal{N}\left(\boldsymbol{\varphi}_{v, \mu}\right), n_{, \mu}=\partial_{\mu} \mathcal{N}\left(\boldsymbol{\varphi}_{v, \mu}\right), \tau_{j k}:=\left(\partial_{v_{j}} \boldsymbol{\varphi}_{v, \mu}, L_{v, \mu} \partial_{v_{k}} \boldsymbol{\varphi}_{v, \mu}\right)_{2}$ and $\gamma_{j k}:=n^{-1}\left(\tau_{j k}+n_{, \mu}^{-1} n_{, v_{j}} n_{, v_{k}}\right)$. See also Corollary 7.4 below.

Remark 7.3. The expression (7.6) is equivalent to (2.15) in the moving frame and with the decomposition (7.1) inserted. This equation does contain the information about Eqns. (7.9)-(7.10). We can of course remove this information from (7.6) by a 'projection'. But since we do not explicitly need this form of Eq. (7.6), we have refrained from writing out this expression.

Proof. By Proposition 7.5 and $\boldsymbol{\psi}_{0} \in U_{\delta}\left(\mathrm{Z}_{3}\right)$, the solution $\boldsymbol{\psi}$ to Eq. (2.15) satisfies $\boldsymbol{\psi} \in$ $U_{\delta}\left(\mathrm{Z}_{2}\right)$ for some positive times, and the decomposition of $\boldsymbol{\psi}$ into $(\zeta, \boldsymbol{\xi})$ exists and is 
unique. For such times we express (2.15) in terms of $\zeta, \boldsymbol{\xi}$, with $\zeta \in \mathrm{Z}_{1}$. First, we calculate the time derivative of (7.1):

$$
\begin{aligned}
d_{t} \boldsymbol{\psi}=\mathrm{e}^{-\vartheta J} & \left(-\dot{y} \cdot\left(\nabla_{x} \boldsymbol{\varphi}_{v, \mu}(x-y)+\nabla_{x} \boldsymbol{\xi}(x-y, t)\right)+\dot{v} \cdot \nabla_{v} \boldsymbol{\varphi}_{v, \mu}(x-y)\right. \\
& \left.+\dot{\mu} \partial_{\mu} \boldsymbol{\varphi}_{v, \mu}(x-y)-\dot{\vartheta} J\left(\boldsymbol{\varphi}_{v, \mu}(x-y)+\boldsymbol{\xi}(x-y, t)\right)+\partial_{t} \boldsymbol{\xi}(x-y, t)\right)
\end{aligned}
$$

We denote the decomposition of $\boldsymbol{\psi}$ inserted into the right-hand side of (2.15) by $A$. That is

$$
A:=J \mathcal{H}_{V}^{\prime}\left(\mathrm{e}^{-\vartheta J}\left(\boldsymbol{\varphi}_{v, \mu}(x-y)+\boldsymbol{\xi}(x-y, t)\right)\right) .
$$

We expand $\mathcal{H}_{V}^{\prime}$ around $\boldsymbol{\varphi}_{v, \mu}$, with the relations $\mathcal{E}_{v, \mu}^{\prime}\left(\boldsymbol{\varphi}_{v, \mu}\right)=\mathcal{H}_{V \equiv 0}^{\prime}\left(\boldsymbol{\varphi}_{v, \mu}\right)+\mu \varphi_{v, \mu}-v$. $\nabla_{x} J \boldsymbol{\varphi}_{v, \mu}, L_{v, \mu}:=\mathcal{E}_{v, \mu}^{\prime \prime}\left(\boldsymbol{\varphi}_{v, \mu}\right)$ (for its explicit form see $(2.21)$ ) and $\mathcal{E}_{v, \mu}^{\prime}\left(\boldsymbol{\varphi}_{\zeta}\right)=0$ we find

$$
\begin{array}{r}
A=\mathrm{e}^{-\vartheta J} J\left(L_{v, \mu} \boldsymbol{\xi}-\mu\left(\boldsymbol{\varphi}_{v, \mu}+\boldsymbol{\xi}\right)+v \cdot \nabla_{x} J\left(\boldsymbol{\varphi}_{v, \mu}+\boldsymbol{\xi}\right)+\mathcal{M}_{\boldsymbol{\varphi}_{v, \mu}}(\boldsymbol{\xi})\right)(x-y, t)+ \\
(V(x)-V(y)+V(y))\left(\boldsymbol{\varphi}_{v, \mu}(x-y)+\boldsymbol{\xi}(x-y, t)\right),
\end{array}
$$

where

$$
\mathcal{M}_{\boldsymbol{\varphi}_{v, \mu}}(\boldsymbol{\xi}):=\mathcal{H}_{V}^{\prime}\left(\boldsymbol{\varphi}_{v, \mu}+\boldsymbol{\xi}\right)-\mathcal{H}_{V}^{\prime}\left(\boldsymbol{\varphi}_{v, \mu}\right)-\mathcal{H}_{V}^{\prime \prime}\left(\boldsymbol{\varphi}_{v, \mu}\right) \boldsymbol{\xi}
$$

We insert the explicit form of $\mathcal{H}_{V}$ into the expression for $\mathcal{M}_{\varphi_{v, \mu}}$ above, simplification gives the result (7.7).

The expressions (7.11) and (7.13) are the right and left-hand side of (2.15). Both sides have a common phase which we cancel. Furthermore, both sides also have a common spacial translation $x \mapsto x-y(t)$, which we remove. That is, we consider the equation in a moving frame. Thus, we can rewrite (2.15) into the form

$$
\begin{aligned}
& (v-\dot{y}) \cdot \nabla_{x}\left(\boldsymbol{\varphi}_{v, \mu}+\boldsymbol{\xi}\right)+\dot{v} \cdot \nabla_{v} \boldsymbol{\varphi}_{v, \mu}+(\mu-\dot{\vartheta}-V(y)) J\left(\boldsymbol{\varphi}_{v, \mu}+\boldsymbol{\xi}\right)+\dot{\mu} \partial_{\mu} \boldsymbol{\varphi}_{v, \mu} \\
& \left.-\nabla_{y} V(y) \cdot x J \varphi_{v, \mu}+\partial_{t} \boldsymbol{\xi}=J\left(L_{v, \mu} \boldsymbol{\xi}+\mathcal{M}_{\boldsymbol{\varphi}_{v, \mu}}(\boldsymbol{\xi})+\left(V_{y}-V(y)\right) \boldsymbol{\xi}\right)+R_{V} \boldsymbol{\varphi}_{v, \mu}\right),
\end{aligned}
$$

after collecting terms of similar types. Here $V_{y}(x):=V(x+y)$, and $\boldsymbol{\varphi}_{v, \mu}$ and $\boldsymbol{\xi}$ are evaluated at $x$ and $x, t$ respectively. Furthermore, $R_{V}$ is defined as

$$
R_{V}(x):=V_{y}(x)-V(y)-x \cdot \nabla_{y} V(y) .
$$

Thus (7.15) is the desired equation (7.6) with the terms somewhat rearranged. We have showed part (ii) of the proposition. See also Remark 7.3.

To show part (i), let $\boldsymbol{z}_{k} \in \mathrm{T}_{\boldsymbol{\varphi}_{v, \mu}} \mathrm{M}_{1}$ where $\left\{\boldsymbol{z}_{k}\right\}$ are ordered as in (5.1), and apply the symplectic form $\omega\left(\boldsymbol{z}_{k}, \cdot\right)$ to $(7.15)$, then:

$$
\begin{aligned}
& \omega\left(\boldsymbol{z}_{k},(v-\dot{y}) \cdot \nabla_{x}\left(\boldsymbol{\varphi}_{v, \mu}+\boldsymbol{\xi}\right)+\right. \dot{v} \cdot \nabla_{v} \boldsymbol{\varphi}_{v, \mu}+(\mu-\dot{\vartheta}-V(y)) J\left(\boldsymbol{\varphi}_{v, \mu}+\boldsymbol{\xi}\right)+\dot{\mu} \partial_{\mu} \boldsymbol{\varphi}_{v, \mu} \\
&\left.-\nabla_{y} V(y) \cdot x J \boldsymbol{\varphi}_{v, \mu}+\partial_{t} \boldsymbol{\xi}\right) \\
&=\omega\left(\boldsymbol{z}_{k}, J L_{v, \mu} \boldsymbol{\xi}+J \mathcal{M}_{\boldsymbol{\varphi}_{v, \mu}}(\boldsymbol{\xi})+J\left(V_{y}-V(y)\right) \boldsymbol{\xi}+J R_{V} \boldsymbol{\varphi}_{v, \mu}\right)
\end{aligned}
$$

Denote the right-hand side of this equation with $B_{k}$, we claim that the term $\omega\left(\boldsymbol{z}_{k}, J L_{v, \mu} \boldsymbol{\xi}\right)$ vanishes for all $\boldsymbol{z}_{k} \in \mathrm{T}_{\boldsymbol{\varphi}_{v, \mu}} \mathrm{M}_{1}$. To show this claim, first note the identity $\omega\left(\boldsymbol{z}_{k}, J L_{v, \mu} \boldsymbol{\xi}\right)=$ 
$\left(L_{v, \mu} \boldsymbol{z}_{k}, \boldsymbol{\xi}\right)_{2}$, where we used that $L_{v, \mu}$ is symmetric. Secondly, $L_{v, \mu} \boldsymbol{z}_{k}$ either is zero, or $J \boldsymbol{z}_{k^{\prime}}$ for some $k^{\prime}$; see eqns. (4.4) and (4.5). In the first case, we have showed the claim, in the latter case recall that $\boldsymbol{\xi}$ satisfies the decomposition conditions (6.4). Thus we have $\omega\left(\boldsymbol{z}_{k^{\prime}}, \boldsymbol{\xi}\right)=0$ and we have shown the claim. The consequence is

$$
B_{k}=\omega\left(\boldsymbol{z}_{k}, J \mathcal{M}_{\boldsymbol{\varphi}_{v, \mu}}(\boldsymbol{\xi})+J\left(V_{y}-V(y)\right) \boldsymbol{\xi}+J R_{V} \boldsymbol{\varphi}_{v, \mu}\right) .
$$

To estimate the nonlinear term in $B_{k}$, we use the Hardy-Littlewood-Sobolev inequality [21] and a Sobolev embedding theorem. The remaining terms involve the potential and Taylor expansions of it, we bound these terms by using the fundamental theorem of calculus, and eqn. (3.1). We find

$$
B_{k} \leq C\left(\|\boldsymbol{\xi}\|_{\mathrm{H}^{\frac{1}{2}}}^{3}+\|\boldsymbol{\xi}\|_{\mathrm{H}^{\frac{1}{2}}}^{2}+\varepsilon^{2}\right) .
$$

We now return to (7.17), let the infinitesimal generators, $K$, and their coefficients, $\alpha$, be defined by

$$
K:=\left(\nabla_{x}, \nabla_{v}, J, \partial_{\mu}\right), \alpha:=(v-\dot{y}, \dot{v}, \mu-\dot{\vartheta}-V(y), \dot{\mu}) .
$$

We keep the above notation $B=\left(B_{1}, \ldots, B_{8}\right)$ to represent the right-hand side of (7.17), which by (7.19) is a perturbation for sufficiently small $\boldsymbol{\xi}$ and $\varepsilon$. With the observations that $\omega\left(\boldsymbol{z}_{k}, \nabla_{x} \boldsymbol{\xi}\right)=-\omega\left(\nabla_{x} \boldsymbol{z}_{k}, \boldsymbol{\xi}\right), \omega\left(\boldsymbol{z}_{k}, J \boldsymbol{\xi}\right)=-\omega\left(J \boldsymbol{z}_{k}, \boldsymbol{\xi}\right), \omega\left(\boldsymbol{z}_{k}, \boldsymbol{\xi}\right)=0$ and

$$
0=\partial_{t} \omega\left(\boldsymbol{z}_{k}, \boldsymbol{\xi}\right)=\dot{v} \cdot \omega\left(\nabla_{v} \boldsymbol{z}_{k}, \boldsymbol{\xi}\right)+\dot{\mu} \omega\left(\partial_{\mu} \boldsymbol{z}_{k}, \boldsymbol{\xi}\right)+\omega\left(\boldsymbol{z}_{k}, \boldsymbol{\xi}\right) .
$$

we re-write (7.17) as

$$
\sum_{j}\left(\left(\Omega_{\boldsymbol{\varphi}_{v, \mu}}\right)_{k j}-\omega\left(K_{j} \boldsymbol{z}_{k}, \boldsymbol{\xi}\right)\right) \alpha_{j}-\omega\left(\boldsymbol{z}_{k}, \nabla_{y} V(y) \cdot x J \boldsymbol{\varphi}_{v, \mu}\right)=B_{k},
$$

where $\left(\Omega_{\boldsymbol{\varphi}_{v, \mu}}\right)_{k j}=\omega\left(\boldsymbol{z}_{k}, K_{j} \boldsymbol{\varphi}_{v, \mu}\right)$ is as in Corollary 5.3. Solving for the leading term in $\alpha$ we find

$$
\alpha_{j}-\sum_{k}\left(\Omega_{\boldsymbol{\varphi}_{v, \mu}}\right)_{j k}^{-1} \omega\left(\boldsymbol{z}_{k}, \nabla_{y} V(y) \cdot x J \boldsymbol{\varphi}_{v, \mu}\right)=\sum_{k}\left(\Omega_{\boldsymbol{\varphi}_{v, \mu}}\right)_{j k}^{-1}\left(B_{k}+\sum_{l} \omega\left(K_{l} \boldsymbol{z}_{k}, \boldsymbol{\xi}\right) \alpha_{l}\right) .
$$

Denote the right-hand side with $Y_{j}$, then the uniform lower bound on $\Omega_{\varphi_{v, \mu}}$, given by Proposition 5.1, yields that

$$
|Y| \leq C\left(\varepsilon^{2}+\|\boldsymbol{\xi}\|_{2}|\alpha|+\|\boldsymbol{\xi}\|_{\mathrm{H}^{\frac{1}{2}}}^{2}+\|\boldsymbol{\xi}\|_{\mathrm{H}^{\frac{1}{2}}}^{3}\right) .
$$

and $|\alpha| \leq C \varepsilon+|Y|$. The constant $C$, in both cases, depends only on $r_{1}, r_{2}, I_{1}$ and $I_{2}$. Since $\boldsymbol{z}_{k}=K_{k} \boldsymbol{\varphi}_{v, \mu}$ we find that

$$
\omega\left(\boldsymbol{z}_{k}, x \cdot \nabla_{y} V(y)\right)=-\delta_{k \nu} \nabla_{y_{\nu}} V(y) \mathcal{N}\left(\boldsymbol{\varphi}_{v, \mu}\right),
$$

for $\nu=1,2,3$. Thus

$$
\alpha_{j}+\mathcal{N}\left(\boldsymbol{\varphi}_{v, \mu}\right) \sum_{\nu=1}^{3}\left(\Omega_{\boldsymbol{\varphi}_{v, \mu}}^{-1}\right)_{j \nu} \nabla_{y_{\nu}} V(y)=Y_{j}
$$

and we have proved the proposition. 
We have derived a set of ordinary differential equations (7.3). The right-hand side remains small by the main theorem, provided the decomposition exists. Standard ODE theory shows that the solution to (7.3) is well defined as long as the decomposition is well defined. This agrees with what we expect from the global well-posedness of the solution $\boldsymbol{\psi}$ to $(2.15)$.

We close this section with yet another form of (7.9):

Corollary 7.4. With the change of variables $(\mu, v) \mapsto(\mathcal{N}, \mathcal{P})$, through $\mathcal{N}=\mathcal{N}\left(\boldsymbol{\varphi}_{v, \mu}\right)$ and $\mathcal{P}=\mathcal{P}\left(\boldsymbol{\varphi}_{v, \mu}\right)$, defined in (2.13) and (2.14), the equations for $\dot{v}$ and $\dot{\mu}$, (Eqns.(7.9)) take the form

$$
\begin{aligned}
d_{t} \mathcal{P}+\mathcal{N} \nabla_{y} V(y) & =\left(X_{1}, X_{2}, X_{3}\right)^{T}, \\
d_{t} \mathcal{N} & =X_{8},
\end{aligned}
$$

where each of $X_{j}, j=1,2,3,8$ are related to $Y_{j}$ above by $X_{j}=\sum_{k}\left(\Omega_{\varphi_{v, \mu}}\right)_{j k} Y_{k}$.

Proof. Insert $\boldsymbol{z}_{k}=\left(\nabla_{x} \boldsymbol{\varphi}_{v, \mu}, J \boldsymbol{\varphi}_{v, \mu}\right)$ into (7.17) and simplify to obtain

$$
\begin{aligned}
d_{t} \mathcal{P}\left(\boldsymbol{\varphi}_{v, \mu}\right)+\mathcal{N}\left(\boldsymbol{\varphi}_{v, \mu}\right) \nabla_{y} V(y) & =\left(X_{1}, X_{2}, X_{3}\right)^{T} \\
d_{t} \mathcal{N}\left(\boldsymbol{\varphi}_{v, \mu}\right) & =X_{8}
\end{aligned}
$$

where

$$
X_{j}=\omega\left(K_{j} \boldsymbol{\varphi}_{v, \mu}, J\left(\mathcal{M}_{\boldsymbol{\varphi}_{v, \mu}}(\boldsymbol{\xi})+\left(V_{y}-V(y)\right) \boldsymbol{\xi}+R_{V} \boldsymbol{\varphi}_{v, \mu}\right)\right)+\sum_{k=1}^{8} \alpha_{k} \omega\left(K_{k} K_{j} \boldsymbol{\varphi}_{v, \mu}, \boldsymbol{\xi}\right),
$$

in which $K$ is defined by (7.20). The change of variables $(\mu, v) \mapsto(\mathcal{N}, \mathcal{P})$ gives $(7.27)$ and (7.28). To see the relation between $X$ and $Y$, see equation (7.23).

Proposition 7.5. If $\boldsymbol{\psi}_{0} \in \tilde{\Gamma}$, then the solution of (1.1) satisfies

$$
\psi \in \mathrm{C}^{0}([0, T) ; \tilde{\Gamma})
$$

where $T \in(0, \infty]$ is the maximal time of existence. Furthermore, we have that $T=\infty$ holds whenever $\mathcal{N}(\boldsymbol{\psi})<N_{\mathrm{c}}$, for some universal constant $N_{\mathrm{c}}>2 / \pi$.

Proof. For $\tilde{\Gamma} \subset \mathrm{H}^{\frac{1}{2}}$ replaced by $\mathrm{H}^{\frac{1}{2}}$, the claim follows from the well-posedness results for (1.1) proven in [20] (where also more general $V$ 's are treated).

It remains to show that $t \mapsto|x|^{1 / 2} \psi(t)$ is a continuous map from $[0, T)$ into $\mathrm{L}^{2}$. First, we notice that $\boldsymbol{\psi}_{0} \in \tilde{\Gamma}$ implies that $\boldsymbol{\psi}(t) \in \tilde{\Gamma}$ for all $0 \leq t<T$. This claim follows in particular from a direct adaption of [12][Lemma A], yielding the formula

$$
(\boldsymbol{\psi}(t),|x| \boldsymbol{\psi}(t))_{2}=(\boldsymbol{\psi}(0),|x| \boldsymbol{\psi}(t))_{2}+\int_{0}^{t}\left(\boldsymbol{\psi}(s), J\left[|x|, \sqrt{-\Delta+m^{2}}\right] \boldsymbol{\psi}(s)\right)_{2} \mathrm{~d} s .
$$

Here the commutator $\left[|x|, \sqrt{-\Delta+m^{2}}\right]$ is a bounded operator on $\mathrm{L}^{2}$; see also [12] for this. 
Moreover, equation (7.32) shows in particular that $t \mapsto\left\||x|^{1 / 2} \boldsymbol{\psi}(t)\right\|_{2}$ is continuous. Assume now that $t_{n} \rightarrow t_{*} \in[0, T)$ is a sequence of times. Then $u_{n}:=|x|^{1 / 2} \boldsymbol{\psi}\left(t_{n}\right)$ is a bounded sequence in $\mathrm{L}^{2}$. By possibly passing to a subsequence, we have that $u_{n}$ converges weakly to some $u_{*}$ with $\liminf _{n \rightarrow \infty}\left\|u_{n}\right\|_{2} \geq\left\|u_{*}\right\|_{2}$. But since $t \mapsto\left\||x|^{1 / 2} \boldsymbol{\psi}(t)\right\|_{2}$ is continuous, we have that $\lim _{n \rightarrow \infty}\left\|u_{n}\right\|_{2}=\left\|u_{*}\right\|_{2}$ holds. Thus we conclude that $u_{n}$ actually converges strongly to $u_{*}$ in $\mathrm{L}^{2}$, showing that $t \mapsto|x|^{1 / 2} \boldsymbol{\psi}(t)$ is a continuous map from $[0, T)$ into $\mathrm{L}^{2}$.

\section{Weighted dynamics}

Let $\boldsymbol{\psi}$ be a solution to (2.15) with initial condition $\boldsymbol{\psi}_{0} \in U_{\varepsilon}\left(\mathrm{Z}_{3}\right)$. Then, under Assumption 2.1, there is, for some positive time, a unique decomposition of $\boldsymbol{\psi}$ into $\zeta, \boldsymbol{\xi}$ (Proposition 6.1). Furthermore, if we add that $V$ satisfies (3.1) for some $\varepsilon>0$, we find the equations of motion for $\boldsymbol{\xi}$ in Proposition 7.1. Let $\mathcal{Q}_{\delta_{0}}(t):=\left(\boldsymbol{\xi}(\cdot, t)|x| \mathrm{e}^{-\delta_{0}|x|}, \boldsymbol{\xi}(\cdot, t)\right)_{2}$. In this section we use the equations of motions for $\boldsymbol{\xi}$ to show that the weighted expectation value $\mathcal{Q}(t)=\mathcal{Q}_{\delta_{0}=0}(t)$ is well defined and small.

We have the result:

Proposition 8.1. Let $U_{\varepsilon}\left(\mathrm{Z}_{3}\right)$ be defined as above. Let the Assumption 2.1 be satisfied, and let $\boldsymbol{\psi}$ be a solution to (2.15) with initial condition $\boldsymbol{\psi}_{0} \in U_{\varepsilon}\left(\mathrm{Z}_{3}\right)$. Denote its decomposition by $(\zeta, \boldsymbol{\xi})$. Let the external potential $V$ satisfy (3.1), for some small parameter $\varepsilon>0$. Then, for times such that the decomposition is unique there is a constant $0<c<\infty$ depends only on $r_{j}, I_{j}, j=1,2,3$ such that

$$
\begin{aligned}
\sup _{s \leq t} \mathcal{Q}_{\delta_{0}}(s) \leq \mathcal{Q}_{\delta_{0}}(0)+c t \sup _{s \leq t}\left(\mathcal{Q}_{\delta_{0}}(s)\left(\|\boldsymbol{\xi}(s)\|_{\mathrm{H}^{\frac{1}{2}}}+\|\boldsymbol{\xi}(s)\|_{\mathrm{H}^{\frac{1}{2}}}^{2}\right)\right. & \\
& \left.+(\varepsilon+|\alpha(s)|)\|\boldsymbol{\xi}(s)\|_{2}+\|\boldsymbol{\xi}(s)\|_{\mathrm{H}^{\frac{1}{2}}}^{2}+\|\boldsymbol{\xi}(s)\|_{\mathrm{H}^{\frac{1}{2}}}^{3}\right),
\end{aligned}
$$

where $\alpha$ is defined in (7.4).

Let $\mathcal{Q}(t):=(\boldsymbol{\xi}(\cdot, t)|x|, \boldsymbol{\xi}(\cdot, t))_{2}$. From the above result and the assumption on the initial condition in the main Theorem 3.1 we have

Corollary 8.2. Assume, in addition to the assumptions of Proposition 8.1, that $\| \boldsymbol{\psi}_{0}-$ $\boldsymbol{\varphi}_{\zeta_{0}} \|_{\tilde{\Gamma}} \leq \varepsilon<\delta$. Then there is a constant $0<C<\infty$ depending only on $I_{j}$ and $r_{j}$ for $j=1,2,3$ such that

$$
\sup _{s \leq t} \mathcal{Q}(s) \leq C \varepsilon+\sup _{s \leq t}\|\boldsymbol{\xi}(s)\|_{\mathrm{H}^{\frac{1}{2}}}
$$

for positive times $t$ such that $\boldsymbol{\psi} \in U_{\delta}\left(\mathrm{Z}_{1}\right)$ and such that time, $t$, satisfies the inequality

$$
t \leq \frac{1}{2 c} \frac{1}{\varepsilon+\sup _{s \leq t}\left(|\alpha(s)|+\|\boldsymbol{\xi}(\cdot, s)\|_{\mathrm{H}^{\frac{1}{2}}}+\|\boldsymbol{\xi}(\cdot, s)\|_{\mathrm{H}^{\frac{1}{2}}}^{2}\right)}
$$

with constant c as in Proposition 8.1. 
Proof of Corollary 8.2. By assumption $\left\|\boldsymbol{\psi}_{0}-\boldsymbol{\varphi}_{\zeta_{0}}\right\|_{\tilde{\Gamma}} \leq \varepsilon$, thus by the definition of $\|\cdot\|_{\tilde{\Gamma}}$, $\left\|\left(\varepsilon|x|^{1 / 2}\right)\left(\boldsymbol{\psi}_{0}-\boldsymbol{\varphi}_{\zeta_{0}}\right)\right\|_{2} \leq \varepsilon$. Hence

$$
\left\|\boldsymbol{\psi}_{0}-\boldsymbol{\varphi}_{\varsigma\left(\boldsymbol{\psi}_{0}\right)}\right\|_{\tilde{\Gamma}} \leq \varepsilon+\left\|\boldsymbol{\varphi}_{\varsigma}\left(\boldsymbol{\psi}_{0}\right)-\boldsymbol{\varphi}_{\zeta_{0}}\right\|_{\tilde{\Gamma}}
$$

Part (ii) in Proposition 6.1 yields that $\left\|\boldsymbol{\varphi}_{\zeta_{0}}-\boldsymbol{\varphi}_{\varsigma}\left(\boldsymbol{\psi}_{0}\right)\right\|_{\tilde{\Gamma}} \leq C \varepsilon$. Thus $\mathcal{Q}_{\delta_{0}}(0) \leq \mathcal{Q}(0) \leq C \varepsilon$.

As we consider times, the decomposition time, such that $\boldsymbol{\psi} \in U_{\delta}\left(\mathrm{Z}_{2}\right)$ (and hence $\zeta(t) \in \mathrm{Z}_{1}$ ), we have that the result in Proposition 8.1 holds. Let $t$ be such that it is smaller than the minimum of the decomposition time and the times such that (8.3) holds. For such times, estimate (8.1) simplifies to

$$
\sup _{s \leq t} \mathcal{Q}_{\delta_{0}}(s) \leq C \varepsilon+\frac{1}{2}\|\boldsymbol{\xi}\|_{\mathrm{H}^{\frac{1}{2}}}
$$

The right-hand side is independent of $\delta_{0}$, we can thus take the limit to find the result (8.2).

Proof of Proposition 8.1. Notice that

$$
\mathcal{Q}_{\delta_{0}}(t)=\mathcal{Q}_{\delta_{0}}(0)+\int_{0}^{t} d_{s} \mathcal{Q}_{\delta_{0}}(s) \mathrm{d} s .
$$

Since $\mathcal{Q}_{\delta_{0}}$ is positive for all times, we find

$$
\mathcal{Q}_{\delta_{0}}(t) \leq \mathcal{Q}_{\delta_{0}}(0)+t \sup _{s \leq t}\left|d_{s} \mathcal{Q}_{\delta_{0}}(s)\right| .
$$

The right-hand side is independent under the map $t \rightarrow s, \sum_{s \leq t}$. Thus we find

$$
\sup _{s \leq t} \mathcal{Q}_{\delta_{0}}(s) \leq \mathcal{Q}_{\delta_{0}}(0)+t \sup _{s \leq t}\left|d_{s} \mathcal{Q}_{\delta_{0}}(s)\right|
$$

To bound this we need to estimate $d_{s} \mathcal{Q}_{\delta_{0}}(s)$. As mentioned in the introduction to this section we have assumed $\psi_{0} \in U_{\varepsilon}\left(\mathrm{Z}_{3}\right)$, thus for some times (to be determined) $\boldsymbol{\psi} \in U_{\delta}\left(\mathrm{Z}_{2}\right)$. For such $\boldsymbol{\psi}$, there is, under Assumption 2.1 and by Proposition 6.1, a unique decomposition of $\boldsymbol{\psi} \mapsto(\boldsymbol{\xi}, \zeta)$. Proposition 7.1 yields the equation of motion for $\boldsymbol{\xi}$ in (7.6), that is

$$
\begin{aligned}
\partial_{t} \boldsymbol{\xi}=J\left(L_{v, \mu} \boldsymbol{\xi}+\mathcal{M}_{\boldsymbol{\varphi}_{v, \mu}}(\boldsymbol{\xi})\right. & \left.+\left(V_{y}-V(y)\right)\left(\boldsymbol{\xi}+\boldsymbol{\varphi}_{v, \mu}\right)\right)+(\dot{y}-v) \cdot \nabla\left(\boldsymbol{\varphi}_{v, \mu}+\boldsymbol{\xi}\right) \\
& -\dot{v} \cdot \nabla_{v} \boldsymbol{\varphi}_{v, \mu}-\dot{\mu} \partial_{\mu} \boldsymbol{\varphi}_{v, \mu}-(\mu-\dot{\vartheta}-V(y)) J\left(\boldsymbol{\varphi}_{v, \mu}+\boldsymbol{\xi}\right),
\end{aligned}
$$

where $L_{v, \mu}$ is the $4 \times 4$ matrix operator introduced in (2.21). We repeat the explicit form of $L_{v, \mu}$ for clarity.

$$
\begin{aligned}
L_{11} \xi_{1} & :=\sqrt{-\Delta+m^{2}} \xi_{1}+(-m+\mu) \xi_{1}-\frac{1}{|x|} *\left|\boldsymbol{\varphi}_{v, \mu}\right|^{2} \xi_{1}-\left(\frac{2}{|x|} *\left(\xi_{1} \varphi_{v, \mu}^{(1)}\right)\right) \varphi_{v, \mu}^{(1)}, \\
L_{12} \xi_{2} & :=v \cdot \nabla \xi_{2}-\left(\frac{2}{|x|} *\left(\xi_{2} \varphi_{v, \mu}^{(2)}\right)\right) \varphi_{v, \mu}^{(1)}, \\
L_{21} \xi_{1} & :=-v \cdot \nabla \xi_{1}-\left(\frac{2}{|x|} *\left(\xi_{1} \varphi_{v, \mu}^{(1)}\right)\right) \varphi_{v, \mu}^{(2)}, \\
L_{22} \xi_{2} & :=\sqrt{-\Delta+m^{2}} \xi_{2}+(-m+\mu) \xi_{2}-\left(\frac{1}{|x|} *\left|\boldsymbol{\varphi}_{v, \mu}\right|^{2}\right) \xi_{2}-\left(\frac{2}{|x|} *\left(\xi_{2} \varphi_{v, \mu}^{(2)}\right)\right) \varphi_{v, \mu}^{(2)},
\end{aligned}
$$


where we have used the notation $\varphi_{v, \mu}=\left(\varphi_{v, \mu}^{(1)}, \varphi_{v, \mu}^{(2)}\right)^{T}$. We regularize $|x|$ by

$$
f_{\delta_{0}}(x):=|x| \mathrm{e}^{-\delta_{0}|x|}
$$

This is a bounded function, and $2 \mathcal{Q}_{\delta_{0}}:=\left(\boldsymbol{\xi}, f_{\delta_{0}} \boldsymbol{\xi}\right)_{2}$ is well defined, since $\boldsymbol{\xi} \in \mathrm{L}^{2}$ and $f_{\delta_{0}} \in \mathrm{L}^{\infty}$.

The time derivative of $\mathcal{Q}_{\delta_{0}}$ can be expressed as

$$
2 d_{t} \mathcal{Q}_{\delta_{0}}=\left(\partial_{t} \boldsymbol{\xi}, f_{\delta_{0}} \boldsymbol{\xi}\right)_{2}+\left(\boldsymbol{\xi}, f_{\delta_{0}} \partial_{t} \boldsymbol{\xi}\right)_{2}=2\left(\partial_{t} \boldsymbol{\xi}, f_{\delta_{0}} \boldsymbol{\xi}\right)_{2}
$$

Inserting the above equation for $\boldsymbol{\xi}$, we find

$$
\begin{aligned}
& d_{t} \mathcal{Q}_{\delta_{0}}=(\left.J\left(L_{v, \mu} \boldsymbol{\xi}+\mathcal{M}_{\boldsymbol{\varphi}_{v, \mu}}(\boldsymbol{\xi})+\left(V_{y}-V(y)\right)\left(\boldsymbol{\xi}+\boldsymbol{\varphi}_{v, \mu}\right)\right), f_{\delta_{0}} \boldsymbol{\xi}\right)_{2} \\
&+\left(-\dot{v} \cdot \nabla_{v} \boldsymbol{\varphi}_{v, \mu}-\dot{\mu} \partial_{\mu} \boldsymbol{\varphi}_{v, \mu}-(\mu-\dot{\vartheta}-V(y)) J\left(\boldsymbol{\varphi}_{v, \mu}+\boldsymbol{\xi}\right), f_{\delta_{0}} \boldsymbol{\xi}\right)_{2} \\
&+\left((\dot{y}-v) \cdot \nabla\left(\boldsymbol{\varphi}_{v, \mu}+\boldsymbol{\xi}\right), f_{\delta_{0}} \boldsymbol{\xi}\right)_{2}
\end{aligned}
$$

To simplify this expression, we note that $(J a, r(x) a)_{2}=0$ for all bounded scalar functions $r(x)$. Similarly $2(\nabla a, r a)_{2}=-(a, a \nabla r)_{2}$, for any scalar function $r(x)$. Thus

$$
\begin{aligned}
d_{t} \mathcal{Q}_{\delta_{0}}=\left(J\left(L_{v, \mu} \boldsymbol{\xi}+\mathcal{M}_{\boldsymbol{\varphi}_{v, \mu}}(\boldsymbol{\xi})\right), f_{\delta_{0}} \boldsymbol{\xi}\right)_{2}+\left(J\left(V_{y}-V(y)\right) \boldsymbol{\varphi}_{v, \mu}, f_{\delta_{0}} \boldsymbol{\xi}\right)_{2}+ \\
(\dot{y}-v) \cdot\left(\left(\nabla \boldsymbol{\varphi}_{v, \mu}, f_{\delta_{0}} \boldsymbol{\xi}\right)_{2}-\frac{1}{2}\left(\boldsymbol{\xi}, \boldsymbol{\xi} \nabla f_{\delta_{0}}\right)_{2}\right) \\
-(\mu-\dot{\vartheta}-V(y))\left(J \boldsymbol{\varphi}_{v, \mu}, f_{\delta_{0}} \boldsymbol{\xi}\right)_{2}-\left(\dot{v} \cdot \nabla_{v} \boldsymbol{\varphi}_{v, \mu}+\dot{\mu} \partial_{\mu} \boldsymbol{\varphi}_{v, \mu}, f_{\delta_{0}} \boldsymbol{\xi}\right)_{2}
\end{aligned}
$$

To estimate $d_{t} \mathcal{Q}_{\delta_{0}}$, we begin with recalling the definition of $\alpha$ in eqn. (7.4), thus all the terms terms $\dot{y}-v, \dot{v}$, etc. are bounded by $|\alpha|$. Furthermore, we note that $\left|\nabla f_{\delta_{0}}\right| \leq 1$. Thus

$$
\begin{aligned}
& \left|d_{t} \mathcal{Q}_{\delta_{0}}\right| \leq\left|\left(J L_{v, \mu} \boldsymbol{\xi}, f_{\delta_{0}} \boldsymbol{\xi}\right)_{2}\right|+\left|\left(\mathcal{M}_{\boldsymbol{\varphi}_{v, \mu}}(\boldsymbol{\xi}), f_{\delta_{0}} \boldsymbol{\xi}\right)_{2}\right|+C \varepsilon\left\||x| f_{\delta_{0}} \boldsymbol{\varphi}_{v, \mu}\right\|_{2}\|\boldsymbol{\xi}\|_{2} \\
& +|\alpha|\left(\left\|f_{\delta_{0}} \nabla \boldsymbol{\varphi}_{v, \mu}\right\|_{2}+\left\|f_{\delta_{0}} J \boldsymbol{\varphi}_{v, \mu}\right\|_{2}+\left\|f_{\delta_{0}} \partial_{\mu} \boldsymbol{\varphi}_{v, \mu}\right\|_{2}+\left\|f_{\delta_{0}} \nabla_{v} \boldsymbol{\varphi}_{v, \mu}\right\|_{2}\right)\|\boldsymbol{\xi}\|_{2}+\frac{1}{2}\|\boldsymbol{\xi}\|_{2}^{2}
\end{aligned}
$$

By Proposition 4.3, we know that all terms of the form $\left\|f_{\delta_{0}} \boldsymbol{z}_{j}\right\|_{2}$ satisfy $\left\|f_{\delta_{0}} \boldsymbol{z}_{j}\right\|_{2} \leq$ $\left\||x| \boldsymbol{z}_{j}\right\|_{2} \leq C$, where $\boldsymbol{z}_{j}$ is of the form $K \boldsymbol{\varphi}_{v, \mu}$ and $K \in\left\{|x|, \nabla_{x}, J, \partial_{\mu}, \nabla_{v}\right\}$, and $C$ being independent of $\delta_{0}$. Hence

$$
\left|d_{t} \mathcal{Q}_{\delta_{0}}\right| \leq\left|\left(J L_{v, \mu} \boldsymbol{\xi}, f_{\delta_{0}} \boldsymbol{\xi}\right)_{2}\right|+\left|\left(J \mathcal{M}_{\boldsymbol{\varphi}_{v, \mu}}(\boldsymbol{\xi}), f_{\delta_{0}} \boldsymbol{\xi}\right)_{2}\right|+C(\varepsilon+|\alpha|)\|\boldsymbol{\xi}\|_{2}+\frac{1}{2}\|\boldsymbol{\xi}\|_{2}^{2}
$$

To estimate the term $\left(L_{v, \mu} \boldsymbol{\xi}, f_{\delta_{0}} \boldsymbol{\xi}\right)_{2}$, we write down this expression in detail

$$
\begin{aligned}
\left(J L_{v, \mu} \boldsymbol{\xi}, f_{\delta_{0}} \boldsymbol{\xi}\right)_{2}= & \left(\left(\begin{array}{cc}
0 & 1 \\
-1 & 0
\end{array}\right)\left(\begin{array}{cc}
L_{11} & L_{12} \\
L_{21} & L_{22}
\end{array}\right) \boldsymbol{\xi}, f_{\delta_{0}} \boldsymbol{\xi}\right)_{2}=\left(\left(\begin{array}{cc}
L_{21} & L_{22} \\
-L_{11} & -L_{12}
\end{array}\right) \boldsymbol{\xi}, f_{\delta_{0}} \boldsymbol{\xi}\right)_{2} \\
& =\left(L_{21} \xi^{(1)}+L_{22} \xi^{(2)}, f_{\delta_{0}} \xi^{(1)}\right)_{2}-\left(L_{11} \xi^{(1)}+L_{12} \xi^{(2)}, f_{\delta_{0}} \xi^{(2)}\right)_{2}
\end{aligned}
$$


Inserting the explicit expressions for the operator $L_{v, \mu}$ yields

$$
\begin{aligned}
&\left(L_{12} \xi^{(2)}, f_{\delta_{0}} \xi^{(2)}\right)_{2}=(v\left.\cdot \nabla \xi^{(2)}, f_{\delta_{0}} \xi^{(2)}\right)_{2}-\left(\frac{2}{|x|} *\left(\varphi_{v, \mu}^{(2)} \xi^{(2)}\right) \varphi_{v, \mu}^{(1)}, f_{\delta_{0}} \xi^{(2)}\right)_{2} \\
&=-\frac{v}{2} \cdot\left(\xi^{(2)}, \xi^{(2)} \nabla f_{\delta_{0}}\right)_{2}-\left(\frac{2}{|x|} *\left(\varphi_{v, \mu}^{(2)} \xi^{(2)}\right), f_{\delta_{0}} \varphi_{v, \mu}^{(1)} \xi^{(2)}\right)_{2}
\end{aligned}
$$

Once again we observe that $\sup _{x}\left|\nabla f_{\delta_{0}}\right| \leq 1$ holds, independent of $\delta_{0}$. Using the HardyLittlewood-Sobolev inequality we thus find

$$
\begin{aligned}
\left|\left(L_{12} \xi^{(2)}, f_{\delta_{0}} \xi^{(2)}\right)_{2}\right| \leq 2|v|\left\|\xi^{(2)}\right\|_{2}^{2}+C\left\|\varphi_{v, \mu}^{(2)} \xi^{(2)}\right\|_{12 / 5}\left\|f_{\delta_{0}} \varphi_{v, \mu}^{(1)} \xi^{(2)}\right\|_{12 / 5} \\
\leq 2|v|\left\|\xi^{(2)}\right\|_{2}^{2}+C\left\|\xi^{(2)}\right\|_{12 / 5}^{2}\left(\left\|\varphi_{v, \mu}^{(2)}\right\|_{\infty}^{12 / 5}\left\|f_{\delta_{0}} \varphi_{v, \mu}^{(1)}\right\|_{\infty}^{12 / 5}\right)^{5 / 12}
\end{aligned}
$$

Observe that $f_{\delta_{0}} \leq|x|, \sup \| x\left|\boldsymbol{\varphi}_{v, \mu}\right|<C$ and that $\|\boldsymbol{\xi}\|_{12 / 5} \leq C\|\boldsymbol{\xi}\|_{\mathrm{H}^{\frac{1}{2}}}$. We find that

$$
\left|\left(L_{12} \xi^{(2)}, f_{\delta_{0}} \xi^{(2)}\right)_{2}\right| \leq 2|v|\left\|\xi^{(2)}\right\|_{2}^{2}+C\left\|\xi^{(2)}\right\|_{\mathrm{H}^{\frac{1}{2}}}^{2}
$$

For the $L_{21}$-term we have

$$
\begin{aligned}
\left(L_{21} \xi^{(1)}, f_{\delta_{0}} \xi^{(1)}\right)_{2}=(-v & \left.\cdot \nabla \xi^{(1)}, f_{\delta_{0}} \xi^{(1)}\right)_{2}-\left(\frac{2}{|x|} *\left(\varphi_{v, \mu}^{(1)} \xi^{(1)}\right) \varphi_{v, \mu}^{(2)}, f_{\delta_{0}} \xi^{(1)}\right)_{2} \\
= & \frac{v}{2} \cdot\left(\xi^{(1)}, \xi^{(1)} \nabla f_{\delta_{0}}\right)_{2}-\left(\frac{2}{|x|} *\left(\varphi_{v, \mu}^{(1)} \xi^{(1)}\right), f_{\delta_{0}} \varphi_{v, \mu}^{(2)} \xi^{(1)}\right)_{2}
\end{aligned}
$$

Similar to the estimate of $L_{12}$, we obtain that

$$
\left|\left(L_{21} \xi^{(1)}, f_{\delta_{0}} \xi^{(1)}\right)_{2}\right| \leq 2|v|\left\|\xi^{(1)}\right\|_{2}^{2}+C\left\|\xi^{(1)}\right\|_{\mathrm{H}^{\frac{1}{2}}}^{2}
$$

The last two terms yield

$$
\begin{gathered}
\left(L_{22} \xi^{(2)}, f_{\delta_{0}} \xi^{(1)}\right)_{2}-\left(L_{11} \xi^{(1)}, f_{\delta_{0}} \xi^{(2)}\right)_{2}= \\
\left(\sqrt{-\Delta+m^{2}} \xi^{(2)}+(-m+\mu) \xi^{(2)}-\left(\frac{1}{|x|} *\left|\boldsymbol{\varphi}_{v, \mu}\right|^{2}\right) \xi^{(2)}-\left(\frac{2}{|x|} *\left(\xi^{(2)} \varphi_{v, \mu}^{(2)}\right)\right) \varphi_{v, \mu}^{(2)}, f_{\delta_{0}} \xi^{(1)}\right)_{2} \\
-\left(\sqrt{-\Delta+m^{2}} \xi^{(1)}+(-m+\mu) \xi^{(1)}-\left(\frac{1}{|x|} *\left|\boldsymbol{\varphi}_{v, \mu}\right|^{2}\right) \xi^{(1)}-\left(\frac{2}{|x|} *\left(\xi^{(1)} \varphi_{v, \mu}^{(1)}\right)\right) \varphi_{v, \mu}^{(1)}, f_{\delta_{0}} \xi^{(2)}\right)_{2} \\
=\left(\left[f_{\delta_{0}}, \sqrt{-\Delta+m^{2}}\right] \xi^{(2)}, \xi^{(1)}\right)_{2}-\left(\left(\frac{2}{|x|} *\left(\xi^{(2)} \varphi_{v, \mu}^{(2)}\right)\right) \varphi_{v, \mu}^{(2)}, f_{\delta_{0}} \xi^{(1)}\right)_{2} \\
\quad+\left(\left(\frac{2}{|x|} *\left(\xi^{(1)} \varphi_{v, \mu}^{(1)}\right)\right) \varphi_{v, \mu}^{(1)}, f_{\delta_{0}} \xi^{(2)}\right)_{2}, \quad(8.26)
\end{gathered}
$$

where $[A, B]=A B-B A$. The last two terms are both bounded by $C\|\boldsymbol{\xi}\|_{\mathrm{H}^{\frac{1}{2}}}^{2}$, analogous to the estimate for $L_{12}$. For the first term we use [9, Lemma A.3], see also Stein [29], that shows

$$
\left|\left(\left[f_{\delta_{0}}, \sqrt{-\Delta+m^{2}}\right] \xi^{(2)}, \xi^{(1)}\right)_{2}\right| \leq C\|\boldsymbol{\xi}\|_{2}^{2}
$$


with $C$ independent of $\delta_{0}$. Thus we find, using that $|v| \leq 1$,

$$
\left|\left(J L_{v, \mu} \boldsymbol{\xi}, f_{\delta_{0}} \boldsymbol{\xi}\right)_{2}\right| \leq C\|\boldsymbol{\xi}\|_{\mathrm{H}^{\frac{1}{2}}}^{2}
$$

The last term to estimate is $\left(\mathcal{M}_{\boldsymbol{\varphi}_{v, \mu}}(\boldsymbol{\xi}), f_{\delta_{0}} \boldsymbol{\xi}\right)_{2}$. To this end, we recall from Proposition 7.1 that

$$
-\mathcal{M}_{\boldsymbol{\varphi}_{v, \mu}}(\boldsymbol{\xi})=\frac{1}{|x|} *|\boldsymbol{\xi}|^{2} \boldsymbol{\varphi}_{v, \mu}+\frac{2}{|x|} *\left(\boldsymbol{\varphi}_{v, \mu} \cdot \boldsymbol{\xi}\right) \boldsymbol{\xi}+\frac{1}{|x|} *|\boldsymbol{\xi}|^{2} \boldsymbol{\xi}
$$

In this case we cannot use the Hardy-Littlewood-Sobolev estimate. But instead we can use the Kato $[17, \S \mathrm{V} .5 .4$, eq. (5.33)] inequality:

$$
\int_{\mathbb{R}^{3}} \frac{1}{|y|}|\boldsymbol{\xi}(y) \cdot \boldsymbol{u}(y)| \mathrm{d} y \leq C\|\boldsymbol{u}\|_{\mathrm{H}^{\frac{1}{2}}}\|\boldsymbol{\xi}\|_{\mathrm{H}^{\frac{1}{2}}}
$$

see e.g., [14]. We estimate $\left(\mathcal{M}_{\boldsymbol{\varphi}_{v, \mu}}(\boldsymbol{\xi}), f_{\delta_{0}} \boldsymbol{\xi}\right)_{2}$ as follows

$$
\begin{array}{r}
\left|\left(\mathcal{M}_{\boldsymbol{\varphi}_{v, \mu}}(\boldsymbol{\xi}), f_{\delta_{0}} \boldsymbol{\xi}\right)_{2}\right| \leq\left\|f_{\delta_{0}}|\boldsymbol{\xi}|^{2}\right\|_{1} \sup _{x} \int_{\mathbb{R}^{3}}\left(\frac{1}{|y|}\left(|\boldsymbol{\xi}(x+y)|^{2}+\left|\boldsymbol{\xi}(y+x) \cdot \boldsymbol{\varphi}_{v, \mu}(y+x)\right|\right)\right) \mathrm{d} y \\
+\left\|f_{\delta_{0}} \boldsymbol{\varphi}_{v, \mu} \cdot \boldsymbol{\xi}\right\|_{1} \sup _{x} \int_{\mathbb{R}^{3}} \frac{1}{|y|}|\boldsymbol{\xi}(y+x)|^{2} \mathrm{~d} y .
\end{array}
$$

Using (8.30) we find

$$
\left|\left(\mathcal{M}_{\boldsymbol{\varphi}_{v, \mu}}(\boldsymbol{\xi}), f_{\delta_{0}} \boldsymbol{\xi}\right)_{2}\right| \leq\left\|f_{\delta_{0}}|\boldsymbol{\xi}|^{2}\right\|_{1}\left(\|\boldsymbol{\xi}\|_{\mathrm{H}^{\frac{1}{2}}}^{2}+\|\boldsymbol{\xi}\|_{\mathrm{H}^{\frac{1}{2}}}\left\|\boldsymbol{\varphi}_{v, \mu}\right\|_{\mathrm{H}^{\frac{1}{2}}}\right)+\left\|f_{\delta_{0}} \boldsymbol{\varphi}_{v, \mu} \boldsymbol{\xi}\right\|_{1}\|\boldsymbol{\xi}\|_{\mathrm{H}^{\frac{1}{2}}}^{2} \text {. }
$$

Note that $\left\|f_{\delta_{0}}|\boldsymbol{\xi}|^{2}\right\|_{1}=\mathcal{Q}_{\delta_{0}}(t)$ and $\left\|f_{\delta_{0}} \boldsymbol{\varphi}_{v, \mu} \cdot \boldsymbol{\xi}\right\|_{1} \leq C\|\boldsymbol{\xi}\|_{2}$, where $C$ is independent of $\delta_{0}$. Thus

$$
\left|\left(\mathcal{M}_{\boldsymbol{\varphi}_{v, \mu}}(\boldsymbol{\xi}), f_{\delta_{0}} \boldsymbol{\xi}\right)_{2}\right| \leq c\|\boldsymbol{\xi}\|_{\mathrm{H}^{\frac{1}{2}}}^{3}+c\|\boldsymbol{\xi}\|_{\mathrm{H}^{\frac{1}{2}}}\left(1+\|\boldsymbol{\xi}\|_{\mathrm{H}^{\frac{1}{2}}}\right) \mathcal{Q}_{\delta_{0}}(t)
$$

where $c$ is independent of $\delta_{0}$.

Inserting the results in (8.28) and (8.33) into (8.19) yields

$$
\left|d_{t} \mathcal{Q}_{\delta_{0}}\right| \leq C\|\boldsymbol{\xi}\|_{\mathrm{H}^{\frac{1}{2}}}^{2}+c\|\boldsymbol{\xi}\|_{\mathrm{H}^{\frac{1}{2}}}^{3}+c\|\boldsymbol{\xi}\|_{\mathrm{H}^{\frac{1}{2}}}\left(1+\|\boldsymbol{\xi}\|_{\mathrm{H}^{\frac{1}{2}}}\right) \mathcal{Q}_{\delta_{0}}+C(\varepsilon+|\alpha|)\|\boldsymbol{\xi}\|_{2} .
$$

This concludes the proof of the proposition.

\section{Estimates of the Lyapunov functional from below}

In this section, we define a Lyapunov functional $\mathcal{S}=\mathcal{S}(t)$ as

$$
\mathcal{S}:=\mathcal{U}_{\mu, v, y}(\boldsymbol{\psi})-\mathcal{U}_{\mu, v, y}\left(\boldsymbol{\varphi}_{\zeta}\right)
$$

Here $\mathcal{U}_{\mu, v, y}$ is defined by

$$
\mathcal{U}_{\mu, v, y}(\boldsymbol{\psi}):=(\mu-V(y)) \mathcal{N}(\boldsymbol{\psi})-\frac{1}{2} v \cdot(\nabla J \boldsymbol{\psi}, \boldsymbol{\psi})_{2}+\mathcal{H}_{V}(\boldsymbol{\psi})
$$


where $\mathcal{N}(\boldsymbol{\psi}):=\frac{1}{2}\|\boldsymbol{\psi}\|_{2}^{2}$, and

$$
\mathcal{H}_{V}(\boldsymbol{\psi}):=\frac{1}{2}\left(\boldsymbol{\psi},\left(\sqrt{-\Delta+m^{2}}-m\right) \boldsymbol{\psi}\right)_{2}+\frac{1}{2}(V \boldsymbol{\psi}, \boldsymbol{\psi})_{2}-\left(\frac{1}{4|x|} *|\boldsymbol{\psi}|^{2},|\boldsymbol{\psi}|^{2}\right)_{2}
$$

The function $\mathcal{U}_{\mu, v, y}$ is a linear combination of conserved and almost conserved quantities, $\mathcal{H}_{V}, \mathcal{N}$, and $\mathcal{P}$.

The parameters $\zeta=(y, v, \vartheta, \mu)$ above are chosen such that if $\boldsymbol{\psi}$ is decomposed then $\zeta=\varsigma(\boldsymbol{\psi})$ (see Proposition 6.1, for the construction of $\varsigma$ ). In this section we show that this Lyapunov functional is coercive up to small corrections. This will be used to bound the perturbations $\boldsymbol{\xi}$ from above.

We recall the notation and a result shown in previous sections: If $\boldsymbol{\psi} \in U_{\delta}\left(\mathrm{Z}_{2}\right)$ then, under Assumption 2.1, there exists a unique decomposition of $\boldsymbol{\psi} \rightarrow(\zeta=(y, v, \vartheta, \mu), \boldsymbol{\xi})$ by $\zeta \in \mathrm{Z}_{1}$ (see Proposition 6.1).

We have the result:

Proposition 9.1. Let $\boldsymbol{\psi} \in U_{\delta}\left(\mathrm{Z}_{2}\right)$ and let Assumption 2.1 be satisfied. Denote the unique decomposition of $\boldsymbol{\psi}$ by $(\zeta, \boldsymbol{\xi})$. Let the external potential $V$ satisfy (3.1) for some number $\varepsilon>0$ and let $\rho=\rho\left(r_{1}, I_{1}\right)>0$, be defined as in Proposition B.1. Then

$$
\mathcal{S} \geq \frac{7}{8} \rho\|\boldsymbol{\xi}\|_{\mathrm{H}^{\frac{1}{2}}}^{2}-C \varepsilon \mathcal{Q}-C \varepsilon^{2}-C\|\boldsymbol{\xi}\|_{\mathrm{H}^{\frac{1}{2}}}^{4}
$$

with $\mathcal{Q}:=(\boldsymbol{\xi},|x| \boldsymbol{\xi})_{2}$.

Remark 9.2. The major limitation of $\varepsilon$ appears here. The lower bound, $\rho$, depends on the distance from zero to the start of the essential spectrum and is hence of size $\ell_{\text {sol }}^{-1}$, whereas the upper bound (next section) is given in terms of gradients of the potential, initial distances both parameterized by $\varepsilon$ together with the so far unknown size of the perturbation $\boldsymbol{\xi}$. Thus the requirement that $\varepsilon=\ell_{\text {sol }} / \ell_{\text {pot }} \ll 1$ arises here.

Proof. Using the decomposition (Proposition 6.1) of $\boldsymbol{\psi}$ into $\zeta, \boldsymbol{\xi}$, with $(y, v, \vartheta, \mu)=\zeta:=$ $\varsigma(\boldsymbol{\psi})$ we can write $\boldsymbol{\psi}$ as $U_{\delta}\left(\mathrm{Z}_{2}\right) \ni \boldsymbol{\psi}(x, \cdot)=\mathrm{e}^{-J \vartheta}\left(\boldsymbol{\varphi}_{v, \mu}(x-y)+\boldsymbol{\xi}(x-y, \cdot)\right)$. Inserting this into $\mathcal{S}$ gives

$$
\begin{aligned}
& \mathcal{S}=\mathcal{U}_{\mu, v, y}(\boldsymbol{\psi})-\mathcal{U}_{\mu, v, y}\left(\boldsymbol{\varphi}_{\zeta}\right)=\left(\mathcal{U}_{\mu, v, y}^{\prime}\left(\boldsymbol{\varphi}_{v, \mu}(\cdot-y)\right), \boldsymbol{\xi}(\cdot-y)\right)_{2} \\
&+\frac{1}{2}\left(\mathcal{U}_{\mu, v, y}^{\prime \prime}\left(\boldsymbol{\varphi}_{v, \mu}(\cdot-y)\right) \boldsymbol{\xi}(\cdot-y), \boldsymbol{\xi}(\cdot-y)\right)_{2}+\mathcal{R}_{\mu, v}\left(\boldsymbol{\xi}, \boldsymbol{\varphi}_{v, \mu}\right) \\
&=A+B+\mathcal{R}_{\mu, v}\left(\boldsymbol{\xi}, \boldsymbol{\varphi}_{v, \mu}\right) .
\end{aligned}
$$

Here we define $A$ as

$$
A:=\left(\mathcal{U}_{\mu, v, y}^{\prime}\left(\boldsymbol{\varphi}_{v, \mu}(\cdot-y)\right), \boldsymbol{\xi}(\cdot-y)\right)_{2}
$$

and $B$ as

$$
B:=\frac{1}{2}\left(\mathcal{U}_{\mu, v, y}^{\prime \prime}\left(\boldsymbol{\varphi}_{v, \mu}(\cdot-y)\right) \boldsymbol{\xi}(\cdot-y), \boldsymbol{\xi}(\cdot-y)\right)_{2}
$$


The remainder, $\mathcal{R}_{\mu, v}\left(\boldsymbol{\xi}, \boldsymbol{\varphi}_{v, \mu}\right)$ is defined as

$$
\begin{aligned}
\mathcal{R}_{\mu, v}\left(\boldsymbol{\xi}, \boldsymbol{\varphi}_{v, \mu}\right):= & \frac{1}{4}\left(\frac{1}{|x|} *\left(\left|\boldsymbol{\varphi}_{v, \mu}+\boldsymbol{\xi}\right|^{2}\right),\left|\boldsymbol{\varphi}_{v, \mu}+\boldsymbol{\xi}\right|^{2}\right)_{2}-\frac{1}{4}\left(\frac{1}{|x|} *\left(\left|\boldsymbol{\varphi}_{v, \mu}\right|^{2}\right),\left|\boldsymbol{\varphi}_{v, \mu}\right|^{2}\right)_{2} \\
& -\left(\frac{1}{|x|} *\left(\left|\boldsymbol{\varphi}_{v, \mu}\right|^{2}\right), \boldsymbol{\varphi}_{v, \mu} \cdot \boldsymbol{\xi}\right)_{2}-\frac{1}{2}\left(\frac{1}{|x|} *\left(\left|\boldsymbol{\varphi}_{v, \mu}\right|^{2}\right),|\boldsymbol{\xi}|^{2}\right)_{2} \\
& -\left(\frac{1}{|x|} *\left(\boldsymbol{\varphi}_{v, \mu} \cdot \boldsymbol{\xi}\right), \boldsymbol{\varphi}_{v, \mu} \cdot \boldsymbol{\xi}\right)_{2}
\end{aligned}
$$

$\mathcal{R}_{\mu, v}$ can also be defined directly from (9.5) as the remainder of the their given Taylor expansion of $\mathcal{U}_{\mu, v, y}(\boldsymbol{\psi})$ around $\boldsymbol{\varphi}_{v, \mu}$ to second order. Thus the rest term contains only the Taylor expansion of the nonlinear term in $\mathcal{U}_{\mu, v, y}$ which is what is written out above in detail. By expansion of the polynomials, $\mathcal{R}_{\mu, v}$ simplifies to

$$
\mathcal{R}_{\mu, v}\left(\boldsymbol{\xi}, \boldsymbol{\varphi}_{v, \mu}\right)=-\left(\frac{1}{|x|} *|\boldsymbol{\xi}|^{2}, \boldsymbol{\varphi}_{v, \mu} \cdot \boldsymbol{\xi}\right)_{2}-\frac{1}{4}\left(\frac{1}{|x|} *|\boldsymbol{\xi}|^{2},|\boldsymbol{\xi}|^{2}\right)_{2} .
$$

We now proceed to estimate the terms $A, B$ and $\mathcal{R}_{\mu, v}$. We begin with $\mathcal{R}_{\mu, v}$. The Hardy-Littlewood-Sobolev inequality yields

$$
\left|\mathcal{R}_{\mu, v}\left(\boldsymbol{\xi}, \boldsymbol{\varphi}_{v, \mu}\right)\right| \leq c\left(\|\boldsymbol{\xi}\|_{12 / 5}^{4}+\|\boldsymbol{\xi}\|_{12 / 5}^{3}\right) .
$$

From the Sobolev inequality we have $\|f\|_{12 / 5} \leq C\|f\|_{\mathrm{H}^{1 / 4}} \leq C\|f\|_{\mathrm{H}^{\frac{1}{2}}}$. Hence,

$$
\left|\mathcal{R}_{\mu, v}\left(\boldsymbol{\xi}, \boldsymbol{\varphi}_{v, \mu}\right)\right| \leq c^{\prime}\left(\|\boldsymbol{\xi}\|_{\mathrm{H}^{\frac{1}{2}}}^{4}+\|\boldsymbol{\xi}\|_{\mathrm{H}^{\frac{1}{2}}}^{3}\right) .
$$

Cauchy's inequality in the form $2 a b \leq \eta a^{2}+\eta^{-1} b^{2}$ finally yields that

$$
\left|\mathcal{R}_{\mu, v}\left(\boldsymbol{\xi}, \boldsymbol{\varphi}_{v, \mu}\right)\right| \leq C\|\boldsymbol{\xi}\|_{\mathrm{H}^{\frac{1}{2}}}^{4}+\frac{\rho}{16}\|\boldsymbol{\xi}\|_{\mathrm{H}^{\frac{1}{2}}}^{2}
$$

To estimate $A$ as defined in (9.6), we relate it to $\mathcal{E}_{v, \mu}^{\prime}$. The functional $\mathcal{E}_{v, \mu}$ is defined in $(2.17)$ as

$$
\mathcal{E}_{v, \mu}(\boldsymbol{\psi}):=\mu \mathcal{N}(\boldsymbol{\psi})-\frac{1}{2} v \cdot(\nabla J \boldsymbol{\psi}, \boldsymbol{\psi})_{2}+\mathcal{H}_{V=0}(\boldsymbol{\psi})
$$

All groundstates $\boldsymbol{\varphi}_{v, \mu}$ satisfy (2.19), that is

$$
\mathcal{E}_{v, \mu}^{\prime}\left(\boldsymbol{\varphi}_{v, \mu}\right)=0
$$

We write out the terms in $A$ explicitly and identify $\mathcal{E}_{v, \mu}^{\prime}\left(\boldsymbol{\varphi}_{v, \mu}\right)$. This gives

$$
\begin{aligned}
& A=\left(\mathcal{U}_{\mu, v, y}^{\prime}\left(\boldsymbol{\varphi}_{\zeta}(\cdot-y)\right), \boldsymbol{\xi}(\cdot-y)\right)_{2}=(\mu-V(y))\left(\boldsymbol{\varphi}_{v, \mu}, \boldsymbol{\xi}\right)_{2}-v \cdot\left(\nabla J \boldsymbol{\varphi}_{v, \mu}, \boldsymbol{\xi}\right)_{2} \\
& +\left(\mathcal{H}_{V=0}^{\prime}\left(\boldsymbol{\varphi}_{v, \mu}\right), \boldsymbol{\xi}\right)_{2}+\left(V_{y} \boldsymbol{\varphi}_{v, \mu}, \boldsymbol{\xi}\right)_{2}=\left(\mathcal{E}_{v, \mu}^{\prime}\left(\boldsymbol{\varphi}_{v, \mu}\right), \boldsymbol{\xi}\right)_{2}+\left(\left(V_{y}-V(y)\right) \boldsymbol{\varphi}_{v, \mu}, \boldsymbol{\xi}\right)_{2}
\end{aligned}
$$

Using that $\mathcal{E}_{v, \mu}^{\prime}\left(\boldsymbol{\varphi}_{v, \mu}\right)=0$ and $\left(\boldsymbol{\varphi}_{v, \mu}, \boldsymbol{\xi}\right)_{2}=0$, together with that $V$ satisfies (3.1) for some small $\varepsilon$, we find that

$$
|A|=\left|\left(\left(V_{y}-V(y)\right) \boldsymbol{\varphi}_{v, \mu}, \boldsymbol{\xi}\right)_{2}\right| \leq c_{1} \varepsilon\|\boldsymbol{\xi}\|_{2}
$$


Next we estimate $B$ defined in (9.7). We also rewrite $B$ in terms of $L_{v, \mu}:=\mathcal{E}_{v, \mu}^{\prime \prime}\left(\boldsymbol{\varphi}_{v, \mu}\right)$. A calculation, similar to the one above, shows the relation

$$
\left.(\boldsymbol{\xi}(\cdot-y)), \mathcal{U}_{\mu, v, y}^{\prime \prime}\left(\boldsymbol{\varphi}_{v, \mu}(\cdot-y)\right) \boldsymbol{\xi}(\cdot-y)\right)_{2}=\left(\boldsymbol{\xi}, L_{v, \mu} \boldsymbol{\xi}\right)_{2}+\left(\boldsymbol{\xi},\left(V_{y}-V(y)\right) \boldsymbol{\xi}\right)_{2}
$$

Denote

$$
B_{1}:=\left(\boldsymbol{\xi},\left(V_{y}-V(y)\right) \boldsymbol{\xi}\right)_{2} .
$$

On the space where $w$ is symplectically orthogonal to $\mathrm{T}_{\boldsymbol{\varphi}_{v, \mu}} \mathrm{M}$, we have

$$
\left(\boldsymbol{\xi}, L_{v, \mu} \boldsymbol{\xi}\right)_{2} \geq \rho\|\boldsymbol{\xi}\|_{\mathrm{H}^{\frac{1}{2}}}^{2}
$$

which is shown in Appendix B. Note that $\rho$ here and in Proposition B.1 depends only on $r_{1}$ and $I_{1}$.

To bound $B_{1}$, we expand $V_{y}$ around $y$ to obtain

$$
\left|B_{1}\right| \leq C \varepsilon\left\||x|^{1 / 2} \boldsymbol{\xi}\right\|_{2}^{2}
$$

Hence $B$ obeys the lower bound

$$
B \geq \rho\|\boldsymbol{\xi}\|_{\mathrm{H}^{\frac{1}{2}}}^{2}-C \varepsilon\left\||x|^{1 / 2} \boldsymbol{\xi}\right\|_{2}^{2}
$$

To complete the proof, we use estimates (9.12), (9.16) and (9.21) to obtain

$$
\mathcal{S} \geq \rho\|\boldsymbol{\xi}\|_{\mathrm{H}^{\frac{1}{2}}}^{2}-\left.C \varepsilon\|\| x\right|^{1 / 2} \boldsymbol{\xi}\left\|_{2}^{2}-c_{1} \varepsilon\right\| \boldsymbol{\xi}\left\|_{2}-C\right\| \boldsymbol{\xi}\left\|_{\mathrm{H}^{\frac{1}{2}}}^{4}-\frac{\rho}{16}\right\| \boldsymbol{\xi} \|_{\mathrm{H}^{\frac{1}{2}}}^{2}
$$

By using $c_{1} \varepsilon\|\boldsymbol{\xi}\|_{2} \leq 4 c_{1}^{2} \varepsilon^{2} / \rho+(\rho / 16)\|\boldsymbol{\xi}\|_{2}^{2}$ in the above equation we conclude the proof.

\section{Estimates of the Lyapunov functional from above}

In this section we show that the above defined Lyapunov functional $\mathcal{S}$ is almost conserved, to cubic order in terms of small quantities. First, we recall that, mass, energy are conserved and that the momentum satisfies the Ehrenfest identity i.e.,

$$
d_{t} \mathcal{N}=0, d_{t}(J \nabla \boldsymbol{\psi}, \boldsymbol{\psi})_{2}=-(\boldsymbol{\psi},(\nabla V) \boldsymbol{\psi})_{2}, d_{t} \mathcal{H}_{V}=0
$$

The conservation laws are proved in [20] and for Ehrenfest's lemma see the comment after (2.16). Once again, recall that the Lyapunov-Schmidt functional is defined as

$$
\mathcal{S}:=\mathcal{U}_{\mu, v, y}(\boldsymbol{\psi})-\mathcal{U}_{\mu, v, y}\left(\boldsymbol{\varphi}_{\zeta}\right)
$$

with $\zeta=(y, v, \vartheta, \mu)=\varsigma(\boldsymbol{\psi})$, provided that $\varsigma$ exists (see Proposition 6.1), and where

$$
\mathcal{U}_{\mu, v, y}(\boldsymbol{\psi}):=(\mu-V(y)) \mathcal{N}(\boldsymbol{\psi})-\frac{1}{2} v \cdot(\nabla J \boldsymbol{\psi}, \boldsymbol{\psi})_{2}+\mathcal{H}_{V}(\boldsymbol{\psi})
$$

We can now state the following result. 
Proposition 10.1. Let Assumption 2.1 be satisfied and let $\boldsymbol{\psi}$ be a solution to (2.15), with initial condition $\boldsymbol{\psi}_{0} \in U_{\varepsilon}\left(\mathrm{Z}_{3}\right), \varepsilon \leq \delta$. The decomposition of $\boldsymbol{\psi}$, which exists for some times, is denoted by $(\zeta=(y, v, \vartheta, \mu), \boldsymbol{\xi})$. Let $\alpha:=(\dot{y}-v, \dot{v}, \mu-\dot{\vartheta}-V(y), \dot{\mu})$, see eqn. (7.4), and let the external potential $V$ satisfy (3.1) for some small parameter $\varepsilon>0$. Finally, let $\mathcal{S}(t)$ be defined as above. Then

$$
|\mathcal{S}(t)| \leq|\mathcal{S}(0)|+C t \sup _{s \leq t}\left((\varepsilon+|\alpha(s)|)\left(\varepsilon^{2}+\|\boldsymbol{\xi}(\cdot, s)\|_{\mathrm{H}^{\frac{1}{2}}}^{2}\right)+\|\boldsymbol{\xi}(\cdot, s)\|_{\mathrm{H}^{\frac{1}{2}}}^{3}\left(1+\|\boldsymbol{\xi}(\cdot, s)\|_{\mathrm{H}^{\frac{1}{2}}}^{2}\right)\right),
$$

where $C$ depends only on $r_{j}$, and $I_{j}, j=1,2,3$.

Using Proposition 6.1, we have the following corollary.

Corollary 10.2. In addition to the assumptions in Proposition 10.1, we assume that $\left\|\boldsymbol{\psi}_{0}-\boldsymbol{\varphi}_{\zeta_{0}}\right\|_{\tilde{\Gamma}} \leq \varepsilon<\delta$, for $\varepsilon$ sufficiently small. Then

$$
|\mathcal{S}(t)| \leq C \varepsilon^{2}+C t \sup _{s \leq t}\left((\varepsilon+|\alpha(s)|)\left(\varepsilon^{2}+\|\boldsymbol{\xi}(\cdot, s)\|_{\mathrm{H}^{\frac{1}{2}}}^{2}\right)+\|\boldsymbol{\xi}(\cdot, s)\|_{\mathrm{H}^{\frac{1}{2}}}^{3}\left(1+\|\boldsymbol{\xi}(\cdot, s)\|_{\mathrm{H}^{\frac{1}{2}}}^{2}\right)\right),
$$

Proof. Expanding $\mathcal{S}(0)=\mathcal{U}_{\mu, v, y}(\boldsymbol{\psi})-\left.\mathcal{U}_{\mu, v, y}\left(\boldsymbol{\varphi}_{\zeta}\right)\right|_{t=0}$, around the soliton yields

$$
\begin{aligned}
|\mathcal{S}(0)| \leq\left|\left(\left(V_{y}-V(y)\right) \boldsymbol{\varphi}_{v, \mu}, \boldsymbol{\xi}\right)_{2}\right|+\left|\left(L_{v_{0}, \mu_{0}} \boldsymbol{\xi}, \boldsymbol{\xi}\right)_{2}\right|+\left|\left(\left(V_{y}-V(y)\right) \boldsymbol{\xi}, \boldsymbol{\xi}\right)_{2}\right| \\
+\left.\left|\mathcal{R}_{\mu, v}\left(\boldsymbol{\xi}, \boldsymbol{\varphi}_{v_{0}, \mu_{0}}\right)\right|\right|_{t=0}
\end{aligned}
$$

Here $\mathcal{R}_{\mu, v}$ is defined in (9.9). Using estimate (9.12) yields

$$
|\mathcal{S}(0)| \leq\left. C\left(\varepsilon^{2}+(1+\varepsilon)\|\boldsymbol{\xi}\|_{\mathrm{H}^{\frac{1}{2}}}^{2}+\|\boldsymbol{\xi}\|_{\mathrm{H}^{\frac{1}{2}}}^{4}\right)\right|_{t=0}
$$

To estimate $\left.\|\boldsymbol{\xi}\|_{\mathrm{H}^{\frac{1}{2}}}\right|_{t=0}$, we recall from Proposition 6.1 (ii) with $\boldsymbol{\psi}_{0} \in U_{\varepsilon}\left(\mathrm{Z}_{3}\right)$ that

$$
\left.\|\boldsymbol{\xi}\|_{\mathrm{H}^{\frac{1}{2}}}\right|_{t=0}=\left\|\boldsymbol{\psi}_{0}-\boldsymbol{\varphi}_{\varsigma\left(\boldsymbol{\psi}_{0}\right)}\right\|_{\mathrm{H}^{\frac{1}{2}}} \leq\left\|\boldsymbol{\psi}_{0}-\boldsymbol{\varphi}_{\zeta_{0}}\right\|_{\mathrm{H}^{\frac{1}{2}}}+\left\|\boldsymbol{\varphi}_{\zeta_{0}}-\boldsymbol{\varphi}_{\varsigma\left(\boldsymbol{\psi}_{0}\right)}\right\|_{\mathrm{H}^{\frac{1}{2}}} \leq C \varepsilon
$$

Here $C$ depends only on $I_{j}$ and $r_{j}, j=1,2,3$. Since $\varepsilon^{4} \leq C \varepsilon^{2}$, we find that

$$
|\mathcal{S}(0)| \leq C \varepsilon^{2}
$$

whenever $\varepsilon$ is sufficiently small.

Proof of Proposition 10.1. The proof of this proposition is a straightforward calculation. Using that

$$
\mathcal{S}(t)-\mathcal{S}(0)=\int_{0}^{t} d_{s} \mathcal{S}(s) \mathrm{d} s
$$

we find

$$
|\mathcal{S}(t)| \leq|\mathcal{S}(0)|+t \sup _{s \leq t}\left|d_{s} \mathcal{S}(s)\right|
$$

Thus the desired result corresponds to controlling $\left|d_{t} \mathcal{S}\right|$ in terms which are of third order or higher in $\varepsilon,|\alpha|$ and $\|\boldsymbol{\xi}\|_{\mathrm{H}^{\frac{1}{2}}}$. 
By Proposition 7.5 and $\boldsymbol{\psi}_{0} \in U_{\delta}\left(\mathrm{Z}_{3}\right)$ there is some positive time such that the solution $\boldsymbol{\psi}$ to (2.15) satisfies $\boldsymbol{\psi} \in U_{\delta}\left(\mathrm{Z}_{2}\right)$ to (2.15) and the it has a unique decomposition into $\zeta, \boldsymbol{\xi}$.

We now calculate the time derivative of $\mathcal{U}_{\mu, v, y}(\boldsymbol{\psi})$ for a solution $\boldsymbol{\psi}$ to (2.15). By (10.1), we find

$$
d_{t} \mathcal{U}_{\mu, v, y}(\boldsymbol{\psi})=\mathcal{N}(\boldsymbol{\psi}) d_{t}(\mu-V(y))-\frac{1}{2} \dot{v} \cdot(J \nabla \boldsymbol{\psi}, \boldsymbol{\psi})_{2}+\frac{1}{2} v \cdot(\boldsymbol{\psi}, \nabla V \boldsymbol{\psi})_{2} .
$$

Here $\mu, v$ and $y$ are taken from the decomposition of $\boldsymbol{\psi}$.

Next, we rewrite it in terms of $\mathcal{E}_{v, \mu}$ functional for the (boosted) solitary waves

$$
\begin{aligned}
\mathcal{U}_{\mu, v, y}\left(\boldsymbol{\varphi}_{v, \mu}(\cdot-y)\right) & =\mu \mathcal{N}\left(\boldsymbol{\varphi}_{v, \mu}\right)-\frac{1}{2} v \cdot\left(J \nabla \boldsymbol{\varphi}_{v, \mu}, \boldsymbol{\varphi}_{v, \mu}\right)_{2}+\mathcal{H}_{V=0}\left(\boldsymbol{\varphi}_{v, \mu}\right) \\
& +\left(\boldsymbol{\varphi}_{v, \mu}\left(V_{y}-V(y)\right), \boldsymbol{\varphi}_{v, \mu}\right)_{2}=\mathcal{E}_{v, \mu}\left(\boldsymbol{\varphi}_{v, \mu}\right)+\left(R_{V} \boldsymbol{\varphi}_{v, \mu}, \boldsymbol{\varphi}_{v, \mu}\right)_{2}
\end{aligned}
$$

where we used that the symmetry properties of $\boldsymbol{\varphi}_{v, \mu}$ to conclude that $\left|\boldsymbol{\varphi}_{v, \mu}\right|^{2}$ is even in all directions and hence $\left(\boldsymbol{\varphi}_{v, \mu} x_{j}, \boldsymbol{\varphi}_{v, \mu}\right)_{2}=0$, for $j=1,2,3$. Thus

$$
d_{t} \mathcal{U}_{\mu, v, y}\left(\boldsymbol{\varphi}_{v, \mu}(\cdot-y)\right)=\dot{\mu} \mathcal{N}\left(\varphi_{v, \mu}\right)-\frac{1}{2} \dot{v} \cdot\left(J \nabla \boldsymbol{\varphi}_{v, \mu}, \boldsymbol{\varphi}_{v, \mu}\right)_{2}+d_{t}\left(R_{V} \boldsymbol{\varphi}_{v, \mu}, \boldsymbol{\varphi}_{v, \mu}\right)_{2},
$$

where we used that $\mathcal{E}_{v, \mu}^{\prime}\left(\boldsymbol{\varphi}_{v, \mu}\right)=0$.

Subtracting (10.14) from (10.12), using the orthogonality relations $\left(\boldsymbol{\varphi}_{v, \mu}, \boldsymbol{\xi}\right)_{2}=0$ and $\left(J \nabla \boldsymbol{\varphi}_{v, \mu}, \boldsymbol{\xi}\right)_{2}=0$ gives

$$
\begin{array}{r}
d_{t} \mathcal{S}=\frac{1}{2}\|\boldsymbol{\xi}\|_{2}^{2} \dot{\mu}-\frac{1}{2} \dot{v} \cdot(J \nabla \boldsymbol{\xi}, \boldsymbol{\xi})_{2}+\frac{1}{2} v \cdot(\boldsymbol{\psi}, \nabla V \boldsymbol{\psi})_{2}-\dot{y} \cdot \nabla V(y) \mathcal{N}(\boldsymbol{\psi}) \\
-d_{t}\left(R_{V} \boldsymbol{\varphi}_{v, \mu}, \boldsymbol{\varphi}_{v, \mu}\right)_{2}
\end{array}
$$

The first two terms are of cubical order, quadratic in $\|\boldsymbol{\xi}\|_{\mathrm{H}^{\frac{1}{2}}}$ and linear in $\alpha$; recall the definition of $\alpha$ in (7.4). The last term is also of third order or higher. Indeed, let

$$
A_{2}:=d_{t}\left(R_{V} \boldsymbol{\varphi}_{v, \mu}, \boldsymbol{\varphi}_{v, \mu}\right)_{2} .
$$

Then

$$
\left|A_{2}\right|=\left|\dot{y} \cdot\left(\boldsymbol{\varphi}_{v, \mu} \nabla_{y} R_{V}, \boldsymbol{\varphi}_{v, \mu}\right)_{2}+\left(R_{V} \boldsymbol{\varphi}_{v, \mu}, \dot{\mu} \partial_{\mu} \boldsymbol{\varphi}_{v, \mu}+\dot{v} \cdot \nabla_{v} \boldsymbol{\varphi}_{v, \mu}\right)_{2}\right| .
$$

To bound $A_{2}$, we recall that $|v| \leq 1$ and that $\dot{y}_{j}=v_{j}-Y_{j}(\zeta, \boldsymbol{\xi}), j=1,2,3$ (see (7.9)) together with equation (7.5). This gives the estimate for $Y_{j}$; see Proposition 7.1. We thus find

$$
\left.\left|A_{2}\right| \leq C \varepsilon^{2}(|\alpha|+\varepsilon|Y|)\right) .
$$

The middle two terms of (10.15) are also of at least cubic order, Indeed, let

$$
A_{1}:=\frac{1}{2} v \cdot(\boldsymbol{\psi}, \nabla V \boldsymbol{\psi})_{2}-\dot{y} \cdot \nabla V(y) \mathcal{N}(\boldsymbol{\psi}) .
$$


Decomposing $\boldsymbol{\psi}$ gives

$$
\begin{aligned}
A_{1}=\frac{1}{2} v \cdot\left(\left(\boldsymbol{\varphi}_{v, \mu}, \nabla V_{y} \boldsymbol{\varphi}_{v, \mu}\right)_{2}+2\left(\boldsymbol{\xi}, \nabla V_{y} \boldsymbol{\varphi}_{v, \mu}\right)_{2}\right. & \left.+\left(\boldsymbol{\xi}, \nabla V_{y} \boldsymbol{\xi}\right)_{2}\right) \\
& -\frac{1}{2} \dot{y} \cdot \nabla V(y)\left(\|\boldsymbol{\xi}\|_{2}^{2}+\left\|\boldsymbol{\varphi}_{v, \mu}\right\|_{2}^{2}\right)
\end{aligned}
$$

Using the orthogonality relation $\left(\boldsymbol{\xi}, \boldsymbol{\varphi}_{v, \mu}\right)_{2}=0$, and $\left(\boldsymbol{\varphi}_{v, \mu}, x_{j} \boldsymbol{\varphi}_{v, \mu}\right)_{2}=0$, we find

$$
\begin{aligned}
A_{1}=\frac{1}{2} v & \left(\left(\boldsymbol{\varphi}_{v, \mu}, \nabla_{y} R_{V} \boldsymbol{\varphi}_{v, \mu}\right)_{2}+\left(\boldsymbol{\xi},\left(\nabla V_{y}-\nabla V(y)\right) \boldsymbol{\xi}\right)_{2}\right) \\
& +\frac{1}{2}(v-\dot{y}) \cdot \nabla V(y)\left(\|\boldsymbol{\xi}\|_{2}^{2}+\left\|\boldsymbol{\varphi}_{v, \mu}\right\|_{2}^{2}\right)+v \cdot\left(\boldsymbol{\xi},\left(\nabla V_{y}-\nabla V(y)\right) \boldsymbol{\varphi}_{v, \mu}\right)_{2}
\end{aligned}
$$

Hence,

$$
\left|A_{1}\right| \leq C \varepsilon\left(\varepsilon^{2}+\|\boldsymbol{\xi}\|_{2}^{2}+|Y|\left(1+\|\boldsymbol{\xi}\|_{\mathrm{H}^{\frac{1}{2}}}^{2}\right)\right),
$$

with $Y$ defined as in Proposition 7.1. From this, we infer

$$
\left|d_{t} \mathcal{S}\right| \leq C|\alpha|\|\boldsymbol{\xi}\|_{\mathrm{H}^{\frac{1}{2}}}^{2}+\left|A_{1}\right|+\left|A_{2}\right|
$$

Inserting the above estimates for $A_{1}$ and $A_{2}$ gives

$$
\left|d_{t} \mathcal{S}\right| \leq C(\varepsilon+|\alpha|)\left(\|\boldsymbol{\xi}\|_{\mathrm{H}^{\frac{1}{2}}}^{2}+\varepsilon^{2}\right)+C \varepsilon|Y|\left(1+\varepsilon^{2}+\|\boldsymbol{\xi}\|_{\mathrm{H}^{\frac{1}{2}}}^{2}\right)
$$

Note that $\varepsilon \leq C$, inserting the bound of $|Y|$, given in (7.5), we simplify the above result to obtain

$$
\left|d_{t} \mathcal{S}\right| \leq C(\varepsilon+|\alpha|)\left(\|\boldsymbol{\xi}\|_{\mathrm{H}^{\frac{1}{2}}}^{2}+\varepsilon^{2}\right)+C\|\boldsymbol{\xi}\|_{\mathrm{H}^{\frac{1}{2}}}^{3}\left(1+\|\boldsymbol{\xi}\|_{\mathrm{H}^{\frac{1}{2}}}^{2}\right)
$$

For some constant $0<C<\infty$ depending only on $r_{j}$ and $I_{j}, j=1,2,3$.

\section{Proof of Theorem 3.1}

In this section we use the lower and upper bound on the Lyapunov functional together with the modulation equations to bound $\|\boldsymbol{\xi}\|_{\tilde{\Gamma}}$ and $|\alpha|$.

Proof of Theorem 3.1. The Theorem 3.1 assumes that Assumption 2.1 is satisfied and that the external potential satisfies (3.1) for some $\varepsilon>0$. Furthermore, we require that the initial condition $\boldsymbol{\psi}_{0}$ satisfies the inequality

$$
\left\|\psi_{0}-\varphi_{\zeta_{0}}\right\|_{\tilde{\Gamma}} \leq \varepsilon
$$

for some $\zeta_{0} \in \mathrm{Z}_{3}$. By Proposition $7.5 \boldsymbol{\psi}(\cdot, t) \in U_{\delta}\left(\mathrm{Z}_{2}\right)$, for some $\delta$ and up to some time $T_{\delta}$. Here $U_{\delta}$ is constructed in Proposition 6.1. Thus all the assumptions for Corollary 10.2 are satisfied, and we obtain

$$
|\mathcal{S}(t)| \leq C \varepsilon^{2}+C t \sup _{s \leq t} f(s),
$$


where

$$
f(s)=(\varepsilon+|\alpha(s)|)\left(\varepsilon^{2}+\|\boldsymbol{\xi}(\cdot, s)\|_{\mathrm{H}^{\frac{1}{2}}}^{2}\right)+\|\boldsymbol{\xi}(\cdot, s)\|_{\mathrm{H}^{\frac{1}{2}}}^{3}\left(1+\|\boldsymbol{\xi}(\cdot, s)\|_{\mathrm{H}^{\frac{1}{2}}}^{2}\right) .
$$

For times $t \leq T_{\delta}$, we can invoke Proposition 9.1 and Corollary 10.2 to find

$$
\frac{7}{8} \rho\|\boldsymbol{\xi}(\cdot, t)\|_{\mathrm{H}^{\frac{1}{2}}}^{2} \leq C \varepsilon^{2}+C \sup _{s \leq t}\left(t f(s)+\varepsilon\left\||x|^{1 / 2} \boldsymbol{\xi}(\cdot, s)\right\|_{2}^{2}+\|\boldsymbol{\xi}(\cdot, s)\|_{\mathrm{H}^{\frac{1}{2}}}^{4}\right) .
$$

Thus, for all such times $t \leq T_{\delta}$ we have the above inequality, and since the right-hand side is independent under $t \rightarrow s^{\prime}, \sup _{s^{\prime} \leq t}$, for $t \leq T_{\delta}$ we can also apply this to the left hand side. This gives us

$$
\frac{7}{8} \rho \sup _{s \leq t}\|\boldsymbol{\xi}(\cdot, s)\|_{\mathrm{H}^{\frac{1}{2}}}^{2} \leq C \varepsilon^{2}+C \sup _{s \leq t}\left(t f(s)+\varepsilon\left\||x|^{1 / 2} \boldsymbol{\xi}(\cdot, s)\right\|_{2}^{2}+\|\boldsymbol{\xi}(\cdot, s)\|_{\mathrm{H}^{\frac{1}{2}}}^{4}\right) .
$$

Consider the inequality

$$
t \leq \frac{\rho}{8 C} \frac{1}{\varepsilon+\sup _{s \leq t}\left(|\alpha(s)|+\|\boldsymbol{\xi}(\cdot, s)\|_{\mathrm{H}^{\frac{1}{2}}}\right)}
$$

This inequality implicitly defines a maximal time, $T_{2}$, dependent on $\varepsilon$, the size of $|\alpha|$ and $\|\boldsymbol{\xi}\|_{\mathrm{H}^{\frac{1}{2}}}$, such that when $t \leq T_{2}$ the inequality holds. We now choose the minimal time of $T_{2}$ and $T_{\delta}$. Since this minimal time is necessarily smaller than the right-hand side of the inequality (11.6), we can use this inequality to re-write (11.5) as

$$
\begin{aligned}
\frac{7}{8} \rho \sup _{s \leq t}\|\boldsymbol{\xi}(\cdot, s)\|_{\mathrm{H}^{\frac{1}{2}}}^{2} \leq C \varepsilon^{2}+\sup _{s \leq t}\left(\frac{\rho}{8}\left(\varepsilon^{2}+2\|\boldsymbol{\xi}(\cdot, s)\|_{\mathrm{H}^{\frac{1}{2}}}^{2}+\|\boldsymbol{\xi}(\cdot, s)\|_{\mathrm{H}^{\frac{1}{2}}}^{4}\right)\right. \\
\left.+C\left(\varepsilon\left\||x|^{1 / 2} \boldsymbol{\xi}(\cdot, s)\right\|_{2}^{2}+\|\boldsymbol{\xi}(\cdot, s)\|_{\mathrm{H}^{\frac{1}{2}}}^{4}\right)\right) .
\end{aligned}
$$

Let $T_{1}$ be the maximal time such that for $t \leq T_{1}$ Eqn. (8.3) in Corollary 8.2 holds. By choosing the minimal of the three times $T_{\delta}, T_{1}$ and $T_{2}$ we can apply the result in Corollary 8.2. That is, we use

$$
\sup _{s \leq t}\left\||x|^{1 / 2} \boldsymbol{\xi}(\cdot, s)\right\|_{2}^{2} \leq C \varepsilon+\sup _{s \leq t}\|\boldsymbol{\xi}(\cdot, s)\|_{\mathrm{H}^{\frac{1}{2}}}
$$

in (11.7) above. We find, for this minimal time,

$$
\frac{1}{2} \rho \sup _{s \leq t}\|\boldsymbol{\xi}(\cdot, s)\|_{\mathrm{H}^{\frac{1}{2}}}^{2} \leq C\left(\varepsilon^{2}+\sup _{s \leq t}\|\boldsymbol{\xi}(\cdot, s)\|_{\mathrm{H}^{\frac{1}{2}}}^{4}\right) .
$$

Recalling that the initial condition is small enough we simplify the inequality to find

$$
\|\boldsymbol{\xi}(\cdot, t)\|_{\mathrm{H}^{\frac{1}{2}}}^{2} \leq \sup _{s \leq t}\|\boldsymbol{\xi}(\cdot, s)\|_{\mathrm{H}^{\frac{1}{2}}}^{2} \leq C \varepsilon^{2}
$$


We now use the definition (2.11) to find from (11.10) and (11.8) that

$$
\|\boldsymbol{\xi}\|_{\tilde{\Gamma}} \leq C \varepsilon
$$

We insert the result (11.10) into the modulation equations, (7.3), we find that $|\alpha|$, as defined in Proposition 7.1, satisfies the inequality

$$
\sup _{t \leq s}|\alpha(s)| \leq C^{\prime} \sup _{s \leq t}(|\alpha(s)| \varepsilon+2 \varepsilon)
$$

Choosing $\varepsilon$ sufficiently small, i.e., $C^{\prime} \varepsilon \leq 1 / 2$, leads to

$$
\sup _{t \leq s}|\alpha(s)| \leq c^{\prime} \varepsilon .
$$

This inserted into (7.5) gives that $\left|Y_{j}\right| \leq C \varepsilon^{2}$, for all $j$ and hence the finite dimensional modulation equations are bounded by $C \varepsilon^{2}$. We insert the above upper bounds on $\|\boldsymbol{\xi}\|_{2}$, $|\alpha|$, into the inequalities (8.3) and (11.6) that determines the times $T_{1}$ and $T_{2}$, both inequalities simplify to

$$
t \leq \frac{c}{\varepsilon} .
$$

By possibly reducing the constant $c$ we find that $c / \varepsilon<T_{\delta}$, and we have proved the theorem.

\section{Acknowledgements}

Lars Jonsson and Jürg Fröhlich are grateful to the Swiss National Foundation (NFProject 20-105493).

\section{A Proof of Proposition 4.3(v)}

Here we prove Proposition 4.3(v). In [9, App. C] we showed that the essential spectrum starts at $\mu-\mu_{l}(v)$, where $\mu_{l}(v)=\left(1-\sqrt{1-v^{2}}\right) m$. For the remaining claims we have:

Proposition A.1. Suppose Assumption 2.1 is satisfied for the frequency $\mu_{0}$. There is a neighborhood, $W \subset \mathbb{R}^{2}$, around $\left(0, \mu_{0}\right)$ such that

$$
\operatorname{dim} \operatorname{Ker}\left(L_{v, \mu}\right)=4,
$$

for all $(|v|, \mu) \in W$. Furthermore, $L_{v, \mu}$ has exactly one negative eigenvalue, and around zero there is a gap to the next spectral point.

Proof. To prove the proposition, we begin with the point $(v, \mu)=\left(0, \mu_{0}\right)$, here $\boldsymbol{\varphi}_{\mu}=$ $\left(\varphi_{\mu}, 0\right)$ and $L_{v, \mu}$ reduces to $L_{\mu_{0}}=\operatorname{diag}\left(L_{11, \mu_{0}}, L_{22, \mu_{0}}\right)$. That Assumption 2.1 implies that $\operatorname{dim} \operatorname{Ker}\left(L_{\mu_{0}}\right)=4$ has already been shown in the proof of Proposition 4.5. 
For general velocities, $v \neq 0$, and frequencies, let $\mathrm{K}:=\operatorname{Ker}\left(L_{v, \mu}\right)$ and let $k=\operatorname{dim} \mathrm{K}$. Equations (4.4) show that

$$
J \boldsymbol{\varphi}_{v, \mu}, \partial_{j} \boldsymbol{\varphi}_{v, \mu} \in \mathrm{K}
$$

and consequently $k \geq 4$, to show that $k=4$, we use Kato's perturbation of the spectrum: Define the operator $A:=L_{v, \mu}-L_{\mu_{0}}=-v \cdot \nabla J+\mathcal{E}_{0, \mu}^{\prime \prime}\left(\boldsymbol{\varphi}_{v, \mu}\right)-\mathcal{E}_{0, \mu_{0}}^{\prime \prime}\left(\boldsymbol{\varphi}_{\mu_{0}}, 0\right)$, it is $L_{\mu_{0}}{ }^{-}$ bounded;

$$
\|A \boldsymbol{u}\|_{2} \leq c_{|v|, \mu-\mu_{0}}\|\boldsymbol{u}\|_{2}+C_{|v|, \mu-\mu_{0}}\left\|L_{\mu_{0}} \boldsymbol{u}\right\|_{2},
$$

where both constants approach zero as both $|v|$ and $\left|\mu-\mu_{0}\right|$ approach zero, which follows from the fact $\left\|\boldsymbol{\varphi}_{v, \mu}-\boldsymbol{\varphi}_{\mu_{0}}\right\|_{\mathrm{H}^{1}} \leq\left(|v|+\left|\mu-\mu_{0}\right|\right) C$, for small enough $|v|$ and $\left|\mu-\mu_{0}\right|$.

Denote the spectral distance in $\sigma\left(L_{\mu_{0}}\right)$ from zero to nearest spectral point $d$ and consider the inequality:

$$
c_{|v|, \mu-\mu_{0}}+C_{v, \mu-\mu_{0}} d \leq d / 2
$$

For neighborhoods $W$ such that the above inequality is satisfied for all $(|v|, \mu) \in W,[17$, $\S . V .4 .3]$ states that within the circle with center zero and radius $d / 2$ there are exactly four (repeated) eigenvalues of $L_{v, \mu}$ (since $L_{\mu_{0}}$ has a degeneracy four zero eigenvalue). Thus $k=\operatorname{dim} K \leq 4$ consequently $k=4$. Furthermore, $\sigma\left(L_{v, \mu}\right)$ has a spectral gap of at least $d / 2$ from zero to the next spectral point. The circle thus separates the spectrum into three parts.

The function $\boldsymbol{\varphi}_{\mu_{0}}$ is a minimizer with one constraint, thus its corresponding Hessian $L_{\mu_{0}}$, can have at most one negative eigenvalue, see e.g., [8]. But by

$$
\left(\partial_{\mu} \boldsymbol{\varphi}_{\mu}, L_{\mu} \partial_{\mu} \boldsymbol{\varphi}_{\mu}\right)_{2}=-\mathcal{N}^{\prime}\left(\boldsymbol{\varphi}_{\mu}\right)<0 \text {. }
$$

it has at least one negative eigenvalue. Thus it has exactly one negative eigenvalue. The above separation of the spectrum together with the fact that the eigenvalues (of self-adjoint operators) are constrained to the real axis ensure that $L_{v, \mu}$ has exactly one negative eigenvalue.

\section{B Positivity of $\left(L_{v, \mu} \varphi_{v}, \varphi_{v}\right)_{2}$}

In this section we show that $\left(\boldsymbol{\xi}, L_{v, \mu} \boldsymbol{\xi}\right)_{2} \geq \rho\|\boldsymbol{\xi}\|_{2}^{2}$ for $\omega(\boldsymbol{\xi}, \boldsymbol{z})=0$ for all $\boldsymbol{z} \in \mathrm{T}_{\boldsymbol{\varphi}_{\zeta}} \mathrm{M}_{1}$. From Proposition 4.3 we know that $L_{v, \mu}$ has one negative eigenvalue. We have the following result

Proposition B.1. Under Assumption 2.1 and with $|v|<r_{1}<1$ and $\mu \in I_{1}$ there is a $\rho>0$ dependent only on $I_{1}$ and $r_{1}$ defined in Proposition 5.1 such that if $\omega(\boldsymbol{\xi}, \boldsymbol{z})=0$ for all $\boldsymbol{z} \in \mathrm{T}_{\boldsymbol{\varphi}_{\zeta}} \mathrm{M}_{1}$, then

$$
\left(\boldsymbol{\xi}, L_{v, \mu} \boldsymbol{\xi}\right)_{2} \geq \rho\|\boldsymbol{\xi}\|_{\mathrm{H}^{\frac{1}{2}}}^{2}
$$

We follow the proof of Proposition D.1 in [8] with necessary modifications to the pseudo-relativistic Hartree equation. But we repeat the proof here for completeness. We break the proposition into three steps. 
Lemma B.2 (Step 1). Let $X_{1}:=\left\{\boldsymbol{\xi} \in \mathrm{H}^{\frac{1}{2}}:\|\boldsymbol{\xi}\|_{2}=1,\left(\boldsymbol{\xi}, \boldsymbol{\varphi}_{v, \mu}\right)_{2}=0\right\}$, and $|v| \leq r_{1}$, $\mu \in I_{1}$. Then

$$
\inf _{\boldsymbol{\xi} \in X_{1}}\left(\boldsymbol{\xi}, L_{v, \mu} \boldsymbol{\xi}\right)_{2}=0
$$

Proof. Let $a:=\inf _{\boldsymbol{\xi} \in X_{1}}\left(\boldsymbol{\xi}, L_{v, \mu} \boldsymbol{\xi}\right)_{2}$. Clearly $\nu \leq a \leq 0$, where $\nu<0$ is the negative eigenvalue of $L_{v, \mu}$. That $a \leq 0$ is clear as $\boldsymbol{\xi}=J \boldsymbol{\varphi}_{v, \mu} /\left\|\boldsymbol{\varphi}_{v, \mu}\right\|_{2} \in X_{1}$ yields $\left(\boldsymbol{\xi}, L_{v, \mu} \boldsymbol{\xi}\right)_{2}=0$. Moreover $a \neq \nu$. Indeed if $a=\nu$, then the (local) minimizer, $\phi$, of (B.2) would be an eigen-function of $L_{v, \mu}$ corresponding to the smallest eigenvalue $\nu$ and $\phi \in X_{1}$ and $\boldsymbol{\varphi}_{v, \mu} \perp \boldsymbol{\phi}$. Now, since $\boldsymbol{\varphi}_{v, \mu} \perp \operatorname{Ker}\left(L_{v, \mu}\right)$ and since $\nu$ is the only negative eigenvalue, we conclude that $\boldsymbol{\varphi}_{v, \mu}$ is in the spectral subspace of $L_{v, \mu}$ corresponding to the interval $[\delta, \infty)$ for some $\delta>0$. Therefore $L_{v, \mu}^{-1} \boldsymbol{\varphi}_{v, \mu}$ is well defined and $\left(\boldsymbol{\varphi}_{v, \mu}, L_{v, \mu}^{-1} \boldsymbol{\varphi}_{v, \mu}\right)_{2}>0$. On the other hand the equation $L_{v, \mu} \partial_{\mu} \boldsymbol{\varphi}_{v, \mu}=-\boldsymbol{\varphi}_{v, \mu}$ implies that

$$
\left(\boldsymbol{\varphi}_{v, \mu}, L_{v, \mu}^{-1} \boldsymbol{\varphi}_{v, \mu}\right)_{2}=-N^{\prime}\left(\boldsymbol{\varphi}_{v, \mu}\right)<0
$$

which contradicts $\left(\boldsymbol{\varphi}_{v, \mu}, L_{v, \mu}^{-1} \boldsymbol{\varphi}_{v, \mu}\right)_{2}>0$. Hence $a=\nu$ is impossible.

To show that $a=0$ we use the Euler-Lagrange equations corresponding to (B.2)

$$
L_{v, \mu} \boldsymbol{\xi}=a \boldsymbol{\xi}+b \boldsymbol{\varphi}_{v, \mu},
$$

where $a$ and $b$ are Lagrange multipliers corresponding to $\|\boldsymbol{\xi}\|_{2}=1$ and $\left(\boldsymbol{\varphi}_{v, \mu}, \boldsymbol{\xi}\right)_{2}=0$ respectively. Assume $\nu<a<0$. If $b=0$, then $a$ would be a negative eigenvalue in $(\nu, 0)$ which contradicts that $\nu$ is the only negative eigenvalue. Thus $b \neq 0$. Given $\nu<a<0$, we can solve the Euler-Lagrange equation as

$$
\boldsymbol{\xi}=b\left(L_{v, \mu}-a\right)^{-1} \boldsymbol{\varphi}_{v, \mu} .
$$

The inner product of the equation above with $\boldsymbol{\varphi}_{v, \mu}$, together with the orthogonality relation $\left(\boldsymbol{\varphi}_{v, \mu}, \boldsymbol{\xi}\right)_{2}=0$, and $b \neq 0$, give

$$
0=\left(\boldsymbol{\varphi}_{v, \mu},\left(L_{v, \mu}+|a|\right)^{-1} \boldsymbol{\varphi}_{v, \mu}\right)_{2}=: q(|a|)
$$

$q(\lambda)$ is analytic in $\lambda \in(0,|\nu|)$, and hence differentiable. Moreover it is monotonically decreasing, since

$$
q^{\prime}(\lambda)=-\left(\boldsymbol{\varphi}_{v, \mu},\left(L_{v, \mu}+\lambda\right)^{-2} \boldsymbol{\varphi}_{v, \mu}\right)_{2}=-\left\|\left(L_{v, \mu}+\lambda\right)^{-1} \boldsymbol{\varphi}_{v, \mu}\right\|_{2}^{2}<0 .
$$

Furthermore by (B.3) $q(0)=\left(\boldsymbol{\varphi}_{v, \mu}, L_{v, \mu}^{-1} \boldsymbol{\varphi}_{v, \mu}\right)_{2}<0$. Thus $q(|a|) \neq 0$, for $a \in(\nu, 0)$, which contradicts (B.6). Hence $a=0$

Lemma B.3 (Step 2). Let $X:=\left\{\boldsymbol{\xi} \in \mathrm{H}^{\frac{1}{2}}:\|\boldsymbol{\xi}\|_{2}=1, \omega(\boldsymbol{\xi}, z)=0, \forall z \in \mathrm{T}_{\boldsymbol{\varphi}_{\zeta}} \mathrm{M}_{1}\right\}$. Then

$$
\inf _{\boldsymbol{\xi} \in X}\left(\boldsymbol{\xi}, L_{v, \mu} \boldsymbol{\xi}\right)_{2}>0
$$


Proof. The Euler-Lagrange equation corresponding to (B.8) is

$$
L_{v, \mu} \boldsymbol{\xi}=a \boldsymbol{\xi}+\sum_{k} \gamma_{k} J \boldsymbol{z}_{k}
$$

where $\left\{\boldsymbol{z}_{k}\right\}$ is a basis for $\mathrm{T}_{\boldsymbol{\varphi}_{\zeta}} \mathrm{M}_{1}$. Here $a$ and $\left\{\gamma_{k}\right\}$ are the Lagrange multipliers corresponding to the constraints $\|\boldsymbol{\xi}\|_{2}=1$ and $\omega\left(\boldsymbol{\xi}, \boldsymbol{z}_{k}\right)=0 \forall k$ respectively. Note that $a=(\boldsymbol{\xi}, L \boldsymbol{\xi})_{2}$, and that $\mathrm{X} \subset \mathrm{X}_{1}$, hence $a \geq 0$. Assume that $a=0$, and that $\gamma_{j} \neq 0$ for some $j$. Then, by Corollary 5.2 , there exists a $\boldsymbol{z}=\sum_{j, l} \gamma_{j}\left(\Omega_{\boldsymbol{\varphi}_{v, \mu}}^{-1}\right)_{j l} \boldsymbol{z}_{l} \in \mathrm{T}_{\boldsymbol{\varphi}_{\zeta}} \mathrm{M}_{1}$ such that

$$
(\boldsymbol{z}, L \boldsymbol{\xi})_{2}=\sum_{j, k, l} \gamma_{j}\left(\Omega_{\boldsymbol{\varphi}_{v, \mu}}^{-1}\right)_{j l}\left(\Omega_{\boldsymbol{\varphi}_{v, \mu}}\right)_{l k} \gamma_{k}=\sum_{j}\left|\gamma_{j}\right|^{2}>0
$$

which contradicts $\left(\boldsymbol{z}, L_{v, \mu} \boldsymbol{\xi}\right)_{2}=\left(L_{v, \mu} \boldsymbol{z}, \boldsymbol{\xi}\right)_{2}=0$. Here we have used that $\boldsymbol{z}=\sum_{j} b_{j} \boldsymbol{z}_{j}$ and $\boldsymbol{z}_{j}$ is either a zero-eigenfunction or an associated zero-mode for $L_{v, \mu}$. Thus either $a>0$ or $a=0$ and $\gamma_{j}=0$. Consider the latter case. In this case

$$
L_{v, \mu} \boldsymbol{\xi}=0
$$

which implies that $\boldsymbol{\xi} \in \operatorname{Ker}\left(L_{v, \mu}\right)$. Since $\operatorname{Ker}\left(L_{v, \mu}\right) \subset \mathrm{T}_{\boldsymbol{\varphi}_{\zeta}} \mathrm{M}_{1}$, the relation $\omega(\boldsymbol{\xi}, \boldsymbol{z})=0$ for all $\boldsymbol{z} \in \mathrm{T}_{\boldsymbol{\varphi}_{\zeta}} \mathrm{M}_{1}$ contradicts the non-degeneracy of $\Omega_{\boldsymbol{\varphi}_{v, \mu}}$ on $\mathrm{M}_{1}$ (see Corollary 5.2). Thus $a>0$.

Step 3. End of Proof. Equation (B.8) implies that there exists a $\rho^{\prime}>0$ such that

$$
\left(\boldsymbol{\xi}, L_{v, \mu} \boldsymbol{\xi}\right)_{2} \geq \rho^{\prime}\|\boldsymbol{\xi}\|_{2}^{2}
$$

for some $\rho^{\prime}=\rho^{\prime}(\mu, v)$ and all $\boldsymbol{\xi} \in X$. To improve the coercivity from $\mathrm{L}^{2}$ to $\mathrm{H}^{\frac{1}{2}}$, we let $0<\delta<1$, and estimate $\left(\boldsymbol{\xi}, L_{v, \mu} \boldsymbol{\xi}\right)_{2}$ using (B.12) as

$$
(1-\delta) \rho^{\prime}\|\boldsymbol{\xi}\|_{2}^{2}+\delta\left(\boldsymbol{\xi}, L_{v, \mu} \boldsymbol{\xi}\right)_{2} \leq\left(\boldsymbol{\xi}, L_{v, \mu} \boldsymbol{\xi}\right)_{2}
$$

Upon using the explicit form of $L_{v, \mu}$ we find that

$$
\left(\boldsymbol{\xi}, L_{v, \mu} \boldsymbol{\xi}\right)_{2} \geq\left(\boldsymbol{\xi}, \sqrt{-\Delta+m^{2}} \boldsymbol{\xi}\right)_{2}-v \cdot(J \nabla \boldsymbol{\xi}, \boldsymbol{\xi})_{2}-C_{\mu}\|\boldsymbol{\xi}\|_{2}^{2},
$$

where

$$
C_{\mu} \leq|m-\mu|+C(3,1)\left\|\boldsymbol{\varphi}_{v, \mu}\right\|_{3}^{2}+\left.\sup _{x}\left|\frac{1}{|x|} *\right| \boldsymbol{\varphi}_{v, \mu}\right|^{2} \mid .
$$

Here we have used Kato's inequality (see (8.30)) and the Hardy-Littlewood-Sobolev inequality with sharp constant $C(3,1)$ see e.g., in [21, Thm. 4.3]. The two estimates (B.13), (B.14) with $\delta:=\rho^{\prime}\left(1+\rho^{\prime}+C_{\mu}\right)^{-1}$ imply

$$
\left(\boldsymbol{\xi}, L_{v, \mu} \boldsymbol{\xi}\right)_{2} \geq \rho\|\boldsymbol{\xi}\|_{\mathrm{H}^{\frac{1}{2}}}^{2},
$$

where $0<\rho=\inf _{\mu \in I_{1}, v<r_{1}}(1-|v|) \rho^{\prime}\left(1+\rho^{\prime}+C_{\mu}\right)^{-1}$. Thus for $\mu \in I_{1},|v|<r_{1}$, we find that $\rho$ depends only on $I_{1}$ and $r_{1}$. This concludes the proof of Proposition B.1. 


\section{Proof of Corollary 6.6}

In this appendix we prove Corollary 6.6 by using the proof of an implicit function theorem as proven in [6, Thm. 10.2.1] and [36, Thm 4E]. From these proofs we find that it suffices to consider three restrictions of the radii. They are 1) distance to the boundary 2) sufficient conditions for contraction 3) differentiability of $\varsigma$. We follow closely the proof of the implicit function theorem in [6, Thm. 10.2.1], applied to this case and with bounds expressed in terms of known quantities e.g., maps of ground states.

Let $p \in[1, \infty]$ and consider the $p$-metric on $\mathbb{R}^{8}$,

$$
\left|\zeta-\zeta^{\prime}\right|_{(p)}:=\left(\sum_{j}\left|\zeta_{j}-\zeta_{j}^{\prime}\right|^{p}\right)^{1 / p}
$$

These metrics are all equivalent, and it does not matter for the result which of these we use. But to make a definite choice we use the $p=2$ metric as the default one, and denote the distance $|\cdot|:=|\cdot|_{(2)}$. To obtain uniform bounds, we use $|\cdot|_{\infty}$ and $|\cdot|_{1, \infty}$ defined by

$$
\left.\left|\Omega_{\boldsymbol{\varphi}_{v, \mu}}\right|_{\infty}:=\sup _{\mu \in I_{2}^{\prime},|v|<r_{2}^{\prime}\left|\zeta^{\prime}\right|=1} \sup _{k} \mid \sum_{\boldsymbol{\varphi}_{v, \mu}}\right)_{\cdot k} \zeta_{k}^{\prime} \mid
$$

and

$$
\left.\left|\Omega_{\left.\boldsymbol{\varphi}_{v, \mu}\right|_{1, \infty}}:=\right| \partial_{\mu} \Omega_{\boldsymbol{\varphi}_{v, \mu}}\right|_{\infty}+\sum_{l=1}^{3}\left|\partial_{v_{l}} \Omega_{\boldsymbol{\varphi}_{v, \mu}}\right|_{\infty}
$$

To elucidate our proof we make a change of notation to emphasize the difference between the center of the specific balls $B_{\rho_{2}}^{8}$ and $b_{R_{2}}$ from any point on the soliton manifold $\mathrm{M}_{1}$ : The center of the balls are denoted by $\zeta^{(c)}=\left(y^{(c)}, v^{(c)}, \vartheta^{(c)}, \mu^{(c)}\right)$ and $\boldsymbol{\varphi}_{\zeta^{(c)}}$ respectively, whereas $\zeta$ ( or $\boldsymbol{\varphi}_{\zeta}$ ) is any point in $\mathrm{Z}_{2}^{\prime}$ ( or in $\mathrm{M}_{2}^{\prime}$ ).

The size of the radii clearly depend on how close to the boundary of the soliton manifold (parameter space) the point $\boldsymbol{\varphi}_{\zeta^{(c)}}\left(\zeta^{(c)}\right)$ is located. In order to obtain uniform radii, consider $\left(r_{2}^{\prime}, I_{2}^{\prime}\right)$ such that $0<r_{2}^{\prime}<r_{1}$ and $I_{2}^{\prime}$ a non-empty, open interval with $I_{2}^{\prime} \Subset I_{1}$. By the implicit function theorem there are balls in the domain $\mathrm{Z}_{1}, \mathrm{M}_{1}$ such that Lemma 6.4 holds. The symplectic manifold $\mathrm{M}_{1}$ is well defined up to and including its boundary and by the argument before, the only boundary directions are the $v$ and $\mu$ directions. Thus the radii have to satisfy the inequalities

$$
\begin{array}{r}
R_{2}<\inf _{\mu \in \partial I_{1},|v|=r_{1}, \mu^{(c)} \in \partial I_{2}^{\prime},\left|v^{(c)}\right|=r_{2}^{\prime}}\left\|\boldsymbol{\varphi}_{v, \mu}-\boldsymbol{\varphi}_{v^{(c)}, \mu^{(c)}}\right\|_{\tilde{\Gamma}}, \\
\rho_{2}<\min \left(\left|r_{1}-r_{2}^{\prime}\right|, \min _{\mu \in \partial I_{1}, \mu^{(c)} \in \partial I_{2}^{\prime}}\left|\mu-\mu^{(c)}\right|\right),
\end{array}
$$

which depend only on the choice of $r_{2}^{\prime}$ and $I_{2}^{\prime}$.

Now given a point on the manifold $\varphi_{\zeta^{(c)}} \in \mathrm{M}_{2}^{\prime}$, with its corresponding ball $b_{R_{1}}$. The second restriction on the radii is the contraction restriction which we consider by recasting the equation $G(\boldsymbol{\psi}, \zeta)=0$ into a contraction equation: $g(\boldsymbol{\psi}, \zeta)=\zeta$ where

$$
g_{j}(\boldsymbol{\psi}, \zeta):=\zeta_{j}+\sum_{k}\left(\left(\Omega^{(c)}\right)^{-1}\right)_{j k} G_{k}(\boldsymbol{\psi}, \zeta)
$$


Here we used that $\Omega_{\boldsymbol{\varphi}_{\zeta}(c)}=\Omega_{\boldsymbol{\varphi}_{v(c), \mu}(c)}$ and $\Omega^{(c)}:=\Omega_{\boldsymbol{\varphi}_{v}(c), \mu}(c)$. To apply the contraction theorem [6, Thm. 10.1.1] to $g(\boldsymbol{\psi}, \zeta)=\zeta$, it suffices to show that, with $\boldsymbol{\psi} \in b_{R_{1}}$ and $\zeta^{(1)}, \zeta^{(2)} \in B_{\rho_{1}}^{8}$ that a)

$$
\left|g\left(\boldsymbol{\psi}, \zeta^{(1)}\right)-g\left(\boldsymbol{\psi}, \zeta^{(2)}\right)\right| \leq \frac{1}{2}\left|\zeta^{(1)}-\zeta^{(2)}\right| .
$$

and $b$ )

$$
\left.\mid g\left(\boldsymbol{\psi}, \zeta^{(c)}\right)-\zeta^{(c)}\right) \mid \leq \frac{\rho_{2}}{2} .
$$

Then existence, uniqueness and continuity of $\varsigma$ are ensured for $\boldsymbol{\psi} \in b_{R_{1}}\left(\boldsymbol{\varphi}_{\zeta^{(c)}}\right)$.

To translate these two constraints into uniform bounds on the radii $R_{2} \leq R_{1}$ and $\rho_{2} \leq \rho_{1}$, we estimate a 'Taylor series remainder' or, equivalently, use a mean value theorem. First assume that $\boldsymbol{\psi} \in b_{R_{2}}\left(\boldsymbol{\varphi}_{\zeta^{(c)}}\right)$ and $\zeta^{(1)}, \zeta^{(2)} \in B_{R_{2}}^{8}\left(\zeta^{(c)}\right)$. The left-hand side of eq. (C.7) with (C.6) inserted is bounded from above by

$$
A_{1}:=\left|\Omega_{\boldsymbol{\varphi}_{v, \mu}}^{-1}\right|_{\infty}\left|G\left(\boldsymbol{\psi}, \zeta^{(1)}\right)-G\left(\boldsymbol{\psi}, \zeta^{(2)}\right)+\Omega^{(c)}\left(\zeta^{(1)}-\zeta^{(2)}\right)\right|,
$$

where $|\cdot|_{\infty}$ was defined above. Let the vector $f$ be defined through its elements

$$
f_{j}\left(\boldsymbol{\psi}, \zeta^{\prime}\right):=G_{j}\left(\boldsymbol{\psi}, \zeta^{\prime}\right)-\partial_{\zeta} G_{j}\left(\boldsymbol{\varphi}_{\zeta^{(c)}}, \zeta^{(c)}\right) \cdot \zeta^{\prime} .
$$

The difference $f\left(\boldsymbol{\psi}, \zeta^{(1)}\right)-f\left(\boldsymbol{\psi}, \zeta^{(2)}\right)$, which up to a constant describes $A_{1}$, is estimated by the mean value theorem [6, Thm 8.6.2], yielding the upper bound on $A_{1}$ as

$$
\begin{aligned}
A_{1} & \leq\left|\zeta^{(1)}-\zeta^{(2)}\right|\left|\Omega_{\boldsymbol{\varphi}_{v, \mu}}^{-1}\right|_{\infty} \sup _{\zeta \in b_{R_{2}}, \boldsymbol{\psi} \in V_{\rho_{2}}}\left|\Omega^{(c)}-\Omega_{\boldsymbol{\varphi}_{\zeta}}+\left(\boldsymbol{\psi}-\boldsymbol{\varphi}_{\zeta}, J^{-1} \partial_{\zeta, \boldsymbol{z}, \zeta}\right)_{2}\right| \\
& \leq\left|\Omega_{\boldsymbol{\varphi}_{v, \mu}}^{-1}\right|_{\infty}\left(\rho_{2}\left|\Omega_{\boldsymbol{\varphi}_{v, \mu}}\right|_{1, \infty}+R_{2}\left\|\boldsymbol{z}_{\zeta}\right\|_{1, \infty, w}\right)\left|\zeta^{(1)}-\zeta^{(2)}\right| .
\end{aligned}
$$

Here $|\cdot|_{1, \infty}$ is defined above, and to define $\left\|\boldsymbol{z}_{\zeta}\right\|_{1, \infty, w}$, let the matrix $Y$ by its elements $Y_{j k}:=\left\|\partial_{\zeta_{k}} \boldsymbol{z}_{j, \zeta}\right\|_{\tilde{\Gamma}}$, and let the vector $V$ be the elements $V_{k}=\left\|\boldsymbol{z}_{k, \zeta}\right\|_{\tilde{\Gamma}}$. We then have

$$
\left\|\boldsymbol{z}_{\zeta}\right\|_{\infty, w}:=|V|_{\infty}
$$

and

$$
\left\|\boldsymbol{z}_{\zeta}\right\|_{1, \infty, w}:=|Y|_{\infty}+\left\|\boldsymbol{z}_{\zeta}\right\|_{\infty, w} .
$$

Constants, norms, in (C.11), involving $\Omega_{\varphi_{v, \mu}}$ and its inverse are independent of $\zeta$ due to the supremum over $\mu$ and $v$. The numbers $\left\|\partial_{\zeta_{k}} \boldsymbol{z}_{j, \zeta}\right\|_{\tilde{\Gamma}}$ are independent of translation and phase, since the integral over space and its absolute value removes all appearances of phase and translation. Hence $\left\|\boldsymbol{z}_{\zeta}\right\|_{1, \infty, w}$ is independent of $\zeta$ and depends only on $r_{2}^{\prime}$, $I_{2}^{\prime}$. Thus the allowed radii $\rho_{2}$ and $R_{2}$ in (C.11), so that we obtain the constant a half as required in (C.7), only depend on $r_{2}^{\prime}$ and $I_{2}^{\prime}$. We say that (C.7) is uniformly satisfied on $\mathrm{Z}_{2}^{\prime}$.

Similarly, consider (C.8) with (C.6) inserted, its left-hand side is

$$
\left|\left(\Omega^{(c)}\right)^{-1} G\left(\boldsymbol{\psi}, \zeta^{(c)}\right)\right| .
$$


For $\boldsymbol{\psi} \in b_{R_{2}}$, we find

$$
\left|g\left(\boldsymbol{\psi}, \zeta^{(c)}\right)-\zeta^{(c)}\right| \leq\left|\Omega_{\boldsymbol{\varphi}_{v, \mu}}^{-1}\right|_{\infty}\left\|\boldsymbol{z}_{\mu, v}\right\|_{\infty, w} R_{2} \leq \frac{\rho_{2}}{2}
$$

for $R_{2}$ small enough. Thus (C.8) is also uniformly satisfied on $\mathrm{Z}_{2}^{\prime}$ for $R_{2}$ small enough.

The third constraint on the radii $R_{2}$ and $\rho_{2}$ are the conditions to ensure differentiability of $\varsigma$ in $b_{R_{2}}$. To find these constraints we use the differentiability of $G$ and the chain rule. To this end, we consider the Taylor expansion of $G(\boldsymbol{\psi}+\boldsymbol{\xi}, \varsigma(\boldsymbol{\psi}+\boldsymbol{\xi}))$ around the solution $(\boldsymbol{\psi}, \varsigma(\boldsymbol{\psi}))$ :

$$
\begin{aligned}
A_{2}:=\mid G(\boldsymbol{\psi}+\boldsymbol{\xi}, \varsigma(\boldsymbol{\psi}+\boldsymbol{\xi}))-G(\boldsymbol{\psi}, \varsigma(\boldsymbol{\psi})) & -\left(\boldsymbol{\xi}, J \boldsymbol{z}_{,, \varsigma(\boldsymbol{\psi})}\right)_{2} \\
& -\partial_{\zeta .} G(\boldsymbol{\psi}, \varsigma(\boldsymbol{\psi})) \cdot(\varsigma(\boldsymbol{\psi}+\boldsymbol{\xi})-\varsigma(\boldsymbol{\psi})) \mid,
\end{aligned}
$$

which by the construction of $\varsigma$ for $\boldsymbol{\psi}+\boldsymbol{\xi} \in b_{R_{2}}$ reduces to

$$
A_{2}=\left|\left(\boldsymbol{\xi}, J \boldsymbol{z}_{,, \varsigma(\boldsymbol{\psi})}\right)_{2}+\partial_{\zeta} G(\boldsymbol{\psi}, \varsigma(\boldsymbol{\psi})) \cdot(\varsigma(\boldsymbol{\psi}+\boldsymbol{\xi})-\varsigma(\boldsymbol{\psi}))\right|
$$

Differentiability of $G$ ensures a relation between the upper bound of $A_{2}$ and the radii. Let $\tau:=\varsigma(\boldsymbol{\psi}+\boldsymbol{\xi})-\varsigma(\boldsymbol{\psi})$, and let

$$
f_{j}^{1}\left(\boldsymbol{\xi}, \tau^{\prime}\right):=G_{j}\left(\boldsymbol{\psi}+\boldsymbol{\xi}, \varsigma(\boldsymbol{\psi})+\tau^{\prime}\right)-\left(\boldsymbol{\xi}, J \boldsymbol{z}_{j, \zeta}\right)_{2}-\partial_{\varsigma \cdot(\boldsymbol{\psi})} G_{j}(\boldsymbol{\psi}, \varsigma(\boldsymbol{\psi})) \cdot \tau^{\prime}
$$

$A_{2}$ is simply $\left|f^{1}(\boldsymbol{\xi}, \tau)-f^{1}(0,0)\right|$ and by the mean value theorem for we have

$$
\begin{aligned}
& A_{2} \leq|\tau|\left(\left|\Omega_{\boldsymbol{\varphi}_{\varsigma(\psi)+\tau}}-\Omega_{\boldsymbol{\varphi}_{\varsigma(\psi)}}\right|+\left|\left(\boldsymbol{\psi}+\boldsymbol{\xi}-\boldsymbol{\varphi}_{\varsigma(\boldsymbol{\psi})+\tau}, J \partial_{\zeta .} \boldsymbol{z}_{\cdot, \zeta(\boldsymbol{\psi})+\tau}\right)_{2}-\left(\boldsymbol{\psi}-\boldsymbol{\varphi}_{\varsigma(\boldsymbol{\psi})}, J \partial_{\zeta .} \boldsymbol{z}_{\cdot, \zeta(\boldsymbol{\psi})}\right)_{2}\right|\right) \\
& +\|\boldsymbol{\xi}\|_{\tilde{\Gamma}}\left|\boldsymbol{z}_{\varsigma(\boldsymbol{\psi})+\tau}-\boldsymbol{z}_{\varsigma(\boldsymbol{\psi})}\right| \leq|\tau|\left(2\|\boldsymbol{z}\|_{1, \infty, w} R_{2}+\left|\Omega_{\boldsymbol{\varphi}_{v, \mu}}\right|_{1, \infty} \rho_{2}\right)+\|\boldsymbol{\xi}\|_{\tilde{\Gamma}}\left\|\boldsymbol{z}_{\zeta}\right\|_{1, \infty, w} \rho_{2},
\end{aligned}
$$

where we used that $|\tau| \leq \rho_{2}$. The differentiability of $G$ can now be expressed as follows: For every $K_{2}>0$, there exist, by (C.19), radii $R_{2}>0$ and $\rho_{2}>0$, such that

$$
A_{2} \leq K_{2}\left(|\tau|+\|\boldsymbol{\xi}\|_{\tilde{\Gamma}}\right)
$$

The explicit calculation (C.19) shows that (C.20) can be satisfied uniformly on $\mathrm{Z}_{2}^{\prime}$.

To convert the differentiability of $G$ into differentiability of $\varsigma$, we use that $\partial_{\zeta} G$ is invertible at $\boldsymbol{\varphi}_{\zeta^{(c)}}, \zeta^{(c)}$. We recall that

$$
\partial_{\zeta_{k}} G(\boldsymbol{\psi}, \zeta)=-\Omega_{\boldsymbol{\varphi}_{v, \mu}}+\omega\left(\boldsymbol{\psi}-\boldsymbol{\varphi}_{\zeta}, J \partial_{\zeta_{k}} \boldsymbol{z}_{j, \zeta}\right)
$$

For all $\zeta^{\prime} \neq 0$ we have

$$
\left|\partial_{\zeta} G(\boldsymbol{\psi}, \varsigma(\boldsymbol{\psi})) \cdot \zeta^{\prime}\right| \geq\left|\Omega_{\boldsymbol{\varphi}_{v, \mu}} \zeta^{\prime}\right|-\left\|\boldsymbol{z}_{\zeta}\right\|_{1, \infty, w} R_{2}\left|\zeta^{\prime}\right|
$$

and since $\Omega_{\varphi_{v, \mu}}$ is invertible we have

$$
\left|\partial_{\zeta} G(\boldsymbol{\psi}, \varsigma(\boldsymbol{\psi})) \cdot \zeta^{\prime}\right| \geq\left(\left|\Omega_{\boldsymbol{\varphi}_{v, \mu}}^{-1}\right|_{\infty}^{-1}-\left\|\boldsymbol{z}_{\zeta}\right\|_{1, \infty, w} R_{2}\right)\left|\zeta^{\prime}\right| \geq \frac{1}{2}\left|\zeta^{\prime}\right|\left|\Omega_{\boldsymbol{\varphi}_{v, \mu}}\right|_{\infty}>0
$$


by choice of $R_{2}$. For such $R_{2}$ is $\partial_{\zeta} G$ uniformly invertible in each ball $b_{R_{2}}$. If we possibly reduce $\rho_{2}$ and $R_{2}$ further, we may assume that

$$
K_{2}\left|\partial_{\zeta} G(\boldsymbol{\psi}, \varsigma(\boldsymbol{\psi}))^{-1}\right|_{\infty} \leq \frac{1}{2}
$$

and hence by (C.17) and (C.19) we then have

$$
\left|\partial_{\zeta} G^{-1}(\boldsymbol{\psi}, \varsigma(\boldsymbol{\psi}))\left(J \boldsymbol{z}_{\cdot, \zeta}, \boldsymbol{\xi}\right)_{2}+\tau\right| \leq \frac{1}{2}\left(|\tau|+\|\boldsymbol{\xi}\|_{\tilde{\Gamma}}\right)
$$

The triangle inequality leads to

$$
|\tau| \leq\left(1+2\left|\partial_{\zeta} G^{-1}(\boldsymbol{\psi}, \varsigma(\boldsymbol{\psi}))\right|_{\infty}\left\|\boldsymbol{z}_{\zeta}\right\|_{\infty, w}\right)\|\boldsymbol{\xi}\|_{\tilde{\Gamma}} .
$$

Insert this inequality into (C.25) to obtain

$$
\begin{aligned}
\left|\tau+D_{2} G^{-1}(\boldsymbol{\psi}, \varsigma(\boldsymbol{\psi}))\left(D_{1} G(\boldsymbol{\psi}, \varsigma(\boldsymbol{\psi})), \boldsymbol{\xi}\right)_{2}\right| & \\
& \leq 2 K_{2}\left(1+\left|D_{2} G^{-1}(\boldsymbol{\psi}, \varsigma(\boldsymbol{\psi}))\right|_{\infty}\left\|\boldsymbol{z}_{\zeta}\right\|_{\infty, w}\right)\|\boldsymbol{\xi}\|_{\tilde{\Gamma}}
\end{aligned}
$$

and thus $\varsigma$ is differentiable. Furthermore, we have shown that the above constraints can be chosen uniformly in $\zeta$ on $Z_{2}^{\prime}$.

If we now go back and study the restrictions on the radii (C.4), (C.11), (C.15), (C.19), (C.23) and (C.24), we notice that the constraint area can, for a sufficiently small number $\tilde{\rho}_{2}$ such that $\rho_{2} \leq \tilde{\rho}_{2}$, be chosen to be all points in the triangle $0<R_{2} \leq c \rho_{2}, \rho_{2} \leq \tilde{\rho}_{2}$, which concludes the proof of the corollary.

\section{References}

[1] V. S. Buslaev and G. S. Perel'man. Scattering for the nonlinear Schrödinger equation: states that are close to a soliton. Algebra $i$ Analiz, 4(6):63-102, 1992, MR1199635².

[2] V. S. Buslaev and C. Sulem. On asymptotic stability of solitary waves for nonlinear Schrödinger equations. Ann. IHP. Anal. Nonl., 20:419-475, 2003, doi: 10.1016/S0294-1449(02)00018-5 ${ }^{4}$.

[3] S. I. Dejak and B. L. G. Jonsson. Long-time dynamics of variable coefficient mKdV solitary waves. Accepted by J. Math. Phys., 2006, ArXiv:math-ph/0503016 ${ }^{5}$.

[4] S. I. Dejak and I. M. Sigal. Long-time dynamics of KdV solitary waves over a variable bottom. Comm. Pure Appl. Math., 59(6):869-905, 2006, doi: 10.1002/cpa.20120.

\footnotetext{
${ }^{3}$ http://www.ams.org/mathscinet-getitem? $\mathrm{mr}=\mathrm{MR} 1199635$

${ }^{4}$ http://dx.doi.org/10.1016/S0294-1449(02)00018-5

${ }^{5}$ http://arxiv.org/abs/math-ph/0503016
} 
[5] L. Demanet and W. Schlag. Numerical verification of a gap condition for linearized NLS. Nonlinearity, 19(4):829-852, 2006, doi:10.1088/0951-7715/19/4/004.

[6] J. Dieudonné. Foundations of Modern Analysis, volume 10-I of Pure and Applied Mathematics. Academic Press, New York, third edition, 1969, MR0349288. Enlarged and corrected printing.

[7] A. Elgart and B. Schlein. Mean field dynamics of boson stars. Comm. Pure Appl. Math., 2006, doi: 10.1002/cpa.20134. Published online.

[8] J. Fröhlich, S. Gustafson, B. L. G. Jonsson, and I. M. Sigal. Solitary wave dynamics in an external potential. Comm. Math. Phys., 250(3):613-642, 2004, doi: $10.1007 / \mathrm{s} 00220-004-1128-1$.

[9] J. Fröhlich, B. L. G. Jonsson, and E. Lenzmann. Boson stars as solitary waves. Submitted, 2005, ArXiv:math-ph/0512040.

[10] J. Fröhlich, B. L. G. Jonsson, and E. Lenzmann. The kernel condition for boson stars. In preparation, 2006.

[11] J. Fröhlich and E. Lenzmann. Mean-field limit of quantum Bose gases and nonlinear Hartree equation. In Sémin. Équ. Dériv. Partielles. 2003-2004, pages XIX-1-26. École Polytech., Palaiseau, 2004, ArXiv:math-ph/0409019.

[12] J. Fröhlich and E. Lenzmann. Blow-up for nonlinear wave equations describing boson stars. To appear in Comm. Pure Appl. Math., 2005, ArXiv:math.AP/0511003.

[13] J. Fröhlich, T.-P. Tsai, and H.-T. Yau. On the point-particle (Newtonian) limit of the non-linear Hartree equation. Comm. Math. Phys., 225(2):223-274, 2002, doi:10.1007/s002200100579.

[14] I. W. Herbst. Spectral theory of the operator $\left(p^{2}+m^{2}\right)^{1 / 2}-z e^{2} / r$. Comm. Math. Phys., 53(3):285-294, 1977, doi:10.1007/BF01609852.

[15] P. D. Hislop. Exponential decay of two-body eigenfunctions: a review. In Proceedings of the Symposium on Mathematical Physics and Quantum Field Theory (Berkeley, CA, 1999), volume 4 of Electron. J. Differ. Equ. Conf., pages 265-288 (electronic), San Marcos, TX, 2000. Southwest Texas State Univ., MR1785381.

[16] B. L. G. Jonsson, J. Fröhlich, S. Gustafson, and I. M. Sigal. Long time motion of NLS solitary waves in a confining potential. To appear in Ann. Henri Poincare, 2006, ArXiv:math-ph/0503009.

[17] T. Kato. Perturbation theory for linear operators. Classics in Mathematics. SpringerVerlag, Berlin, 1995, MR1335452. Reprint of the 1980 edition.

[18] D. J. Kaup. A perturbation expansion for the Zakharov-Shabat inverse scattering transform. SIAM J. Appl. Math., 31(1):121-133, 1976, doi:10.1137/0131013. 
[19] Y. Kodama and M. J. Ablowitz. Perturbations of solitons and solitary waves. Stud. Appl. Math., 64(3):225-245, 1981, MR615541.

[20] E. Lenzmann. Well-posedness for semi-relativistic Hartree equations of critical type. To appear in Math. Phys. Anal. Geom., 2006, ArXiv:math.AP/0505456.

[21] E. H. Lieb and M. Loss. Analysis, volume 14 of Graduate Studies in Mathematics. American Mathematical Society, Providence, RI, second edition, 2001, MR1817225.

[22] E. H. Lieb and W. E. Thirring. Gravitational collapse in quantum mechanics with relativistic kinetic energy. Ann. Physics, 155(2):494-512, 1984, doi:10.1016/00034916(84)90010-1.

[23] E. H. Lieb and H.-T. Yau. The Chandrasekhar theory of stellar collapse as the limit of quantum mechanics. Comm. Math. Phys, 112(1):147-174, 1987, doi:10.1007/BF01217684.

[24] D. W. McLaughlin and A. C. Scott. Perturbation analysis of fluxon dynamics. Phys. Rev. A, 18(4):1652-1680, 1978, doi:10.1103/PhysRevA.18.1652.

[25] F. Merle and P. Raphael. The blow-up dynamic and upper bound on the blow-up rate for critical nonlinear Schrödinger equation. Ann. of Math. (2), 161(1):157-222, 2005, euclid.annm/1111509197 ${ }^{6}$.

[26] J. Messer. Temperature dependent Thomas-Fermi theory, volume 147 of Lect. Notes Phys. Springer, Berlin, 1981, doi:10.1007/3-540-10875-0.

[27] R. Ruffini and S. Bonazzola. Systems of self-graviting particles in general relativity and the concept of an equation of state. Phys. Rev. II, 187(5):1767-1783, 1969, doi:10.1103/PhysRev.187.1767.

[28] E. L. Slaggie and E. H. Wichmann. Asymptotic properties of the wave function for a bound nonrelativistic three-body system. J. Math. Phys., 3:946-968, 1962, doi: $10.1063 / 1.1724311$.

[29] E. M. Stein. Harmonic analysis: real-variable methods, orthogonality, and oscillatory integrals, volume 43 of Princeton Mathematical Series. Princeton University Press, 1993, MR 1232192. With the assistance of Timothy S. Murphy, Monographs in Harmonic Analysis, III.

[30] D. M. A. Stuart. Perturbation theory for kinks. Comm. Math. Phys., 149(3):433462, 1992, doi:10.1007/BF02096938.

[31] D. M. A. Stuart. Modulational approach to stability of non-topological solitons in semilinear wave equations. J. Math. Pure. Appl., 80(1):51-83, 2001, doi: 10.1016/S0021-7824(00)01189-2.

\footnotetext{
${ }^{6}$ http://projecteuclid.org/getRecord?id=euclid.annm/1111509197
} 
[32] D. M. A. Stuart. Geodesics and the Einstein nonlinear wave system. J. Math. Pure. Appl., 83(5):541-587, 2004, doi:10.1016/j.matpur.2003.09.009.

[33] W. Thirring. Bosonic black holes. Phys. Lett. B, 127(1-2):27-29, 1983, doi: 10.1016/0370-2693(83)91623-4.

[34] R. Weder. Spectral properties of one-body relativistic spin-zero Hamiltonians. Ann. Inst. H. Poincaré Sect. A (N.S.), 20:211-220, 1974.

[35] M. I. Weinstein. Modulational stability of ground states of nonlinear Schrödinger equations. SIAM J. Math. Anal., 16(3):472-491, 1985, doi:10.1137/0516034.

[36] E. Zeidler. Applied Functional Analysis, Main Principles and Their Applications, volume 109 of Applied Mathematical Sciences. Springer-Verlag, New York, 1995, MR1347692.

JÜRG FRÖHLICH

Institute For Theoretical Physics

ETH ZÜRICH

CH-8093 ZÜRICH, Switzerland.

email: juerg@itp.phys.ethz.ch

B. LARS G. JONSSON

Institute for Theoretical Physics

ETH ZüRICH

CH-8093 ZÜriCh, Switzerland.

Alternative address:

School of Electrical EngineERING

EleCtromagnetic ENGINEERING

Royal Institute of Technology (KTH)

SE-100 44 Stockholm.

email: jonsson@itp.phys.ethz.ch

ENNO LENZMANN

Department of Mathematics, HG G 33.1

ETH ZÜRICH

CH-8092 Zürich, Switzerland.

email: lenzmann@math.ethz.ch 